\title{
Financial Services Used by Small Businesses: Evidence from the 2003 Survey of Small Business Finances
}

Traci L. Mach and John D. Wolken, of the Board's Division of Research and Statistics, prepared this article. Courtney M. Carter, John A. Holmes, and Lieu N. Hazelwood provided research assistance.

Small businesses-nonfarm entities with fewer than 500 employees-are an integral part of the U.S. economy. They account for about half of privatesector output, employ more than half of private-sector workers, and have generated 60 percent to 80 percent of net new jobs annually over the past decade. ${ }^{1}$ Given the significant role of small businesses in the national economy, understanding trends in the types and sources of financing they use is important for economic research and policymaking, especially because small businesses typically finance their operations quite differently than large corporations do. For example, a small business often relies on the personal resources and credit history of the firm's owners to access credit.

Newly available data from the Federal Reserve Board's 2003 Survey of Small Business Finances (SSBF) provide detailed information on the use of credit and other financial services by these firms. The SSBF is the most comprehensive source of information available on the characteristics of small businesses and their owners; no other survey provides the breadth and detail of information for a nationally representative sample of such firms. Moreover, policymakers and researchers can compare the newest SSBF data with results from the previous surveys, which cover 1987, 1993, and 1998. Most of the

\footnotetext{
1. These proportions are relative to the nonfarm sector and are from "Frequently Asked Questions" at the website of the U.S. Small Business Administration (SBA), Office of Advocacy, http:// app1.sba.gov/faqs/faqIndexAll.cfm?areaid=24. For research purposes, the SBA Office of Advocacy defines a small business as an independent firm having fewer than 500 employees. For purposes of contracting with the federal government, small businesses are defined by the SBA Office of Size Standards, and the definition varies by industry and in some cases is by dollar value of sales or of assets rather than number of employees. This article will in some instances also use sales or assets to define subsets of small businesses.
}

changes reported in this article are for the period between the 1998 and 2003 surveys. $^{2}$

The latest survey gathered data from 4,240 firms selected to be representative of small businesses operating in the United States at the end of $2003 .{ }^{3}$ As in previous surveys, the data show that most businesses were very small and were located in urban areas. Also as in previous surveys, the percentage of firms involved in the provision of business and professional services increased somewhat, whereas the percentages of firms engaged in manufacturing and in retail and wholesale trade declined. Among firms that were corporations, those organized under subchapter $\mathrm{S}$ of the U.S. Internal Revenue Code (S corporations) continued to grow as a proportion of all small businesses relative to those organized under subchapter $\mathrm{C}$ (C corporations). ${ }^{4}$

2. The 1987 and 1993 surveys were called the National Survey of Small Business Finances. For summaries of the earlier surveys, refer to the following articles: Gregory E. Elliehausen and John D. Wolken (1990), "Banking Markets and the Use of Financial Services by Small and Medium-Sized Businesses," Federal Reserve Bulletin, vol. 76 (October), pp. 801-17; Rebel A. Cole and John D. Wolken (1995), "Financial Services Used by Small Businesses: Evidence from the 1993 National Survey of Small Business Finances," Federal Reserve Bulletin, vol. 81 (July), pp. 629-67; and Marianne P. Bitler, Alicia M. Robb, and John D. Wolken (2001), "Financial Services Used by Small Businesses: Evidence from the 1998 Survey of Small Business Finances," Federal Reserve Bulletin, vol. 87 (April), pp. 183-205. Information on the availability of the SSBF data as well as technical information, data from previous surveys, and a bibliography of research using the SSBF are available on the website of the Federal Reserve Board, at www.federalreserve.gov/pubs/oss/oss3/nssbftoc.htm.

3. Interviewing began in mid-2004 and for the most part was completed by year-end. Firms were asked to report balance sheet and income data for the firm's fiscal year that ended between May 1, 2003, and April 30, 2004; other data were reported as of the date of the interview. Results from the 2003 survey are referred to in this article as 2003 data. Further information on the survey's methodology is in appendix A.

4. The organizational forms have different rules about liability and taxes. In sole proprietorships (hereafter, proprietorships) the owners receive all the income from the business and bear full liability for its obligations. Partnerships must have more than one owner. As in proprietorships, the partners receive all the income from the business and, in general, are fully liable for its obligations. Corporations are separate legal entities, and the owners' liability is limited to the amount of their original equity investment. The primary difference 
The financial affairs of small business in 2003 were conducted in a financial marketplace whose elements-including regulations, technology, and organizational structures-have changed markedly since the Federal Reserve Board's first small business survey. For example, state and federal restrictions on interstate branching and banking have been relaxed, certain financial institutions are now permitted to offer a wider range of financial services, lenders employ complex credit-scoring models to evaluate would-be borrowers, and mergers and acquisitions have produced a financial industry with fewer but larger organizations.

In this changing financial marketplace, small businesses have been diversifying their providers of financial services. Nondepository institutions have become increasingly important sources of financial services to small businesses; more than half reported using nondepository sources in 2003, compared with about 40 percent in 1998. Among these sources, finance companies and leasing companies were important suppliers of credit and financial management services, especially for the largest small businesses, and brokerage firms were important suppliers of brokerage and trust and pension services. Nonetheless, commercial banks continued to be, by a wide margin, the supplier most commonly used by small businesses for checking and savings accounts, for loans other than leases and vehicle loans, and for financial management services other than brokerage and trust and pension services. They were the second most commonly reported provider of vehicle loans and trust and pension services.

The types of credit used by small businesses have also been changing. The percentage of firms that had outstanding vehicle loans and credit lines increased between the 1998 and 2003 surveys; the use of capital leases declined somewhat; and the use of equipment loans, mortgages, and other loans remained about the same. The use of personal credit cards for business purposes remained roughly constant, whereas the use of business credit cards increased substantially.

This article focuses on some of the major results from the 2003 SSBF for broad subgroups of small businesses. ${ }^{5}$ Understanding and explaining many of

between the two types of corporations is how they are taxed: $\mathrm{S}$ corporations are not subject to corporate income tax, whereas C corporations are. $\mathrm{S}$ corporations are legally constrained to have fewer than seventy-five shareholders, are restricted to one class of stock, and must pass all firm income to the owners at the end of each fiscal year.

5. Space limitations prevent the inclusion of standard errors for all the statistics presented here. Although we do not directly address the statistical significance of the results, the article highlights findings that are significant or are interesting in a broader context. the findings may require a more-detailed and in-depth analysis than is possible in this article. To facilitate additional research, a micro-level data set for public use will be released shortly after the publication of this article. ${ }^{6}$ These data will permit a rigorous analysis that takes into account characteristics of the businesses, their owners, and local banking markets. Researchers will be able to study many aspects of small business finance, including, for example, how the proximity of financial institutions affects the mix of financial products the firm uses, which characteristics of firms and owners affect the ability of small businesses to obtain credit, and how lending patterns vary with these characteristics.

\section{ECONOMIC AND FINANCIAL SERVICES ENVIRONMENT}

In 1998 the economy was in its seventh year of sustained economic expansion. The annual unemployment rate had fallen to 4.5 percent; the consumer price index rose 1.6 percent, gross domestic product grew 4.4 percent, and productivity in the nonfarm business sector increased 2.7 percent.

In 2003 the economic climate for small businesses was quite different than in 1998. A recession in 2001 was followed by a sluggish recovery. By the end of 2003, the pace of economic activity was picking up, although many small businesses were likely still feeling some effects from the subpar performance in the preceding few years. Many small businesses had failed, and those that had weathered the period were probably facing declining revenues. Health-care costs had increased sharply, venture capital opportunities had declined, and banks had instituted new fees and raised existing fees and balance requirements. At the same time, interest rates in 2003 were lower than they had been in decades; these low rates made relatively low cost new loans available and provided opportunities for substantial savings from refinancing.

These differences in the overall economy between the two most recent surveys are reflected in the problems reported by firms (table 1). In 2003, poor sales topped the list, particularly among the smallest firms. In 1998, firms reported that their most important problems were competition from other firms and the quality of labor. The quality of labor remained a commonly reported concern in 2003 , especially among firms with ten or more employees. The 2003 survey also recorded a marked increase in the percentage of

6. The data will be available on the Federal Reserve Board's website, at www.federalreserve.gov/pubs/oss/oss3/nssbftoc.htm 
1. Most important problem reported by small businesses, by number of employees in firm, 1998 and 2003 surveys Percent

\begin{tabular}{|c|c|c|c|c|c|c|c|c|}
\hline \multirow{2}{*}{ Problem } & \multicolumn{4}{|c|}{1998} & \multicolumn{4}{|c|}{2003} \\
\hline & 9 or fewer & $10-49$ & $50-99$ & $100-499$ & 9 or fewer & $10-49$ & $50-99$ & $100-499$ \\
\hline Taxes ... & 7.2 & 5.5 & 2.8 & 4.0 & 5.4 & 6.1 & 1.5 & 2.9 \\
\hline Inflation . . & .4 & .4 & .3 & .3 & 1.9 & 3.7 & 2.9 & 2.2 \\
\hline Poor sales $\ldots \ldots \ldots \ldots \ldots \ldots$. & 7.6 & 6.5 & 7.6 & 7.1 & 20.9 & 13.7 & 15.4 & 12.3 \\
\hline Financing and interest rates & 6.9 & 6.7 & 4.2 & 3.2 & 6.5 & 3.5 & 3.0 & 1.5 \\
\hline Cost of labor $\ldots \ldots \ldots \ldots \ldots \ldots \ldots$ & 3.2 & 6.2 & 14.8 & 9.7 & 1.2 & 1.9 & 4.1 & 2.6 \\
\hline Government regulations and red tape . & 6.9 & 6.0 & 7.4 & 8.9 & 4.1 & 4.9 & 8.2 & 5.0 \\
\hline Competition from larger firms...$\ldots \ldots$ & 11.0 & 12.6 & 9.7 & 15.6 & 3.3 & 4.5 & 5.1 & 4.0 \\
\hline Quality of labor .................... & 10.2 & 23.7 & 25.6 & 24.4 & 4.8 & 15.3 & 13.8 & 15.1 \\
\hline Cost and availability of insurance .... & 2.3 & 2.5 & .3 & .1 & 8.5 & 14.9 & 13.7 & 14.6 \\
\hline Other $\ldots \ldots \ldots \ldots \ldots \ldots \ldots \ldots \ldots \ldots \ldots \ldots \ldots \ldots$ & 44.3 & 29.9 & 27.4 & 26.7 & 43.4 & 31.5 & 32.3 & 39.8 \\
\hline Total & 100 & 100 & 100 & 100 & 100 & 100 & 100 & 100 \\
\hline
\end{tabular}

NoTE: In this article, number of employees consists of full- and part-time workers, counted equally, and owners who work in the firm; it excludes workers employed temporarily or under other nonstandard work arrangements (refer to table 5). Here and in subsequent tables except as noted, percentages are weighted to adjust for differences in sampling and response rates; the weighted

firms reporting the cost and availability of insurance as their most important problem.

\section{CHARACTERISTICS OF SMALL BUSINESSES}

Like its predecessors, the 2003 SSBF collected a wide variety of information about firms and owners, including the firm's size, primary industry, and organizational structure and the owners' race, ethnicity, sex, and extent of participation in the firm (table 2).

The composition of small businesses has remained largely unchanged between the 1998 and 2003 surveys. The large majority continued to be very small and owner-managed. More than 80 percent of firms employed fewer than ten workers, and less than 3 percent employed fifty or more. ${ }^{7}$ More than 70 percent of firms had annual sales of less than $\$ 500,000$, and more than 80 percent had assets of less than $\$ 500,000$. Finally, more than 85 percent conducted business out of a single location, and the vast majority of owners (94 percent) managed day-to-day activities themselves.

In 2003, 47 percent of all small businesses were corporations (31 percent were $\mathrm{S}$ corporations and 16 percent were $\mathrm{C}$ corporations), 45 percent were proprietorships, and the remaining 9 percent were partnerships. The proportion of S corporations relative to C corporations has grown since 1993, when they accounted for 20 percent and 30 percent, respectively, of all small businesses. A portion of this shift may be attributable to the Small Business Job Protec-

7. The number of employees includes paid and unpaid workers and owners who work in the firm; part-time and full-time workers are each counted as one. For example, if a total of two part-time workers, one full-time worker, and an owner work in the firm, the firm is considered to have four employees. data reflect the population of small businesses rather than sample measures (more information is available in the appendix). Also here and in subsequent tables, components may not sum to totals because of rounding, or because some firms did not answer or answered "Do not know," or, in a few cases, because values for some variables are missing.

tion Act of 1996, which liberalized the rules for subchapter $\mathrm{S}$ qualification. ${ }^{8}$

Service industries (both business and professional services) accounted for the largest fraction-46 percent-of small businesses' primary activities, and 18 percent of all firms were primarily in retail trade. This distribution is similar to that in 1998; between the two surveys, small increases were observed in business and professional services and small decreases in manufacturing and retail and wholesale trade.

The geographic distribution of the firms corresponded closely to the distribution of the population: 35 percent in the South, 24 percent in the West, 21 percent in the Midwest, and 20 percent in the Northeast. ${ }^{9}$ About 79 percent of firms had their headquarters or main office in an urban area, and the remaining 21 percent were in rural areas. The vast majority of the firms (95 percent) conducted business primarily within the United States, and the remaining 5 percent operated internationally.

\section{Number and Ownership Shares of Small Business Owners}

Information on the owners of the firm was collected differently for the 2003 survey than it had been

8. The act increased the number of permitted shareholders from thirty-five to seventy-five; allowed an "electing small business trust" with multiple beneficiaries to qualify as an S corporation shareholder; allowed charitable organizations and qualified retirement plans (but not individual retirement accounts) to be $\mathrm{S}$ corporation shareholders; and allowed corporations with subsidiaries to become $\mathrm{S}$ corporations (and provided that wholly owned subsidiaries could be considered part of the $\mathrm{S}$ corporation for federal income tax purposes).

9. In 2003, 36 percent of the population was in the South, 23 percent in the West, 22 percent in the Midwest and 19 percent in the Northeast. Geographic areas of the United States cited in this article are as defined by the U.S. Census Bureau (www.census.gov/geo/www/ us_regdiv.pdf). 
2. Number and population proportion of small businesses in survey sample, by selected characteristics of firms, 2003 survey

\begin{tabular}{|c|c|c|c|}
\hline Characteristic & $\begin{array}{l}\text { Number } \\
\text { in sample }\end{array}$ & $\begin{array}{c}\text { Percentage } \\
\text { of population }\end{array}$ & $\begin{array}{c}\text { Мемо } \\
1998 \\
\text { percentage of } \\
\text { population }^{2}\end{array}$ \\
\hline All firms $\ldots \ldots \ldots \ldots \ldots \ldots \ldots$ & 4,240 & 100 & 100 \\
\hline 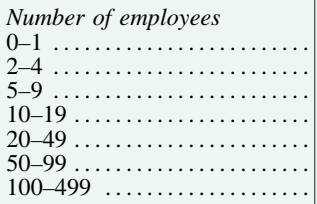 & $\begin{array}{r}640 \\
1,167 \\
632 \\
389 \\
566 \\
444 \\
402\end{array}$ & $\begin{array}{r}20.6 \\
40.0 \\
20.2 \\
10.6 \\
6.0 \\
1.7 \\
1.0\end{array}$ & $\begin{array}{r}21.9 \\
41.5 \\
19.6 \\
8.8 \\
5.6 \\
1.6 \\
1.2\end{array}$ \\
\hline 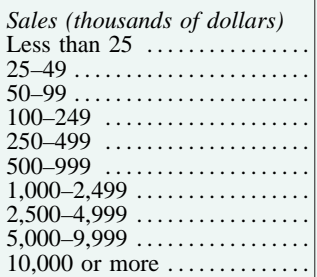 & $\begin{array}{l}430 \\
289 \\
350 \\
598 \\
459 \\
441 \\
532 \\
338 \\
319 \\
483\end{array}$ & $\begin{array}{r}14.6 \\
9.9 \\
11.6 \\
19.8 \\
14.3 \\
12.2 \\
10.0 \\
3.6 \\
2.3 \\
1.7\end{array}$ & $\begin{array}{r}16.3 \\
9.4 \\
14.3 \\
21.9 \\
13.3 \\
10.2 \\
8.1 \\
3.3 \\
1.6 \\
1.7\end{array}$ \\
\hline 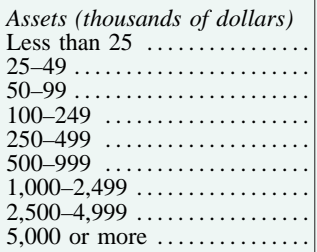 & $\begin{array}{l}934 \\
372 \\
447 \\
573 \\
401 \\
361 \\
439 \\
279 \\
434\end{array}$ & $\begin{array}{r}31.3 \\
12.5 \\
13.5 \\
15.9 \\
10.0 \\
7.1 \\
5.8 \\
1.9 \\
2.0\end{array}$ & $\begin{array}{r}34.8 \\
12.8 \\
14.2 \\
15.7 \\
9.0 \\
6.1 \\
4.3 \\
1.7 \\
1.4\end{array}$ \\
\hline $\begin{array}{l}\text { Organizational form } \\
\text { Proprietorship } \ldots \ldots \ldots \ldots \ldots \\
\text { Partnership } \ldots \ldots \ldots \ldots \ldots \ldots \\
\text { S corporation } \ldots \ldots \ldots \ldots \ldots \\
\text { C corporation } \ldots \ldots \ldots \ldots \ldots \ldots\end{array}$ & $\begin{array}{r}1,347 \\
344 \\
1,548 \\
1,001\end{array}$ & $\begin{array}{r}44.5 \\
8.7 \\
31.0 \\
15.8\end{array}$ & $\begin{array}{r}49.4 \\
7.0 \\
23.9 \\
19.8\end{array}$ \\
\hline $\begin{array}{l}\text { Standard Industrial } \\
\text { Classification } \\
\text { Construction and mining } \ldots \ldots \\
\text { Manufacturing } \ldots \ldots \ldots \ldots \ldots \ldots \\
\text { Transportation } \ldots \ldots \ldots \ldots \ldots \ldots \\
\text { Wholesale trade } \ldots \ldots \ldots \ldots \ldots \\
\text { Retail trade } \ldots \ldots \ldots \ldots \ldots \\
\text { Insurance and real estate } \ldots \ldots \\
\text { Business services } \ldots \ldots \ldots \ldots \\
\text { Professional services } \ldots \ldots \ldots \ldots\end{array}$ & $\begin{array}{l}440 \\
499 \\
171 \\
288 \\
821 \\
262 \\
934 \\
823\end{array}$ & $\begin{array}{r}11.8 \\
7.1 \\
3.8 \\
5.9 \\
18.4 \\
18.4 \\
7.2 \\
25.1 \\
20.7\end{array}$ & $\begin{array}{r}11.9 \\
8.3 \\
3.7 \\
7.2 \\
19.0 \\
6.5 \\
24.8 \\
18.5\end{array}$ \\
\hline 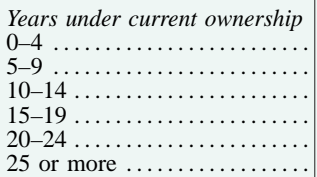 & $\begin{array}{l}686 \\
822 \\
666 \\
596 \\
512 \\
957\end{array}$ & $\begin{array}{l}20.6 \\
22.1 \\
16.0 \\
12.6 \\
10.9 \\
17.9\end{array}$ & $\begin{array}{r}22.4 \\
22.8 \\
19.2 \\
12.9 \\
8.9 \\
14.0\end{array}$ \\
\hline
\end{tabular}

previously. In the past, characteristics of owners were collected only for the owner with the largest share, and respondents were asked whether a majority of firm owners were Hispanic, nonwhite, or female. The 2003 survey followed the lead taken by the U.S. Census Bureau in its Survey of Business Owners and collected demographic information on up to three owners. ${ }^{10}$ Respondents were asked to report first on the individual with the largest ownership share (re-

10. Further information on the Census Bureau survey is available at www.census.gov/sbo/index.html.
2.-Continued

\begin{tabular}{|c|c|c|c|}
\hline Characteristic & $\begin{array}{l}\text { Number } \\
\text { in sample }\end{array}$ & $\begin{array}{c}\text { Percentage } \\
\text { of population }\end{array}$ & $\begin{array}{c}\text { Мемо } \\
1998 \\
\text { percentage of } \\
\text { population }^{2}\end{array}$ \\
\hline \multicolumn{4}{|l|}{ Census area of main office } \\
\hline Northeast $\ldots \ldots \ldots \ldots \ldots$ & 756 & 19.8 & 18.9 \\
\hline New England & 247 & 6.0 & 5.2 \\
\hline Middle Atlantic & 509 & 13.8 & 13.7 \\
\hline \multirow{3}{*}{ 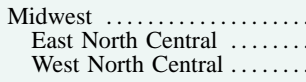 } & 1,015 & 21.1 & 21.8 \\
\hline & 652 & 14.2 & 14.6 \\
\hline & 363 & 6.9 & 7.2 \\
\hline South .......... & 1,386 & 34.7 & 32.7 \\
\hline South Atlantic ... & 747 & 18.9 & 16.9 \\
\hline \multirow{2}{*}{$\begin{array}{l}\text { East South Central } \\
\text { West South Central }\end{array}$} & 231 & 5.3 & 5.5 \\
\hline & 408 & 10.5 & 10.4 \\
\hline West ........ & 1,083 & 24.4 & 26.6 \\
\hline Mountain & 344 & 7.6 & 6.6 \\
\hline Pacific .... & 739 & 16.8 & 20.0 \\
\hline \multicolumn{4}{|l|}{ Urbanization at main office } \\
\hline \multirow{2}{*}{$\begin{array}{l}\text { Urban } \ldots \ldots \ldots \ldots \ldots \ldots \ldots \ldots \ldots \ldots \ldots \ldots \ldots \\
\text { Rural } \ldots \ldots \ldots \ldots \ldots \ldots \ldots\end{array}$} & 3,350 & 79.4 & 79.9 \\
\hline & 890 & 20.6 & 20.1 \\
\hline \multicolumn{4}{|l|}{ Number of offices } \\
\hline \multirow{3}{*}{ 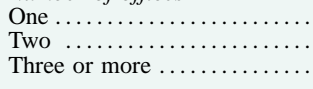 } & 3,235 & 86.0 & 87.8 \\
\hline & 474 & 9.4 & 8.6 \\
\hline & 531 & 4.6 & 3.6 \\
\hline \multicolumn{4}{|l|}{ Sales area } \\
\hline \multicolumn{4}{|l|}{ Primarily within the } \\
\hline $\begin{array}{l}\text { United States } \ldots \ldots \\
{\text { International or } \text { global }^{3}}^{3}\end{array}$ & $\begin{array}{r}3,995 \\
245\end{array}$ & $\begin{array}{r}95.4 \\
4.6\end{array}$ & $\begin{array}{r}95.5 \\
4.5\end{array}$ \\
\hline \multicolumn{4}{|l|}{ Management } \\
\hline \multirow{2}{*}{$\begin{array}{l}\text { By owner } \ldots \ldots \ldots \ldots \ldots \ldots \\
\text { Hired } \ldots \ldots \ldots \ldots \ldots \ldots \ldots\end{array}$} & 3,794 & 94.3 & 92.5 \\
\hline & 387 & 5.8 & 7.5 \\
\hline \multicolumn{4}{|l|}{$\begin{array}{l}\text { Race, ethnicity, and sex of } \\
\text { majority owners }\end{array}$} \\
\hline \multirow{2}{*}{$\begin{array}{l}\text { Nonwhite or Hispanic } \ldots . \\
\text { Non-Hispanic white } \ldots .\end{array}$} & 484 & 13.1 & 14.6 \\
\hline & 3,697 & 86.6 & 85.4 \\
\hline \multirow{2}{*}{$\begin{array}{l}\text { White } \ldots \ldots \ldots \ldots \ldots \ldots \ldots \\
\text { Black } \ldots \ldots \ldots \ldots \ldots \ldots \ldots\end{array}$} & 3,853 & 91.0 & 90.7 \\
\hline & 119 & 3.7 & 4.1 \\
\hline $\begin{array}{c}\text { Asian, Native Hawaiian, or } \\
\text { other Pacific Islander ... }\end{array}$ & 170 & 4.2 & 4.4 \\
\hline $\begin{array}{c}\text { American Indian or Alaska } \\
\text { Native } \ldots . . . \ldots \ldots \ldots . . .\end{array}$ & 58 & 1.3 & .8 \\
\hline \multirow{2}{*}{$\begin{array}{l}\text { Hispanic ...... } \\
\text { Non-Hispanic }\end{array}$} & 149 & 4.2 & 5.6 \\
\hline & 4,032 & 95.8 & 94.4 \\
\hline \multirow{3}{*}{$\begin{array}{l}\text { Female } \ldots \ldots \ldots \ldots \ldots \ldots \ldots \\
\text { Male } \ldots \ldots \ldots \ldots \ldots \ldots \\
\text { Ownership divided equally } \\
\quad \text { by } \operatorname{sex} \ldots \ldots \ldots \ldots \ldots \ldots\end{array}$} & 783 & 22.4 & 24.3 \\
\hline & 2,923 & 64.8 & 72.0 \\
\hline & 475 & 12.8 & 3.7 \\
\hline
\end{tabular}

1. Unweighted.

2. The percentages reported here are final and may differ slightly from the preliminary data reported in Bitler, Robb, and Wolken (2001), "Financial Services Used by Small Businesses."

3. International refers to sales areas outside the United States; global refers to combined U.S. and international sales areas.

ferred to in this article as the first owner); if that individual did not have a controlling interest in the company (an ownership share of at least 51 percent), information was also collected on up to two additional owners.

This new method confirmed the implicit assumption under which previous information was collected about firm owners: Small businesses are very closely held. The average firm had only three owners, and the owner with the largest share held an 81.5 percent interest in the firm (table 3). The largest differences in ownership dispersion of the firms can be seen across 
3. Number of owners and average ownership shares of principal owners, by number of employees and organizational form of the firm, 2003 survey

\begin{tabular}{|c|c|c|c|c|}
\hline \multirow{2}{*}{ Characteristic } & \multicolumn{2}{|c|}{ Number of owners } & \multicolumn{2}{|c|}{$\begin{array}{l}\text { Ownership share } \\
\text { (percent) }\end{array}$} \\
\hline & Median & Average & $\begin{array}{l}\text { First } \\
\text { owner }\end{array}$ & $\begin{array}{l}\text { Second } \\
\text { owner }\end{array}$ \\
\hline All firms $\ldots \ldots \ldots \ldots \ldots$ & 1 & 3.0 & 81.5 & 44.3 \\
\hline 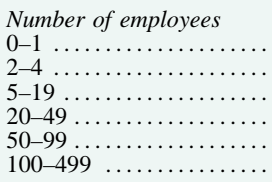 & $\begin{array}{l}1 \\
1 \\
2 \\
2 \\
2 \\
3\end{array}$ & $\begin{array}{r}1.2 \\
1.6 \\
2.4 \\
8.7 \\
43.8 \\
13.0\end{array}$ & $\begin{array}{l}94.6 \\
82.5 \\
75.2 \\
69.7 \\
66.2 \\
62.9\end{array}$ & $\begin{array}{l}48.3 \\
46.5 \\
44.0 \\
38.6 \\
35.9 \\
30.2\end{array}$ \\
\hline $\begin{array}{l}\text { Organizational form } \\
\text { Proprietorship } \ldots \ldots \ldots \ldots \\
\text { Partnership } \ldots \ldots \ldots \ldots \ldots \\
\text { S corporation } \ldots \ldots \ldots \ldots \ldots \\
\text { C corporation } \ldots \ldots \ldots \ldots\end{array}$ & $\begin{array}{l}1 \\
2 \\
2 \\
2\end{array}$ & $\begin{array}{r}1.2 \\
2.9 \\
2.0 \\
10.2\end{array}$ & $\begin{array}{l}93.6 \\
52.3 \\
76.6 \\
73.0\end{array}$ & $\begin{array}{l}50.0 \\
42.9 \\
44.5 \\
41.0\end{array}$ \\
\hline
\end{tabular}

Note: The survey designates the owner with the largest share as the first owner, and the owner with the second-largest share as the second owner.

organizational type and firm size. Among partnerships, the average firm had 2.9 owners, and the partner with the largest share controlled 52.3 percent of the firm. Compared with partnerships, $\mathrm{C}$ corporations had more owners (10.2 on average), but the largest owner held a larger share of the firm (73.0 percent). S corporations had 2 owners on average, with the largest shareholder controlling 76.6 percent of the firm.

The average number of owners increased with the number of employees: The smallest firms $(0-1 \mathrm{em}-$ ployees) had an average of 1.2 owners; intermediatesized firms (5-19 employees and 20-49 employees) had 2.4 and 8.7 owners respectively; and the largest firms (100-499 employees) had 13 owners. The ownership share of the first owner decreased as the number of owners increased, from 94.6 percent among the smallest firms to 62.9 percent among the largest.

\section{Race, Ethnicity, and Sex of Small Business Owners}

The race, ethnicity, and sex of the ownership of a small business in the survey were defined by the weighted sum of the characteristics of the firms' owners. ${ }^{11}$ Unlike in previous years, owners were allowed to identify themselves as being of more than one race, and therefore firms could be classified as being of more than one race. For firms in which less than 100 percent of the ownership was reported, characteristics were scaled up by a factor that made the reported ownership equal 100 percent. If the

11. Characteristics of each owner were weighted by the owner's share in the business. characteristic was 51 percent or more, the firm was determined to be of that group. ${ }^{12}$

In 2003, 13.1 percent of firms were owned by nonwhite or Hispanic individuals (table 2); the share is statistically lower than that recorded by the 1998 survey (14.6 percent). The shares for nonwhite groups alone did not change by a statistically significant amount: The share for blacks and the share for Asians each held at roughly 4 percent; ${ }^{13}$ the share for American Indians and Alaska Natives held at roughly 1 percent. However, the share of Hispanic-owned firms fell a statistically significant amount, from 5.6 percent to 4.2 percent (refer to appendix B for a discussion of changes in the estimated rates of nonwhite and Hispanic ownership).

The largest change in ownership composition in 2003 was among firms owned equally by males and females. The proportion of such firms rose sharply, from 3.7 percent in 1998 to 12.8 percent in 2003, although part of this increase may stem from changes in how the question was asked. ${ }^{14}$ This increase is reflected in the decline in the percentage of firms that were owned by males, from 72.0 percent to 64.8 percent; the percentage of firms owned by females also declined between the two surveys, but much lessfrom 24.3 percent to 22.4 percent.

Firms owned by females, nonwhites, or Hispanics differed in several ways from firms owned by males, whites, or non-Hispanics (table A.1). As seen in

12. For example, consider a firm in which the total reported shares summed to 75 percent. The largest owner held 40 percent of the firm and identified himself as both white and nonblack, as Hispanic, and as male; the second owner held 25 percent of the firm and identified herself as both white and black, as non-Hispanic, and as female; and the third owner held 10 percent of the firm and identified himself as both nonwhite and black, as non-Hispanic, and as male. The ownershipweighted characteristics of the owners were 87 percent white, 46 percent black, 53 percent Hispanic, and 67 percent male. The firm would be deemed to be white, Hispanic, and male (but not black because the ownership-weighted percentage of black ownership was less than 51 percent). Here is how the firm's share of Hispanic ownership would be calculated in this example:

$[(1 \times .40)+(0 \times .25)+(0 \times .10)] \times(1 / .75) \times 100=53$ percent. The first term, $1 \times .40$, is the product of an indicator of the first owner's Hispanic status (1, indicating Hispanic) times the first owner's share (40 percent). The second term is the product of an indicator of the second owner's Hispanic status (0, indicating non-Hispanic) times the second owner's share, and the third term is the product of an indicator of the third owner's Hispanic status times the third owner's share. The sum of the three terms are then multiplied by the reciprocal of the total shares reported $(.40+.25+.10)=.75$ so that the total shares will sum to 100 percent. Last, the adjusted sum is multiplied by 100 to convert the value to percent.

13. Throughout this article, the term "Asian" is used for convenience to refer to individuals who characterized themselves as being in the category "Asian, Native Hawaiian, or other Pacific Islander."

14. This increase is likely to be at least partially attributable to the fact that equal ownership was a "volunteered" response rather than specifically asked about in 1998. In 2003 this statistic was derived from the reported ownership shares. 
4. Use of computers by small businesses and type of use, by number of employees, industry, and age of firm, 1998 and 2003 surveys

Percent

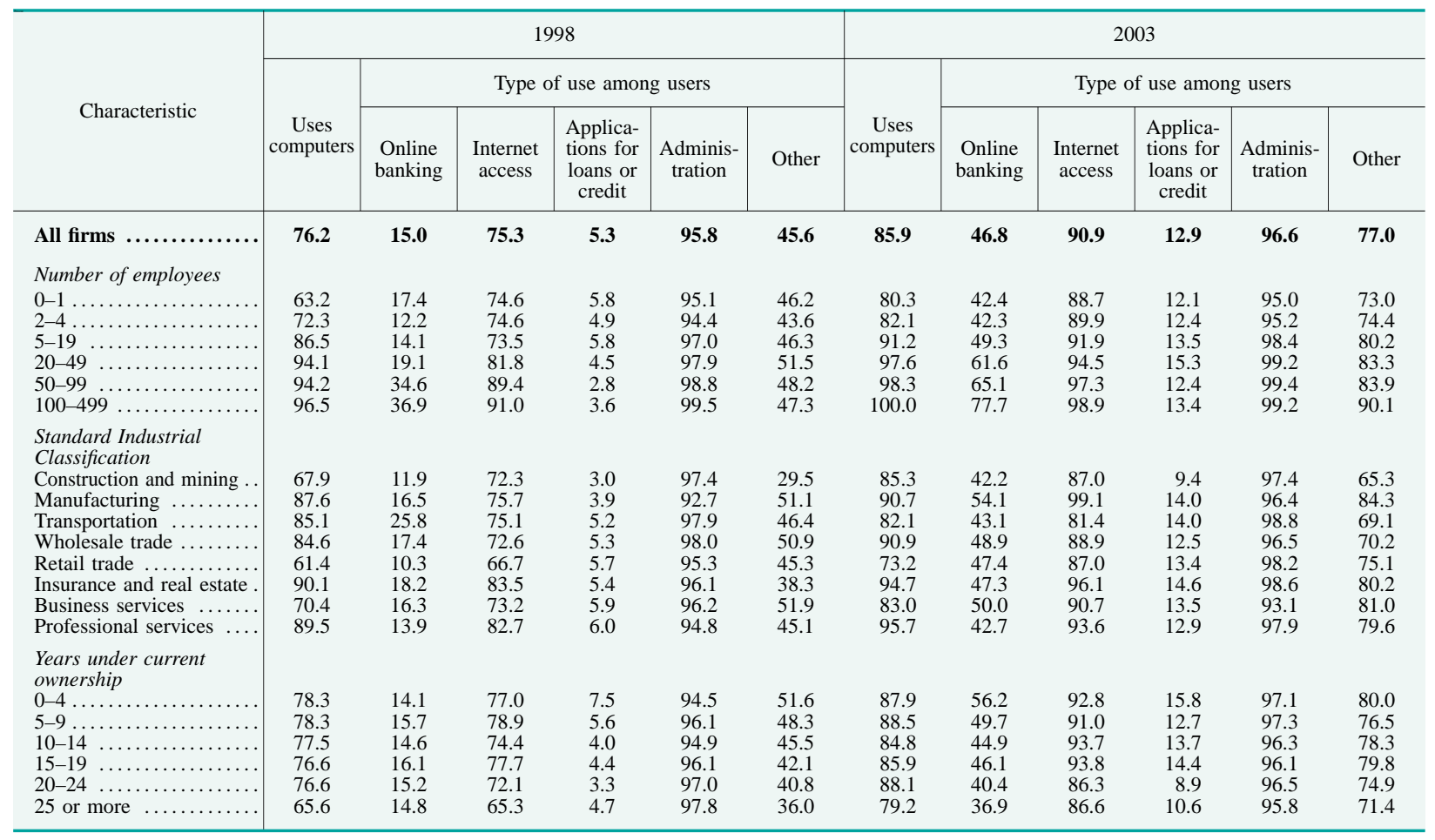

previous surveys, the female-owned firms tended to be younger and smaller in terms of employment, sales, and assets than those owned by males. They were also more likely to organize as proprietorships and less likely to organize as $\mathrm{S}$ corporations than male-owned firms. Female-owned firms were more likely to be engaged in professional and business services than male-owned firms and less likely to be engaged in construction, mining, and manufacturing.

Relative to white, non-Hispanic firms, nonwhite or Hispanic firms were younger and smaller in employment, sales, and assets and were more often organized as proprietorships. Similarly, nonwhite or Hispanic firms were also more likely to be engaged in business services and less likely to be engaged in construction and mining and insurance and real estate businesses.

\section{Computer Use within the Firm}

Use of a computer within a firm is one indicator of the extent of the firm's adoption of technological advances. In the 1998 survey, which was the first SSBF to ask firms about their use of computers, 76.2 percent reported using them (table 4). By 2003, the proportion had increased to 85.9 percent. Among the firms using computers, the proportion that used them for online banking rose between the two surveys from 15.0 percent to 46.8 percent; likewise, the proportion that used computers to apply for credit or loans also rose substantially, from 5.3 percent to 12.9 percent.

With the rise in prevalence of computer use came a rise in incidence and a narrowing in the variation of incidence across firm age and size. For example although the incidence of use still varied with the number of employees, the range in 2003 - 80 percent to 100 percent-was higher and narrower than that in 1998-63 percent to 97 percent. And although incidence of use varies inversely with firm age, the range of incidence by age also rose and narrowed between the two surveys.

\section{Nonstandard Work Arrangements}

The use of nonstandard work arrangements has been on the rise since at least the mid-1990s. For example, estimates from the February 1995 Current Population Survey indicate that 12.1 million workers (or 9.8 percent of the total) were independent contractors, on-call workers, temporary agency workers, or workers provided through contract firms. ${ }^{15}$ By 2005, estimates indicate that 14.8 million workers, or 10.7

15. Anne E. Polivka (1996), "Contingent and Alternative Work Arrangements, Defined," Monthly Labor Review, October, pp. 3-9. The Current Population Survey, conducted monthly by the U.S. Census Bureau for the U.S. Bureau of Labor Statistics, covers about 50,000 households (www.bls.census.gov/cps/cpsmain.htm). 
5. Use of nonstandard work arrangements by small businesses during a typical pay period, and types of arrangements, by number of employees and industry, 2003 survey

Percent except as noted

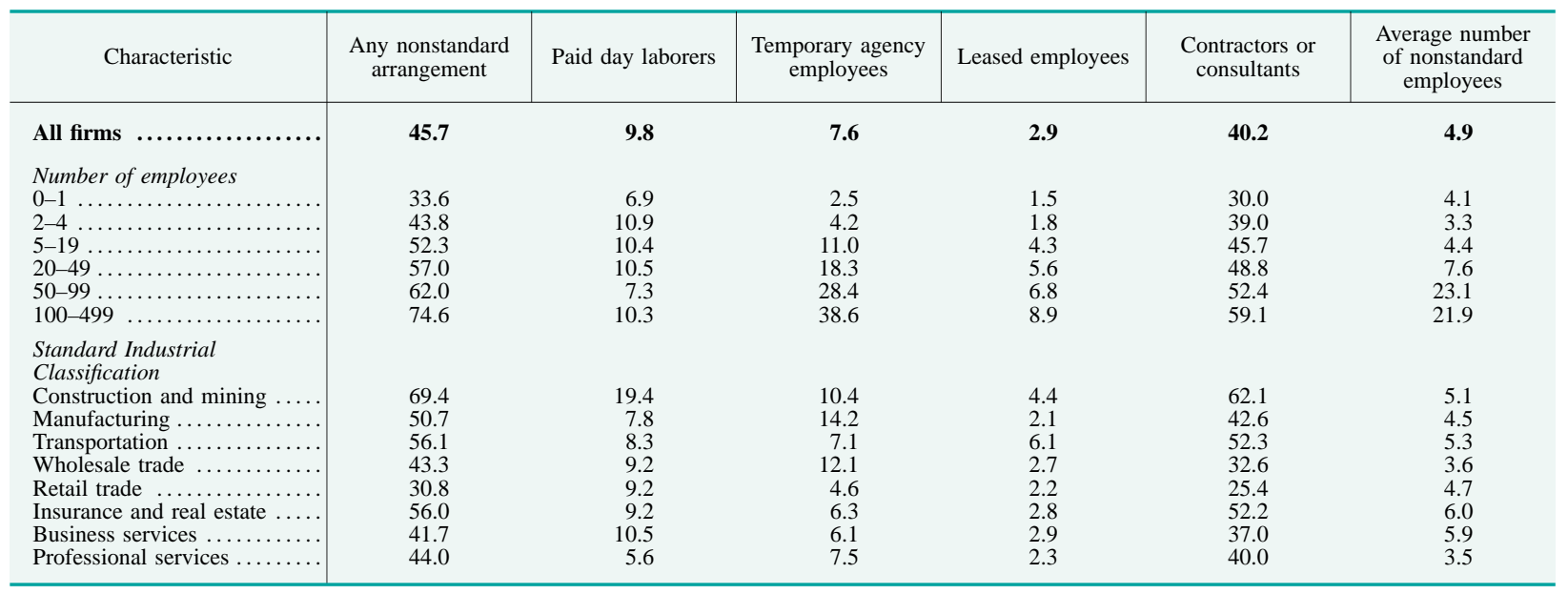

percent of total employment, fell into one of these groups. ${ }^{16}$

For the 2003 survey, the SSBF asked respondents for the first time about the use of nonstandard work arrangements. After a series of questions on the use of standard employees (both paid and unpaid), respondents were asked whether, during a typical pay period, they used any paid day laborers, temporary agency employees, workers from an employee-leasing firm, or contractors. About half of the firms reported using at least one of these arrangements.

The use of nonstandard work arrangements varied substantially by firm size. In general, the larger the firm, the more likely it was to have employed at least one worker in each of the nonstandard arrangements (table 5). Across all firm sizes, contractors and consultants were the most common types of nonstandard workers reported, a result consistent with statistics calculated from the employee side (refer to text notes 15 and 16). About 30 percent of the smallest firms used contractors and consultants, and about 59 percent of the very largest firms did so. Among firms using any nonstandard workers, the number generally increased with firm size.

The use of nonstandard work arrangements varied substantially by firm industry. At the extremes, nearly 70 percent of firms involved in construction and mining reported some type of nonstandard arrangement, whereas only 31 percent of retail trade firms reported doing so. Across all industries, contractors and consultants again were the most common types of nonstandard workers reported; by industry, the pro-

16. U.S. Bureau of Labor Statistics (2005), "Contingent and Alternative Employment Arrangements, February 2005," press release, July 27, www.bls.gov/news.release/conemp.nr0.htm. portion of firms that used contractors and consultants ranged from 25 percent in retail trade to 62 percent in construction and mining.

\section{TYPES OF FINANCIAL SERVICES USED BY SMALL BUSINESSES}

Firms were asked which of fourteen financial services they used at up to twenty institutions. ${ }^{17}$ The financial services can be grouped into three broad categories: (1) liquid asset accounts, which are checking and savings-type accounts, (2) credit lines, loans, and capital leases, which are lines of credit, mortgages used for business purposes, motor vehicle loans, equipment loans, capital leases, and miscellaneous or "other" loans, and (3) financial management services, which are transaction services, credit card and debit card processing services, cash management services, credit-related services, brokerage services, and trust and pension services. Loans from owners, credit cards, and trade credit are discussed separately and are not included in the tabulations for "any financial service" because no information was collected about the providers of these services.

Nearly all small businesses (about 96 percent) used at least one financial service in 2003, a finding

17. For this article, use of a financial service was measured by the percentage of small businesses using that service. Data on use that are based on dollar amounts or numbers of accounts will be available at a later date. However, previous analysis has shown that conclusions based on dollar amounts or on number of accounts are usually qualitatively very similar to conclusions based on percentages of firms. Further discussion is in Rebel A. Cole, John D. Wolken, and R. Louise Woodburn (1996), "Bank and Nonbank Competition for Small Business Credit: Evidence from the 1987 and 1993 National Surveys of Small Business Finances," Federal Reserve Bulletin, vol. 83 (November), pp. 983-95. 
6. Use of selected financial services by small businesses, by selected characteristics of firms, 2003 survey

A. Any service; liquid asset accounts; credit lines, loans, and capital leases

Percent

\begin{tabular}{|c|c|c|c|c|c|c|c|c|c|c|c|}
\hline \multirow{3}{*}{ Characteristic } & \multirow{3}{*}{$\begin{array}{c}\text { Any } \\
\text { service }^{1}\end{array}$} & \multirow{2}{*}{\multicolumn{3}{|c|}{ Liquid asset account }} & \multicolumn{7}{|c|}{ Credit line, loan, or capital lease } \\
\hline & & & & & \multirow{2}{*}{ Any } & \multirow{2}{*}{$\begin{array}{l}\text { Credit } \\
\text { line }\end{array}$} & \multicolumn{3}{|c|}{ Loan } & \multirow{2}{*}{$\begin{array}{l}\text { Capital } \\
\text { lease }\end{array}$} & \multirow{2}{*}{ Other } \\
\hline & & Any & Checking & Savings & & & Mortgage & Vehicle & Equipment & & \\
\hline \multicolumn{12}{|l|}{ All firms } \\
\hline $\begin{array}{l}2003 \\
1998 \ldots \ldots \ldots \ldots \ldots \ldots \ldots \ldots\end{array}$ & $\begin{array}{l}96.4 \\
96.1\end{array}$ & 95.0 & 94.6 & 22.1 & 60.4 & 34.3 & 13.3 & 25.5 & 10.3 & 8.7 & 10.1 \\
\hline $1998 \ldots \ldots \ldots \ldots \ldots \ldots \ldots$ & 96.1 & 94.4 & 94.0 & 22.2 & 55.0 & 27.7 & 13.2 & 20.5 & 9.9 & 10.6 & 9.8 \\
\hline \multicolumn{12}{|l|}{ Number of employees } \\
\hline & 96.9 & 95.1 & 94.8 & $\begin{array}{l}18.4 \\
18.6\end{array}$ & $\begin{array}{l}4.1 \\
53.9\end{array}$ & $\begin{array}{l}19.4 \\
27.2\end{array}$ & $\begin{aligned} 5.0 \\
12.6\end{aligned}$ & $\begin{array}{l}1.4 \\
220\end{array}$ & $\begin{array}{l}4.3 \\
5.2\end{array}$ & $\begin{array}{l}4.0 \\
6.6\end{array}$ & 7.1 \\
\hline $5-9 \ldots$ & 99.8 & 99.4 & 99.2 & 25.7 & 72.7 & 43.1 & 15.8 & 30.8 & 13.6 & 11.6 & 13.3 \\
\hline $10-19$ & 100.0 & 99.8 & 99.8 & 30.9 & 77.4 & 50.2 & 19.2 & 35.9 & 21.1 & 12.1 & 16.5 \\
\hline $20-49$ & 100.0 & 99.9 & 99.9 & 33.3 & 82.7 & 57.5 & 21.4 & 36.2 & 26.3 & 16.0 & 15.7 \\
\hline $50-99 \ldots$ & 100.0 & 98.7 & 98.5 & 41.1 & 87.4 & 68.0 & 18.6 & 36.5 & 27.6 & 22.9 & 16.5 \\
\hline $100-499$. & 100.0 & 100.0 & 100.0 & 36.5 & 93.8 & 82.3 & 28.0 & 35.9 & 32.6 & 27.9 & 18.6 \\
\hline \multicolumn{12}{|l|}{ Sales (thousands of dollars) } \\
\hline Less than $25 \ldots \ldots \ldots \ldots$ & 81.4 & 76.7 & 75.3 & 10.8 & 29.4 & 12.3 & 5.3 & 10.2 & $*$ & & 6.0 \\
\hline $25-49 \ldots \ldots$ & 97.2 & 94.1 & 93.7 & 19.6 & 45.6 & 14.1 & 8.9 & 17.3 & $*$ & 5.8 & 8.9 \\
\hline $50-99 \ldots$ & 97.6 & 96.7 & 96.2 & 16.7 & 49.5 & 24.2 & 10.1 & 23.0 & 7.4 & 3.6 & 5.3 \\
\hline $100-249$. & 98.9 & 98.3 & 98.3 & 19.1 & 59.9 & 29.2 & 13.3 & 21.9 & 8.0 & 9.1 & 9.1 \\
\hline $250-499$. & 99.6 & 99.2 & 98.9 & 22.9 & 70.7 & 39.8 & 19.1 & 27.9 & 10.0 & 10.4 & 12.5 \\
\hline $500-999 \ldots$. & 100.0 & 99.4 & 99.4 & 27.4 & 80.0 & 47.8 & 15.6 & 38.7 & 16.7 & 12.9 & 14.4 \\
\hline $1,000-2,499$ & 100.0 & 99.0 & 99.0 & 37.2 & 76.4 & 56.6 & 18.9 & 35.5 & 21.4 & 13.1 & 12.5 \\
\hline $2,500-4,999$ & 100.0 & 99.2 & 99.1 & 30.3 & 79.9 & 65.2 & 14.6 & 37.2 & 19.1 & 15.1 & 15.0 \\
\hline $5,000-9,999 \ldots$ & 100.0 & 100.0 & 100.0 & 34.7 & 90.4 & 65.6 & 22.3 & 49.4 & 28.4 & 16.3 & 14.1 \\
\hline 10,000 or more & 100.0 & 99.8 & 99.8 & 39.1 & 90.7 & 83.8 & 16.3 & 33.3 & 23.2 & 20.9 & 18.1 \\
\hline \multicolumn{12}{|l|}{ Assets (thousands of dollars) } \\
\hline Less than $25 \ldots \ldots \ldots \ldots \ldots$ & 90.0 & 86.8 & 86.2 & 13.3 & 39.0 & 16.2 & 4.3 & 16.2 & 3.3 & 5.2 & 6.1 \\
\hline $25-49 \ldots \ldots$ & 98.2 & 97.9 & 97.7 & 17.4 & 57.2 & 31.0 & 6.8 & 23.5 & 6.5 & 8.3 & 5.5 \\
\hline $50-99 \ldots$ & 99.8 & 98.5 & 98.0 & 22.9 & 66.2 & 32.8 & 13.8 & 26.9 & 9.1 & 9.4 & 13.4 \\
\hline $100-249$. & 99.1 & 98.4 & 98.4 & 25.9 & 67.2 & 37.1 & 16.3 & 31.4 & 12.5 & 7.9 & 10.3 \\
\hline 250-499. & 99.7 & 99.1 & 98.6 & 29.7 & 78.1 & 48.5 & 22.4 & 32.3 & 17.6 & 12.3 & 12.9 \\
\hline $500-999 \ldots$ & 100.0 & 99.7 & 99.7 & 27.5 & 79.0 & 55.7 & 23.8 & 30.5 & 16.5 & 12.0 & 19.7 \\
\hline $1,000-2,499$ & 100.0 & 100.0 & 100.0 & 38.7 & 82.1 & 62.2 & 27.3 & 39.5 & 24.6 & 12.5 & 12.2 \\
\hline $2,500-4,999 \ldots$ & 100.0 & 96.0 & 95.9 & 41.8 & 88.1 & 64.8 & 27.6 & 34.5 & 26.7 & 20.6 & 21.4 \\
\hline 5,000 or more & 100.0 & 100.0 & 100.0 & 32.8 & 80.3 & 66.6 & 28.8 & 28.0 & 17.9 & 15.9 & 14.0 \\
\hline \multicolumn{12}{|l|}{ Organizational form } \\
\hline Proprietorship ...... & 93.0 & 90.5 & 89.9 & 17.2 & 52.4 & 24.2 & 11.1 & 21.5 & 6.9 & 5.5 & 9.5 \\
\hline Partnership ... & 97.3 & 95.7 & 95.7 & 22.1 & 57.1 & 28.7 & 20.5 & 20.4 & 10.4 & 8.9 & 6.2 \\
\hline S corporation & 99.5 & 98.9 & 98.8 & 24.1 & 70.0 & 43.8 & 15.4 & 31.3 & 11.9 & 11.7 & 12.5 \\
\hline C corporation .... & 99.6 & 99.4 & 99.1 & 32.1 & 66.2 & 47.2 & 11.3 & 28.2 & 16.6 & 12.0 & 9.7 \\
\hline \multicolumn{12}{|l|}{$\begin{array}{l}\text { Standard Industrial } \\
\text { Classification }\end{array}$} \\
\hline Construction and mining & 96.5 & 95.0 & 95.0 & 23.9 & 70.8 & 44.6 & 13.6 & 43.9 & 16.5 & 6.3 & 7.5 \\
\hline Manufacturing ............. & 97.7 & 97.2 & 96.9 & 27.3 & 70.0 & 47.8 & 18.0 & 27.3 & 17.6 & 10.9 & 10.5 \\
\hline Transportation . . & 99.5 & 98.3 & 98.3 & 31.3 & 79.1 & 36.5 & 9.5 & 42.9 & 16.0 & 9.3 & 20.0 \\
\hline Wholesale trade & 98.8 & 96.7 & 96.3 & 22.7 & 62.6 & 49.5 & 13.1 & 30.1 & 7.1 & 7.8 & 9.6 \\
\hline Retail trade $\ldots \ldots \ldots \ldots \ldots$ & 97.4 & 96.3 & 96.3 & 17.6 & 58.9 & 32.8 & 14.7 & 19.7 & 9.2 & 7.3 & 14.0 \\
\hline Insurance and real estate & 96.3 & 95.5 & 95.1 & 28.6 & 59.2 & 28.8 & 23.1 & 21.1 & $*$ & 5.3 & 5.4 \\
\hline Business services ....... & 94.9 & 93.2 & 92.4 & 19.9 & 56.4 & 28.4 & 11.2 & 25.0 & 7.9 & 7.4 & 10.1 \\
\hline Professional services $\ldots \ldots \ldots$ & 95.9 & 93.9 & 93.5 & 22.0 & 54.1 & 29.4 & 10.1 & 17.3 & 10.2 & 13.5 & 8.1 \\
\hline \multicolumn{12}{|l|}{ Years under current ownership } \\
\hline & $\begin{array}{l}96.1 \\
95.8\end{array}$ & $\begin{array}{l}94.3 \\
94.4\end{array}$ & $\begin{array}{l}93.8 \\
93.9\end{array}$ & $\begin{array}{l}16.8 \\
20.0\end{array}$ & $\begin{array}{l}61.8 \\
60.0\end{array}$ & $\begin{array}{l}30.2 \\
30.2\end{array}$ & $\begin{array}{l}13.3 \\
11.7\end{array}$ & $\begin{array}{l}21.2 \\
24.9\end{array}$ & $\begin{array}{r}7.0 \\
11.1\end{array}$ & $\begin{array}{r}1.0 \\
7.1\end{array}$ & $\begin{array}{l}15.4 \\
12.8\end{array}$ \\
\hline $\begin{array}{l}5-9 \\
10-14 \ldots \ldots \ldots \ldots \ldots \ldots \ldots \ldots \ldots \ldots \\
\end{array}$ & $\begin{array}{l}93.8 \\
98.1\end{array}$ & $\begin{array}{l}96.4 \\
96.5\end{array}$ & 96.1 & 25.7 & 60.4 & 35.1 & 14.6 & 28.0 & 10.8 & 10.4 & 7.7 \\
\hline $15-19 \ldots \ldots$ & 94.7 & 92.5 & 92.4 & 20.3 & 61.4 & 40.0 & 12.5 & 28.8 & 11.4 & 9.4 & 7.5 \\
\hline $20-24 \ldots \ldots$ & 96.0 & 94.6 & 94.6 & 22.8 & 58.1 & 33.9 & 13.7 & 25.2 & 12.6 & 8.4 & 5.4 \\
\hline 25 or more & 97.8 & 97.0 & 96.8 & 28.6 & 60.2 & 39.3 & 14.5 & 26.9 & 10.4 & 6.3 & 7.7 \\
\hline
\end{tabular}

essentially the same as in 1998 (table 6.A). In general, use increased with firm size, and nearly all firms with at least five employees, or with sales of at least $\$ 250,000$, or with assets of at least $\$ 50,000$ used some financial service. About 11 percent of firms with one worker used no financial service in 2003. ${ }^{18}$

18. An alternative to incidence as a measure of intensity of use is the number of distinct services (which range from 0 to 14 for each source)
Proprietorships were less likely than corporations or partnerships to use any financial service. The difference may be due to the tendency of many proprietorships to commingle business and personal finances; for example, the owners may use personal

across all financial suppliers for each firm. The average number of services used by small businesses in 2003 was 3.9. One-half of the firms used 3 or fewer services, one-third used 2 or fewer, one-fourth used 5 or more, and 2.5 percent used 10 or more. 
6.-Continued

A. Any service; liquid asset accounts; credit lines, loans, and capital leases Percent

\begin{tabular}{|c|c|c|c|c|c|c|c|c|c|c|c|}
\hline \multirow{3}{*}{ Characteristic } & \multirow{3}{*}{$\begin{array}{c}\text { Any } \\
\text { service }^{1}\end{array}$} & \multirow{2}{*}{\multicolumn{3}{|c|}{ Liquid asset account }} & \multicolumn{7}{|c|}{ Credit line, loan, or capital lease } \\
\hline & & & & & \multirow{2}{*}{ Any } & \multirow{2}{*}{$\begin{array}{l}\text { Credit } \\
\text { line }\end{array}$} & \multicolumn{3}{|c|}{ Loan } & \multirow{2}{*}{$\begin{array}{c}\text { Capital } \\
\text { lease }\end{array}$} & \multirow{2}{*}{ Other } \\
\hline & & Any & Checking & Savings & & & Mortgage & Vehicle & Equipment & & \\
\hline \multicolumn{12}{|l|}{ Census area of main office } \\
\hline Northeast ............... & 95.5 & 94.9 & 94.9 & 19.2 & 57.8 & 33.8 & 11.5 & 21.4 & 9.1 & 7.6 & 9.6 \\
\hline New England & 97.9 & 97.5 & 97.5 & 18.3 & 62.3 & 35.5 & 13.6 & 28.2 & 10.8 & 6.9 & 10.7 \\
\hline Middle Atlantic . & 94.4 & 93.8 & 93.8 & 19.7 & 55.9 & 33.0 & 10.6 & 18.5 & 8.3 & 7.9 & 9.2 \\
\hline Midwest . . . . . . . . . & 98.4 & 96.5 & 96.1 & 26.8 & 67.0 & 41.1 & 17.1 & 26.5 & 12.3 & 9.6 & 9.7 \\
\hline East North Central & 98.0 & 95.8 & 95.3 & 26.3 & 67.1 & 39.4 & 19.2 & 25.7 & 10.6 & 10.1 & 11.0 \\
\hline West North Central .. & 99.3 & 98.1 & 97.8 & 27.8 & 66.7 & 44.4 & 12.9 & 28.2 & 15.9 & 8.5 & 7.0 \\
\hline South ......... & 96.5 & 94.7 & 94.1 & 17.7 & 61.8 & 31.9 & 14.1 & 28.9 & 10.4 & 7.8 & 10.5 \\
\hline South Atlantic ...... & 97.5 & 95.9 & 94.8 & 17.6 & 63.9 & 31.3 & 13.9 & 32.0 & 10.5 & 8.5 & 9.1 \\
\hline East South Central . & 94.9 & 93.1 & 93.1 & 20.9 & 57.0 & 31.0 & 9.2 & 21.3 & 9.5 & 6.4 & 14.9 \\
\hline West South Central & 95.5 & 93.3 & 93.3 & 16.2 & 60.5 & 33.3 & 16.8 & 27.1 & 10.7 & 7.4 & 10.9 \\
\hline West ....... & 95.4 & 94.1 & 93.8 & 26.8 & 54.8 & 32.2 & 10.2 & 23.1 & 9.4 & 10.0 & 10.3 \\
\hline Mountain & 95.6 & 94.2 & 93.8 & 23.4 & 62.4 & 36.4 & 14.5 & 28.9 & 11.9 & 9.2 & 14.1 \\
\hline Pacific ............ & 95.4 & 94.0 & 93.8 & 28.4 & 51.4 & 30.3 & 8.3 & 20.5 & 8.2 & 10.4 & 8.6 \\
\hline \multicolumn{12}{|l|}{ Urbanization at main office } \\
\hline Urban..........$\ldots \ldots \ldots$ & 96.5 & 95.0 & 94.6 & 22.4 & 60.2 & 33.8 & 12.2 & 24.9 & 9.4 & 9.4 & 10.1 \\
\hline Rural ......................... & 96.2 & 95.1 & 94.9 & 21.3 & 61.5 & 36.2 & 17.6 & 27.9 & 13.7 & 6.0 & 10.4 \\
\hline \multicolumn{12}{|l|}{ Number of offices } \\
\hline One ............. & 95.9 & 94.4 & 94.0 & 21.0 & 58.1 & 31.8 & 12.9 & 24.9 & 9.7 & 7.7 & 9.0 \\
\hline Two ............ & 99.2 & 98.4 & 98.1 & 27.5 & 70.4 & 45.5 & 13.2 & 27.4 & 11.5 & 14.1 & 18.5 \\
\hline Three or more ........ & 100.0 & 99.2 & 99.2 & 32.7 & 82.7 & 56.8 & 21.0 & 33.9 & 18.4 & 17.2 & 14.8 \\
\hline \multicolumn{12}{|l|}{ Sales area } \\
\hline $\begin{array}{l}\text { Primarily within the } \\
\text { United States } \ldots \ldots \text {. }\end{array}$ & 96.4 & 95.0 & 94.6 & 22.1 & 60.8 & 34.4 & 13.5 & 25.7 & 10.4 & 8.7 & 10.1 \\
\hline International or global $\ldots .$. & 97.1 & 94.3 & 94.3 & 23.8 & $\begin{array}{l}00.8 \\
51.8\end{array}$ & $\begin{array}{l}34.4 \\
32.2\end{array}$ & $\begin{array}{r}13.3 \\
8.4\end{array}$ & 22.6 & $\begin{array}{r}10.4 \\
8.6\end{array}$ & $\begin{array}{l}8.1 \\
8.7\end{array}$ & $\begin{array}{l}10.1 \\
11.5\end{array}$ \\
\hline \multicolumn{12}{|l|}{ Management } \\
\hline By owner ... & 96.2 & 94.7 & 94.3 & 21.5 & 59.9 & 34.1 & 12.7 & 25.5 & 10.1 & 8.4 & 10.0 \\
\hline Hired .... & 99.9 & 99.9 & 99.9 & 31.8 & 68.3 & 35.1 & 21.5 & 26.5 & 13.7 & 12.6 & 11.6 \\
\hline \multicolumn{12}{|l|}{$\begin{array}{l}\text { Race, ethnicity, and sex of } \\
\text { maiority owners }\end{array}$} \\
\hline Nonwhite or Hispanic ..... & 95.5 & 92.8 & 92.0 & 17.8 & 54.8 & 25.6 & 11.6 & 25.7 & 5.5 & 5.9 & 12.5 \\
\hline Non-Hispanic white ....... & 96.5 & 95.3 & 95.0 & 22.8 & 60.9 & 35.5 & 13.3 & 25.4 & 11.0 & 9.0 & 9.6 \\
\hline White . & 96.5 & 95.1 & 94.8 & 22.5 & 61.0 & 35.4 & 13.1 & 25.7 & 10.7 & 8.9 & 9.6 \\
\hline Black $\ldots \ldots \ldots \ldots \ldots$ & 91.7 & 89.8 & 88.0 & 19.5 & 47.5 & 12.1 & 10.6 & 25.4 & $*$ & 8.9 & 11.9 \\
\hline \multicolumn{12}{|l|}{ Asian, Native Hawaiian, or } \\
\hline \multicolumn{12}{|l|}{$\begin{array}{l}\text { other Pacific lslander } \\
\text { American Indian or Alaska }\end{array}$} \\
\hline Native...$\ldots \ldots \ldots \ldots$. & 92.7 & 90.9 & 88.5 & $*$ & 63.1 & 35.5 & * & 28.2 & $*$ & $*$ & * \\
\hline Hispanic $\ldots \ldots \ldots \ldots \ldots$ & 94.9 & 90.4 & 90.4 & 17.5 & 61.1 & 31.6 & 13.6 & 33.3 & 5.8 & 6.7 & 11.9 \\
\hline Non-Hispanic ......... & 96.5 & 95.2 & 94.8 & 22.3 & 60.3 & 34.3 & 13.2 & 25.2 & 10.5 & 8.8 & 10.0 \\
\hline \multirow{3}{*}{ 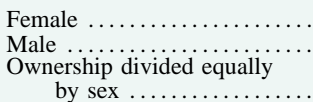 } & 92.3 & 89.6 & 89.0 & 19.9 & 47.6 & 23.4 & 10.7 & 19.6 & 8.4 & 7.2 & 7.3 \\
\hline & 97.4 & 96.3 & 96.0 & 22.0 & 64.4 & 38.1 & 13.0 & 27.7 & 11.0 & 9.1 & 11.1 \\
\hline & 98.6 & 97.8 & 97.6 & 26.3 & 62.3 & 32.9 & 18.8 & 25.1 & 10.1 & 9.0 & 10.1 \\
\hline
\end{tabular}

NotE: For definitions of services, refer to text; for definition of sales areas, refer to table 2, note 3 .

1. Memo items in table 6.B are excluded from these data.

*Fewer than fifteen observations.

savings and checking accounts for business purposes. ${ }^{19}$ Also, firms whose ownership was black, American Indian or Alaska Native, Hispanic, or female were less likely to use any financial service than were firms whose ownership was white, nonHispanic, or male.

19. Respondents were asked to count as a business service any personal account that was used at least 50 percent of the time for business purposes. Most of the firms that reported using no financial services were extremely small; it is possible that the owners of those firms used personal accounts for business purposes but did so less than 50 percent of the time.

\section{Liquid Asset Services}

Most small businesses (95 percent) had a checking account in 2003, the same percentage as used any liquid asset account, that is, a checking or savings account (table 6.A). ${ }^{20}$ Because a checking account

20. Checking accounts were defined as accounts with unlimited check-writing privileges and included those in credit unions (share draft accounts). Money market accounts, including money market deposit accounts, were considered to be checking accounts only if they offered unlimited check-writing privileges. Savings accounts were defined as passbook savings, credit union share accounts, certificates 
6. Use of selected financial services by small businesses, by selected characteristics of firms, 2003 survey

B. Financial management services

Percent

\begin{tabular}{|c|c|c|c|c|c|c|c|c|c|c|c|c|}
\hline \multirow{4}{*}{ Characteristic } & \multicolumn{7}{|c|}{ Financial management service } & \multicolumn{5}{|c|}{ Мемо } \\
\hline & \multirow{3}{*}{ Any } & \multirow{3}{*}{$\begin{array}{l}\text { Trans- } \\
\text { action }\end{array}$} & \multirow{3}{*}{$\begin{array}{c}\text { Credit } \\
\text { and } \\
\text { debit } \\
\text { card } \\
\text { processing }\end{array}$} & \multirow{3}{*}{$\begin{array}{c}\text { Cash } \\
\text { manage- } \\
\text { ment }\end{array}$} & \multirow{3}{*}{$\begin{array}{l}\text { Credit- } \\
\text { related }\end{array}$} & \multirow{3}{*}{ Brokerage } & \multirow{3}{*}{$\begin{array}{l}\text { Trust } \\
\text { and } \\
\text { pension }\end{array}$} & \multicolumn{4}{|c|}{ Nontraditional credit } & \multirow{3}{*}{$\begin{array}{c}\text { Traditional } \\
\text { and non- } \\
\text { traditional } \\
\text { credit }\end{array}$} \\
\hline & & & & & & & & \multirow{2}{*}{$\begin{array}{l}\text { Loan } \\
\text { from } \\
\text { owner }\end{array}$} & \multicolumn{2}{|c|}{ Credit card } & \multirow{2}{*}{$\begin{array}{l}\text { Trade } \\
\text { credit }\end{array}$} & \\
\hline & & & & & & & & & Personal & Business & & \\
\hline All firms & & & & & & & & & & & & \\
\hline $2003 \ldots \ldots$ & 64.7 & 38.9 & 37.2 & 6.7 & 5.0 & 5.6 & 17.2 & 30.3 & 46.7 & 48.1 & 60.1 & 92.9 \\
\hline $1998 \ldots \ldots \ldots \ldots \ldots$ & 49.8 & 41.1 & n.a. & 5.2 & 3.1 & 4.3 & 12.6 & 28.1 & 46.0 & 34.1 & 61.9 & 89.5 \\
\hline Number of employees & & & & & & & & & & & & \\
\hline $0-1 \ldots \ldots \ldots \ldots \ldots \ldots$ & 41.5 & 25.2 & & 2.7 & $*$ & 2.6 & 8.0 & 25.7 & 48.6 & 32.0 & 35.7 & 83.8 \\
\hline & $\begin{array}{l}59.6 \\
77.4\end{array}$ & 35.0 & $\begin{array}{l}33.7 \\
47.9\end{array}$ & $\begin{array}{l}3.8 \\
8.3\end{array}$ & $\begin{array}{l}4.1 \\
6.9\end{array}$ & $\begin{array}{l}5.0 \\
7.9\end{array}$ & 10.3 & 27.0 & $\begin{array}{l}48.1 \\
478\end{array}$ & 45.7 & 55.9 & $\begin{array}{l}93.3 \\
96.6\end{array}$ \\
\hline $\begin{array}{l}5-9 \ldots \ldots \ldots \ldots \ldots \\
10-19\end{array}$ & $\begin{array}{l}77.4 \\
84.4\end{array}$ & 45.1 & $\begin{array}{l}47.9 \\
57.3\end{array}$ & $\begin{array}{l}8.3 \\
8.1\end{array}$ & $\begin{array}{l}6.9 \\
4.9\end{array}$ & $\begin{array}{l}7.9 \\
7.0\end{array}$ & 20.5 & 33.3 & 47.8 & 56.8 & 71.6 & 96.6 \\
\hline $\begin{array}{l}10-19 \ldots \ldots \ldots \ldots \\
20-49\end{array}$ & $\begin{array}{l}84.4 \\
901\end{array}$ & $\begin{array}{l}49.9 \\
610\end{array}$ & $\begin{array}{l}51.3 \\
563\end{array}$ & & $\begin{array}{l}4.9 \\
9.0\end{array}$ & 7.0 & 29.2 & 31.3 & 45.6 & 59.7 & 80.4 & 97.3 \\
\hline $\begin{array}{l}20-49 \ldots \\
50-99 \ldots\end{array}$ & $\begin{array}{l}9.1 \\
88.3\end{array}$ & $\begin{array}{l}6.0 \\
61.3\end{array}$ & 51.5 & $\begin{array}{l}15.0 \\
37.8\end{array}$ & 15.8 & $\begin{array}{r}7.1 \\
12.8\end{array}$ & $\begin{array}{l}41.9 \\
595\end{array}$ & 36.0 & $\begin{array}{l}34.4 \\
346\end{array}$ & 61.8 & $\begin{array}{l}85.0 \\
88.5\end{array}$ & 99.8 \\
\hline & $\begin{array}{l}0.3 \\
96.1\end{array}$ & 70.3 & 50.2 & 50.1 & $\begin{array}{l}26.0 \\
26.7\end{array}$ & $\begin{array}{l}12.8 \\
13.3\end{array}$ & $\begin{array}{l}59.9 \\
68.4\end{array}$ & $\begin{array}{l}32.9 \\
28.4\end{array}$ & $\begin{array}{l}34.6 \\
32.2\end{array}$ & $\begin{array}{l}63.5 \\
71.5\end{array}$ & $\begin{array}{l}88.5 \\
85.4\end{array}$ & $\begin{array}{l}98.3 \\
98.9\end{array}$ \\
\hline $\begin{array}{l}\text { Sales (thousands } \\
\text { of dollars) }\end{array}$ & & & & & & & & & & & & \\
\hline Less than $25 \ldots$ & 36.3 & 23.2 & 15.4 & * & * & * & $*$ & 22.3 & 48.1 & 25.7 & 27.7 & 79.2 \\
\hline $25-49 \ldots \ldots$ & 49.7 & 30.5 & 24.5 & * & * & $*$ & 7.5 & 33.5 & 51.7 & 34.1 & 40.2 & 89.2 \\
\hline $50-99 \ldots$ & 49.6 & 29.1 & 25.6 & $*$ & $*$ & $*$ & 10.5 & 34.9 & 46.5 & 41.0 & 46.3 & 91.4 \\
\hline $100-249$ & 62.6 & 34.3 & 37.5 & 3.7 & 4.6 & 4.4 & 10.1 & 28.1 & 49.9 & 48.2 & 61.0 & 94.5 \\
\hline $250-499$ & 74.7 & 43.9 & 48.3 & 7.4 & 4.2 & 6.7 & 15.6 & 27.7 & 49.4 & 54.9 & 70.0 & 96.3 \\
\hline $500-999 \ldots$ & 81.2 & 49.4 & 53.9 & 8.4 & 7.6 & 7.1 & 20.7 & 27.7 & 44.1 & 62.5 & 82.2 & 98.4 \\
\hline $1,000-2,499$ & 87.2 & 51.8 & 48.8 & 9.2 & 5.9 & 9.4 & 40.5 & 36.3 & 40.9 & 63.6 & 79.3 & 98.7 \\
\hline $2,500-4,999$ & 92.2 & 58.4 & $\begin{array}{l}4.0 \\
56.7\end{array}$ & 17.2 & 15.1 & 12.6 & 47.6 & $\begin{array}{l}38.5 \\
38.7\end{array}$ & 39.7 & $\begin{array}{l}61.9 \\
61.9\end{array}$ & 87.6 & $\begin{array}{r}70.1 \\
100.0\end{array}$ \\
\hline $5,000-9,999 \ldots$ & 91.7 & 65.2 & 39.2 & 19.7 & 14.1 & 15.7 & 52.6 & 26.9 & 30.8 & 63.3 & 83.6 & 99.5 \\
\hline 10,000 or more & 94.9 & 75.2 & 50.4 & 50.5 & 27.5 & 17.9 & 68.4 & 30.0 & 35.8 & 68.9 & 89.8 & 99.9 \\
\hline $\begin{array}{l}\text { Assets (thousands } \\
\text { of dollars) }\end{array}$ & & & & & & & & & & & & \\
\hline Less than $25 \ldots$. & 43.2 & 25.1 & 20.8 & 2.7 & * & 2.4 & 6.5 & 23.8 & 47.3 & 35.9 & 38.4 & 85.5 \\
\hline $25-49$. & 60.3 & 37.0 & 32.3 & $*$ & $*$ & $*$ & 11.6 & 28.1 & 47.4 & 48.1 & 57.6 & 93.5 \\
\hline $50-99 \ldots$ & 70.5 & 42.6 & 43.5 & 4.2 & 3.5 & 5.3 & 14.8 & 28.1 & 51.4 & 47.5 & 64.8 & 95.4 \\
\hline $100-249$ & 75.5 & 42.7 & 48.9 & 5.7 & 5.5 & 7.4 & 18.7 & 31.5 & 43.8 & 53.4 & 68.1 & 96.0 \\
\hline $250-499$ & 77.4 & 43.7 & 48.6 & 8.5 & 6.4 & 9.2 & 23.3 & 34.1 & 43.8 & 61.4 & 76.2 & 98.9 \\
\hline $500-999 \ldots$ & 84.9 & 52.1 & 52.2 & 7.5 & 11.8 & 10.1 & 30.0 & 37.8 & 47.7 & 53.6 & 76.2 & 96.7 \\
\hline $1,000-2,499$ & 83.0 & 53.2 & 48.8 & 16.4 & 13.5 & 9.7 & 37.4 & 31.8 & 50.5 & 64.7 & 84.5 & 99.0 \\
\hline $2,500-4,999 \ldots$ & $\begin{array}{l}0.0 \\
88.2\end{array}$ & $\begin{array}{l}62.7 \\
62.7\end{array}$ & $\begin{array}{l}4.0 \\
49.3\end{array}$ & $\begin{array}{l}10.4 \\
22.2\end{array}$ & 19.6 & 12.2 & $\begin{array}{l}3.4 \\
48.1\end{array}$ & 30.3 & 35.5 & $\begin{array}{l}0.1 \\
61.9\end{array}$ & 86.0 & 99.1 \\
\hline 5,000 or more . & 95.5 & 78.6 & 36.3 & 51.5 & 25.3 & 16.1 & 56.7 & 34.6 & 33.6 & 55.8 & 85.2 & 98.5 \\
\hline Organizational form & & & & & & & & & & & & \\
\hline Proprietorship ....... & 52.3 & 30.4 & 28.3 & 3.1 & 2.1 & 3.5 & 10.1 & & 52.3 & 35.1 & 46.9 & 88.3 \\
\hline Partnership ... & 70.4 & 44.4 & 34.7 & 7.6 & 6.6 & 9.5 & 13.1 & 25.2 & 41.7 & 46.9 & 58.5 & 93.9 \\
\hline $\mathrm{S}$ corporation . & 74.9 & 45.6 & 47.1 & 8.5 & 6.8 & 5.5 & 22.4 & 31.3 & 43.6 & 61.6 & 71.2 & 97.4 \\
\hline C corporation & 76.8 & 46.9 & 44.5 & 12.6 & 8.6 & 9.7 & 29.0 & 31.1 & 39.6 & 58.9 & 76.1 & 96.8 \\
\hline $\begin{array}{l}\text { Standard Industrial } \\
\text { Classification }\end{array}$ & & & & & & & & & & & & \\
\hline Construction and mining & 45.5 & 28.4 & 13.9 & 4.2 & 6.1 & 3.1 & 15.2 & 28.3 & 44.7 & 52.1 & 80.5 & 95.0 \\
\hline Manufacturing .......... & 67.2 & 45.1 & 36.5 & 10.0 & 11.9 & 5.1 & 19.7 & 39.8 & 47.1 & 54.8 & 76.0 & 97.0 \\
\hline Transportation .. & 56.7 & 33.5 & 37.5 & 8.4 & 13.2 & $*$ & 14.0 & 28.3 & 41.4 & 51.8 & 60.7 & 96.9 \\
\hline Wholesale trade & 73.6 & 44.6 & 48.2 & 7.5 & 8.2 & 5.9 & 22.5 & 34.1 & $\begin{array}{l}1.4 \\
46.7\end{array}$ & 54.4 & 72.2 & 94.1 \\
\hline Retail trade ..... & 82.4 & 46.8 & 72.1 & 4.9 & 3.4 & 2.4 & 11.5 & 38.2 & 47.9 & 45.0 & 67.4 & 91.4 \\
\hline Insurance and real estate & 63.4 & 40.9 & 9.3 & 15.3 & 6.9 & 13.6 & 19.9 & 17.7 & 47.4 & 43.0 & 45.9 & 89.8 \\
\hline Business services ....... & 62.3 & 35.7 & 38.5 & 6.1 & 3.4 & 3.9 & 11.6 & 27.4 & 45.2 & 47.0 & 52.5 & 91.5 \\
\hline Professional services .. & 61.7 & 38.6 & 24.8 & 5.6 & 2.1 & 9.9 & 27.4 & 26.2 & 48.9 & 47.1 & 46.8 & 93.5 \\
\hline $\begin{array}{l}\text { Years under current } \\
\text { ownership }\end{array}$ & & & & & & & & & & & & \\
\hline & 59.4 & 38.3 & 37.9 & 4.5 & 4.3 & 3.1 & 8.5 & 34.4 & 45.2 & 46.6 & 47.8 & 91.1 \\
\hline $5-9$. & 68.9 & 42.8 & 40.7 & 5.8 & 5.5 & 2.9 & 14.3 & 28.9 & 44.6 & 49.7 & 61.0 & 93.1 \\
\hline $10-14$ & 67.8 & 39.7 & 39.4 & 7.0 & 5.3 & 7.5 & 20.4 & 30.5 & 49.8 & 48.7 & 63.0 & 94.3 \\
\hline $15-19$. & 67.0 & 38.7 & 38.0 & 9.0 & 4.0 & 6.8 & 21.1 & 32.1 & 48.5 & 52.3 & 62.3 & 92.6 \\
\hline $20-24 \ldots \ldots \ldots$ & 63.0 & 37.3 & 33.9 & 6.8 & 3.2 & 7.8 & 23.7 & 28.1 & 46.9 & 50.7 & 62.8 & 92.1 \\
\hline 25 or more ...... & 62.5 & 35.4 & 31.7 & 8.1 & 6.6 & 8.1 & 20.9 & 27.2 & 46.6 & 43.1 & 67.0 & 94.5 \\
\hline
\end{tabular}

of deposit, and other time deposits; also considered to be savings accounts were money market accounts that were limited in either the number or the amount of checks that could be written. In comparison with small businesses, 91.4 percent of households in 2004 had some type of transaction account (checking account, savings account, money market deposit account, money market mutual fund, or call account at a brokerage). More information is available in Brian $\mathrm{K}$. (including a share draft account at a credit union) is a vehicle for paying suppliers and depositing sales receipts, it is not surprising that the reported use of

Bucks, Arthur B. Kennickell, and Kevin B. Moore (2006), "Recent Changes in U.S. Family Finances: Evidence from the 2001 and 2004 Survey of Consumer Finances," Federal Reserve Bulletin, vol. 92, pp. A1-A38. 


\section{6.-Continued}

B. Financial management services

Percent

\begin{tabular}{|c|c|c|c|c|c|c|c|c|c|c|c|c|}
\hline \multirow{4}{*}{ Characteristic } & \multicolumn{7}{|c|}{ Financial management service } & \multicolumn{5}{|c|}{ Мемо } \\
\hline & \multirow{3}{*}{ Any } & \multirow{3}{*}{$\begin{array}{l}\text { Trans- } \\
\text { action }\end{array}$} & \multirow{3}{*}{$\begin{array}{c}\text { Credit } \\
\text { and } \\
\text { debit } \\
\text { card } \\
\text { processing }\end{array}$} & \multirow{3}{*}{$\begin{array}{c}\text { Cash } \\
\text { manage- } \\
\text { ment }\end{array}$} & \multirow{3}{*}{$\begin{array}{l}\text { Credit- } \\
\text { related }\end{array}$} & \multirow{3}{*}{ Brokerage } & \multirow{3}{*}{$\begin{array}{l}\text { Trust } \\
\text { and } \\
\text { pension }\end{array}$} & \multicolumn{4}{|c|}{ Nontraditional credit } & \multirow{3}{*}{$\begin{array}{c}\text { Traditional } \\
\text { and non- } \\
\text { traditional } \\
\text { credit }\end{array}$} \\
\hline & & & & & & & & \multirow{2}{*}{$\begin{array}{l}\text { Loan } \\
\text { from } \\
\text { owner }\end{array}$} & \multicolumn{2}{|c|}{ Credit card } & \multirow{2}{*}{$\begin{array}{l}\text { Trade } \\
\text { credit }\end{array}$} & \\
\hline & & & & & & & & & Personal & Business & & \\
\hline \multicolumn{13}{|l|}{$\begin{array}{l}\text { Census area } \\
\text { of main office }\end{array}$} \\
\hline Northeast ...... & 65.2 & 39.6 & 33.6 & 4.9 & 3.4 & 4.5 & 18.9 & 34.9 & 45.1 & 44.7 & 59.3 & 92.6 \\
\hline New England .. & 67.2 & 42.3 & 35.5 & 3.7 & 3.1 & $*$ & 21.5 & 41.7 & 53.1 & 48.0 & 69.7 & 98.5 \\
\hline Middle Atlantic & 64.3 & 38.4 & 32.7 & 5.4 & 3.5 & 5.1 & 17.7 & 32.2 & 41.7 & 43.2 & 54.9 & 90.1 \\
\hline Midwest $\ldots \ldots \ldots \ldots$ & 67.2 & 38.2 & 38.4 & 10.0 & 6.3 & 6.8 & 22.1 & 28.1 & 46.3 & 50.9 & 65.8 & 94.6 \\
\hline East North Central & 64.7 & 37.6 & 36.7 & 9.7 & 6.4 & 8.6 & 21.8 & 30.0 & 40.8 & 53.2 & 63.8 & 94.6 \\
\hline West North Central . & 72.4 & 39.5 & 41.9 & 10.6 & 6.1 & 3.0 & 22.9 & 24.5 & 57.5 & 46.1 & 70.0 & 94.6 \\
\hline South ........... & 65.0 & 40.0 & 38.8 & 6.5 & 5.7 & 5.0 & 14.3 & 29.4 & 45.0 & 47.0 & 59.6 & 92.5 \\
\hline South Atlantic ..... & 68.9 & 41.5 & 43.3 & 6.7 & 5.6 & 4.5 & 15.9 & 29.5 & 44.9 & 53.9 & 61.3 & 94.3 \\
\hline East South Central . & 58.0 & 34.4 & 33.8 & 7.0 & 5.4 & 5.2 & 13.7 & 26.6 & 37.1 & 41.4 & 57.4 & 88.1 \\
\hline West South Central & 61.7 & 40.0 & 33.2 & 5.8 & 6.0 & 5.7 & 11.6 & 30.3 & 49.0 & 37.3 & 57.5 & 91.4 \\
\hline West $\ldots \ldots \ldots \ldots \ldots$ & 61.9 & 37.5 & 37.0 & 5.4 & 4.1 & 6.4 & 15.6 & 30.1 & 50.7 & 50.2 & 56.4 & 92.4 \\
\hline Mountain & 64.2 & 43.3 & 38.3 & 7.2 & 5.5 & 5.1 & 11.8 & 25.4 & 50.8 & 53.4 & 61.2 & 94.3 \\
\hline Pacific ... & 60.8 & 34.9 & 36.4 & 4.6 & 3.4 & 7.0 & 17.3 & 32.8 & 50.7 & 48.7 & 54.1 & 91.5 \\
\hline \multicolumn{13}{|l|}{$\begin{array}{l}\text { Urbanization } \\
\text { at main office }\end{array}$} \\
\hline Urban .......... & 65.1 & & 37.2 & 6.8 & 4.7 & 6.0 & 17.7 & 31.9 & 46.4 & 49.6 & & \\
\hline Rural & 63.3 & 36.6 & 37.4 & 6.3 & 6.1 & 4.4 & 14.9 & 23.0 & $\begin{array}{l}40.4 \\
47.8\end{array}$ & $\begin{array}{l}49.0 \\
42.6\end{array}$ & $\begin{array}{l}50.4 \\
66.6\end{array}$ & 92.8 \\
\hline \multirow{2}{*}{\multicolumn{13}{|c|}{ Number of offices }} \\
\hline One ............... & & & 35.9 & 5.7 & 4.2 & 5.3 & 15.6 & 30.3 & 46.9 & 46.4 & 58.0 & 92.1 \\
\hline Two ............ & 78.2 & 50.4 & 44.2 & 7.0 & 9.3 & 6.2 & 21.8 & 29.0 & 44.6 & 56.9 & 71.8 & 97.8 \\
\hline Three or more & 85.6 & 61.9 & 48.0 & 24.7 & 11.3 & 9.9 & 36.7 & 32.9 & 45.9 & 62.9 & 74.9 & 99.4 \\
\hline \multicolumn{13}{|l|}{$\begin{array}{l}\text { Sales area } \\
\text { Primarily within the }\end{array}$} \\
\hline United States .... & 64.1 & 38.2 & 36.9 & 6.7 & 4.8 & 5.7 & 16.7 & 29.7 & 46.3 & 48.2 & 60.4 & 92.9 \\
\hline International or global & 77.2 & 53.1 & 44.2 & 5.0 & 8.4 & 5.2 & 27.4 & 40.7 & 53.6 & 46.0 & 53.3 & 92.9 \\
\hline \multicolumn{13}{|l|}{ Management } \\
\hline By owner .... & 63.7 & 38.2 & 36.4 & 6.1 & 4.7 & 5.4 & 16.3 & 30.3 & 47.2 & 48.0 & 59.2 & 92.6 \\
\hline Hired ........ & 80.4 & 50.7 & 49.1 & 14.7 & 9.2 & 10.2 & 29.2 & 30.7 & 39.4 & 49.7 & 72.3 & 99.0 \\
\hline \multicolumn{13}{|l|}{$\begin{array}{l}\text { Race, ethnicity, and sex } \\
\text { of majority owners }\end{array}$} \\
\hline Nonwhite or Hispanic . & 62.3 & 35.7 & 38.1 & 3.9 & 4.8 & 4.7 & 10.5 & 36.6 & 39.9 & 46.7 & 48.6 & 90.6 \\
\hline Non-Hispanic white & 64.9 & 39.6 & 36.9 & 7.1 & 5.0 & 5.6 & 18.1 & 29.8 & 47.9 & 48.3 & 61.9 & 93.1 \\
\hline White $\ldots \ldots \ldots \ldots$ & 64.8 & 39.5 & 36.9 & 6.9 & 4.9 & 5.7 & 17.9 & 30.2 & 47.4 & 48.4 & 61.4 & 93.2 \\
\hline & 51.8 & 31.7 & 29.2 & $*$ & $*$ & * & * & 35.2 & 30.5 & 36.1 & 34.8 & 86.1 \\
\hline $\begin{array}{c}\text { Asian, Native Hawaiian, } \\
\text { or other Pacific }\end{array}$ & & & & & & & & & & & & \\
\hline & 71.2 & 37.7 & 51.2 & 5.0 & 7.7 & 3.9 & 10.5 & 31.6 & 48.0 & 53.4 & 56.4 & 92.5 \\
\hline $\begin{array}{l}\text { American Indian or } \\
\text { Alaska Native }\end{array}$ & 51.3 & 28.4 & 28.2 & * & * & * & * & * & 51.4 & 39.7 & 51.4 & 87.4 \\
\hline $\begin{array}{l}\text { Hispanic .......... } \\
\text { Non-Hispanic }\end{array}$ & $\begin{array}{l}64.7 \\
64.6\end{array}$ & $\begin{array}{l}39.1 \\
38.9\end{array}$ & $\begin{array}{l}34.8 \\
37.2\end{array}$ & $\begin{array}{l}* \\
6.9\end{array}$ & $\stackrel{*}{5.1}$ & $\begin{array}{l}8.0 \\
5.5\end{array}$ & $\begin{array}{l}14.6 \\
17.2\end{array}$ & $\begin{array}{l}39.0 \\
30.0\end{array}$ & $\begin{array}{l}35.3 \\
47.2\end{array}$ & $\begin{array}{l}50.5 \\
48.0\end{array}$ & $\begin{array}{l}49.1 \\
60.5\end{array}$ & $\begin{array}{l}92.3 \\
93.0\end{array}$ \\
\hline Female ........ & 61.6 & 38.6 & 36.0 & 6.3 & 2.7 & 3.6 & 10.2 & 35.9 & 49.2 & 42.9 & 46.9 & 88.2 \\
\hline Male .................. & 64.6 & 38.4 & 36.2 & 6.9 & 6.0 & 6.6 & 19.9 & 29.8 & 45.1 & 49.8 & 64.0 & 93.8 \\
\hline $\begin{array}{r}\text { Ownership divided } \\
\text { equally by sex }\end{array}$ & 69.9 & 41.8 & 43.7 & 5.7 & 3.6 & 4.6 & 14.7 & 26.6 & 50.6 & 48.5 & 62.9 & 96.9 \\
\hline
\end{tabular}

Note: Refer to notes to table 6.A.

n.a. Not available.

. . Not applicable.

"any service" (96 percent) nearly matches the reported use of "any liquid asset account."

For business savings accounts, firm size seems to play a significant role in usage, with the smallest firms the least likely to have savings accounts. Less than one-fifth of firms with four or fewer workers had savings accounts, compared with more than one-third of firms with twenty or more workers. By organizational form, corporations were the most likely type of firm to have a savings account. Firms owned by non-Hispanic whites were more likely than nonwhite or Hispanic firms to have a savings account, and male-owned businesses or businesses owned equally by males and females were more likely than were female-owned firms to have a business savings account. 


\section{Credit Lines, Loans, and Capital Leases}

More than 60 percent of small businesses reported outstanding credit in the form of a credit line, a loan, or a capital lease (table 6.A). Overall, the incidence of credit lines, loans, and capital leases increased from 55 percent to more than 60 percent between 1998 and 2003.

As in 1998, the most widely used types of credit in 2003 were credit lines and vehicle loans. ${ }^{21}$ The importance of these two credit types seems to be growing. The share of firms with lines increased from 28 percent to 34 percent, and the share with vehicle loans rose from 21 percent to 26 percent. The incidences of mortgages, equipment loans, and "other" loans were similar to their 1998 levels; leases declined somewhat. ${ }^{22}$ The increase in the percentage of firms with lines may be due in part to commercial banks' increased use of credit-scoring models for that type of loan. ${ }^{23}$ Alternatively, the increase may have been due to differences in the economic environment. For example, 2002 and 2003 were years of historically low interest rates. The low rates may have stimulated increased loan demand among small businesses.

The incidence of lines, loans, and leases increased with firm size. More than 90 percent of the largest firms (100-499 employees or at least $\$ 5$ million in sales) had one of these types of credit in 2003, compared with fewer than 50 percent of the firms with 1 employee or with less than $\$ 100,000$ in sales. Corporations were more likely than other types of firms to have had outstanding loans in 2003. Firms in services (business or professional) had fewer outstanding loans than those in other industries, perhaps because they require less inventory and equipment.

Credit incidence did not appear to vary systematically with the age of the business, even though

21. In this article, use of a credit line refers to the availability of a credit line and not necessarily to the borrowing of funds from the line. Survey information on outstanding credit-line balances will be available at a later date.

22. The majority of "other" loans were loans that could not be classified as credit lines, mortgages, equipment loans, vehicle loans, or capital leases. Such loans were most likely term loans, and roughly 70 percent of them were unsecured.

23. Although statistics on the use of credit scoring by commercial banks are somewhat dated (W. Scott Frame, Aruna Srinavasan, and Lynn Woosley, 2001, "The Effect of Credit Scoring on Small Business Lending," Journal of Money, Credit, and Banking, vol. 33, August, pp. 813-25), some indirect evidence is available from data gathered under the Community Reinvestment Act. These data indicate that between 1998 and 2003, the growth in the number of small business loans has been far greater than growth in the dollar amount of small business loans, which suggests that much of the growth in business loans has been in smaller loans. Small lines of credit are likely to be one of the types of credit most amenable to credit scoring. depository institutions typically require borrowers to have several years of financial history to qualify for credit. The youngest firms (those under current ownership fewer than five years) reported nearly the same incidence of borrowing as did more mature firms. However, a somewhat different picture emerges when specific types of credit are examined. The youngest firms were least likely to have outstanding lines, vehicle loans, and equipment loans. They were also the most likely to have had leases and "other" loans. It may be that the hypothesis regarding young firms and depository institutions does not apply equally to all loan types, or that other factors, such as personal relationships with financial institutions, may offset to some degree the lack of information available for younger firms.

The incidence of lines, loans, and leases also varied somewhat with owner characteristics, such as race, ethnicity, and sex. In 2003, 61 percent of whiteowned businesses had outstanding credit. In contrast, about 48 percent of either black-owned or femaleowned businesses and 52 percent of Asian-owned firms had outstanding credit. Firms owned equally by men and women appear to be most similar to maleowned businesses in their reported use of lines, loans, and leases.

Incidence also varied by credit type for these firm types. For each credit type, female-owned businesses were less apt to have credit than were male-owned firms. Black-owned firms were less likely than whiteowned firms to have had lines of credit, mortgages, and equipment loans. Black-owned firms had about the same incidence of vehicle loans and capital leases but reported a higher incidence of "other" loans.

Non-Hispanic-owned firms and Hispanic-owned firms were equally likely to have outstanding credit, although the incidence varied by type of credit. Non-Hispanic firms were more likely than Hispanicowned firms to have equipment loans and leases, whereas Hispanic-owned firms were more likely to have vehicle and "other" loans. Some of the differences by owner race, ethnicity, and sex may be attributable to differences in firm characteristics, such as size. ${ }^{24}$

24. Research on this topic using multivariate analysis is available in, for example, Ken S. Cavalluzzo, Linda C. Cavalluzzo, and John D. Wolken (2002), "Competition, Small Business Financing, and Discrimination: Evidence from a New Survey," Journal of Business, vol. 75 (October), pp. 641-79; and Ken S. Cavalluzzo and John D. Wolken (2005), "Small Business Loan Turndowns, Personal Wealth, and Discrimination," Journal of Business, vol. 78 (November), pp. 2153-77. 


\section{Financial Management Services}

In the 2003 survey, financial management services covered six broad categories of service, one more than in previous surveys. The 2003 categories were transaction services, credit card and debit card processing, cash management, credit-related services, brokerage services, and trust and pension services. ${ }^{25}$ Nearly two-thirds of firms used at least one financial management service in 2003, compared with one-half of firms in 1998 (table 6.B). Part of the increase is likely due to the difference between the two surveys in the wording of the questions. In 1998, transaction services included credit card (but not debit card) processing. In 2003, credit card processing was removed from transaction services and added to a new service category that consisted of credit card and debit card processing. Consequently, direct comparisons of "any" financial management service or of transaction services with earlier surveys is difficult. ${ }^{26}$ In particular, the decline in the use of transaction services, from 41 percent to 39 percent, probably reflects, at least in part, the change in the definition of that category.

\section{Overall Results}

The evidence generally points to a growing importance for financial management services. An increase in incidence was recorded for each of the financial management services whose definitions were constant across surveys: for cash management services, from 5 percent to 7 percent; for credit-related services, from 3 percent to 5 percent; for brokerage services, from 4 percent to 6 percent; and for trust and pension services, from 13 percent to 17 percent.

The most widely used financial management service in 2003 continued to be transaction services

25. These categories cover the following specific servicesTransaction services: the provision of paper money and coins, depositing or clearing checks or drafts from business customers, the collection of night deposits, and wire transfers. Credit card and debit card processing services: the processing of credit card receipts, of signature-based debit (check-card) transactions, and of PIN-based debit transactions (credit card processing was previously combined with transaction services but was asked about separately in the 2003 survey). Cash management services: the provision of sweep accounts, zero-balance accounts, lockbox services, and other services designed to automatically invest liquid funds in liquid, interest-bearing assets. Credit-related services: the provision of banker's acceptances, letters of credit, and factoring. Trust and pension services: the provision of 401(k) plans, pension funds, and business trusts. Brokerage services: brokering the purchase and sale of stocks, bonds, and other securities.

26. A comparison of the services in the 1998 transactions services category to similar services from the 2003 survey is discussed later, in the section "Transaction and Card Processing Services."
(39 percent) followed closely by credit and debit card processing (37 percent). Trust and pension services were used by nearly 20 percent of firms, whereas cash management, credit-related, and brokerage services were each used by roughly 5 percent of firms. As was true for liquid asset services and for lines, loans, and leases, the use of financial management services increased with firm size. For the smallest firms (as measured by employment) just over 40 percent of firms used any financial management service, 25 percent used transaction services, 16 percent used card processing, and only a very small portion used other financial management services. In contrast, 96 percent of the largest firms (100-499 employees) used at least one financial management service; the most common was transaction services ( 70 percent), followed closely by trust and pension services (68 percent). Cash management and card processing services were used by 50 percent of the largest firms.

The proportion of proprietorships that used financial management services (52 percent) was smaller than that of firms with other organizational forms (70-77 percent); proprietorships may not need these services as much because they tend to be small and more likely than other types of firms to commingle personal and business accounts.

Firms differed in their use of financial management services by race, with black-owned firms and firms owned by American Indians or Alaska Natives somewhat less likely to have used financial management services than white non-Hispanic-owned firms. Female-owned firms were somewhat less likely to have used credit-related, brokerage, and trust and pension services than male-owned firms. Hispanicowned and Asian-owned firms used one or more financial-management services with about the same frequency as firms owned by non-Hispanic whites. However, differences in use exist among these groups for specific financial management categories. For example, Asian-owned firms were more likely to have used card processing and credit-related services than were white-owned firms. Hispanic-owned firms were less likely to have used trust and pension services than were non-Hispanic-owned firms. These differences could be related to firm size and industry classification.

\section{Transaction and Card Processing Services}

Although the collection of information on transaction services and card processing changed in 2003, the data exist to measure whether a firm used a transaction service or a card processor. In the 2003 survey, 
7. Use of transaction services and credit card processing by small businesses, by number of employees, 1998 and 2003 surveys

Percent

\begin{tabular}{|c|c|c|c|c|}
\hline \multirow[b]{2}{*}{ Number of employees } & \multicolumn{3}{|c|}{2003} & \multirow[b]{2}{*}{$\begin{array}{c}1998 \\
\text { transaction } \\
\text { service }\end{array}$} \\
\hline & $\begin{array}{c}\text { Transaction } \\
\text { service }\end{array}$ & $\begin{array}{l}\text { Credit card } \\
\text { processing }\end{array}$ & $\begin{array}{l}\text { Transaction } \\
\text { service and } \\
\text { credit card } \\
\text { processing }\end{array}$ & \\
\hline All firms $\ldots \ldots \ldots \ldots$ & 38.9 & 35.6 & 56.2 & 41.1 \\
\hline $\begin{array}{l}0-1 \\
2-4 \\
5-19 \ldots \ldots \ldots \ldots \ldots \ldots \\
20-49 \ldots \ldots \ldots \ldots \ldots \\
50-99 \ldots \ldots \ldots \ldots \ldots \\
100-499 \ldots \ldots \ldots \ldots \ldots \\
\end{array}$ & $\begin{array}{l}25.2 \\
34.9 \\
46.8 \\
61.0 \\
61.3 \\
70.3\end{array}$ & $\begin{array}{l}14.7 \\
32.1 \\
49.0 \\
55.4 \\
50.8 \\
49.4\end{array}$ & $\begin{array}{l}34.3 \\
51.9 \\
69.9 \\
80.8 \\
76.2 \\
79.8\end{array}$ & $\begin{array}{l}28.1 \\
35.7 \\
53.3 \\
59.2 \\
56.8 \\
70.3\end{array}$ \\
\hline
\end{tabular}

Note: In the 2003 survey, credit card processing was separate from transaction services; in the 1998 survey, transaction services included credit card processing. For further details, refer to text.

respondents who reported using a credit or debit card processor were asked to identify which services they received. The responses make it possible to separate firms that used credit card processing from those that used only debit card processing. The data indicate that if the 2003 question were asked exactly as it had been in 1998, growth in transaction services would have been observed. In 2003, 56 percent of all firms reported using transaction services or credit card processing (a category of service comparable to that asked in 1998), up from the 41 percent of firms who reported doing so in 1998 (table 7). The use of transaction services increased with firm size, with more than three-fourths of the largest firms using them in 2003.
Among small businesses, 37 percent used a card processing service (table 8). The majority of these businesses used a credit card processor (96 percent). The usage pattern varied across firm industry. For example, 72 percent of retail trade firms used some type of card processor, but only 9 percent of insurance agents and real estate firms did so. The pattern by type of processor used, however, was the same for all types of firms; credit card processors were always the most common, followed by processors for signature debit card and PIN debit card transactions. Usage also increased with the size of the firm: 16 percent of the very smallest firms (0-1 employees) used credit or debit card services or both, compared with 50 percent of the largest firms (100-499 employees).

\section{Owner Loans, Credit Cards, and Trade Credit}

In addition to using credit lines, loans, and capital leases, many small businesses obtain financing by borrowing from the firm's owners (owner loans), borrowing via credit cards, or borrowing from suppliers of goods and services (trade credit).

These alternative forms of credit are different from lines, loans, and leases in a number of ways. For example, owner loans are clearly not arm's-length transactions. In the case of credit cards, the interest rates charged may exceed the interest rates for other types of loans; moreover, credit cards, unlike typical loans, provide a convenient means of paying bills and tracking expenses. Trade credit is generally used in connection with the purchase of goods and services

8. Use of credit and debit card processing services by small businesses and specific type and number of services used, by number of employees and industry, 2003 survey

Percent except as noted

\begin{tabular}{|c|c|c|c|c|c|}
\hline \multirow{3}{*}{ Characteristic } & \multirow{3}{*}{$\begin{array}{l}\text { Any card } \\
\text { processing } \\
\text { service }\end{array}$} & \multicolumn{4}{|c|}{$\begin{array}{l}\text { Type and median number of services used among users } \\
\text { of card processing services }\end{array}$} \\
\hline & & \multirow{2}{*}{$\begin{array}{l}\text { Credit card } \\
\text { processing }\end{array}$} & \multicolumn{2}{|c|}{$\begin{array}{l}\text { Debit card processing, } \\
\text { by type of transaction }\end{array}$} & \multirow{2}{*}{$\begin{array}{l}\text { Median } \\
\text { number used } \\
\text { (of three) }\end{array}$} \\
\hline & & & Signature & $\mathrm{PIN}^{1}$ & \\
\hline 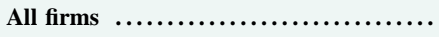 & 37.2 & 95.6 & 48.8 & 26.6 & 2 \\
\hline 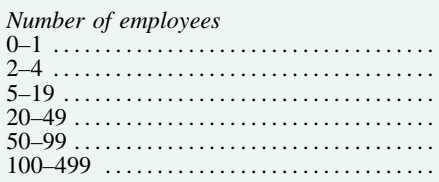 & $\begin{array}{l}16.1 \\
33.7 \\
51.1 \\
56.3 \\
51.5 \\
50.1\end{array}$ & $\begin{array}{l}91.1 \\
95.3 \\
95.8 \\
98.4 \\
98.7 \\
98.6\end{array}$ & $\begin{array}{l}51.7 \\
49.4 \\
48.9 \\
49.2 \\
33.1 \\
34.4\end{array}$ & $\begin{array}{l}17.1 \\
25.8 \\
30.8 \\
22.3 \\
21.9 \\
19.0\end{array}$ & $\begin{array}{l}1 \\
2 \\
2 \\
2 \\
1 \\
1\end{array}$ \\
\hline 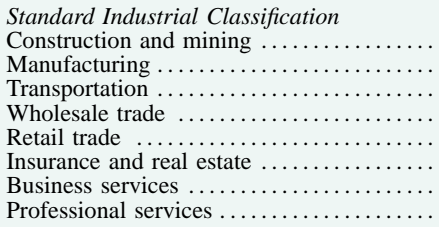 & $\begin{array}{r}13.9 \\
36.5 \\
37.4 \\
48.2 \\
72.1 \\
9.3 \\
38.5 \\
24.8\end{array}$ & $\begin{array}{r}86.5 \\
94.8 \\
81.8 \\
100.0 \\
96.8 \\
81.9 \\
98.2 \\
93.7\end{array}$ & $\begin{array}{l}24.9 \\
31.6 \\
43.2 \\
26.2 \\
61.8 \\
42.5 \\
47.3 \\
49.1\end{array}$ & $\begin{array}{l}18.8 \\
16.8 \\
20.7 \\
19.3 \\
33.7 \\
22.1 \\
28.3 \\
18.8\end{array}$ & $\begin{array}{l}1 \\
1 \\
1 \\
1 \\
2 \\
1 \\
2\end{array}$ \\
\hline
\end{tabular}

1. PIN Personal identification number 
from a specific supplier, whereas funds from lines, loans, and leases are often available for general purposes and are not restricted to purchases from a single supplier. Also, when outstanding trade credit balances are not repaid in a relatively short period, the finance charges may exceed those on other loans.

\section{Loans from Owners}

Of the small businesses that could have received loans from owners (that is, those that were organized as corporations or partnerships), the proportion with such loans rose slightly between the 1998 and 2003 surveys, from 28 percent to 30 percent (table 6.B).

Because they generally have fewer credit options, smaller firms might seem more likely than larger firms to borrow from their owners. This was not the case in 2003. The incidence of owner loans differed across size groups with no specific pattern except that the smallest size groups (0-1 employee, less than $\$ 25,000$ in sales, or less than $\$ 25,000$ in assets) were the least likely to have reported owner loans. Only about one-fourth of the smallest firms reported owner loans, versus 30 percent for all small businesses.

\section{Credit Cards}

Small businesses were somewhat more likely to have used credit cards in 2003 than in 1998. The percentage that used personal credit cards (47 percent in 2003) remained about the same, but the percentage that used business credit cards increased from 34 percent to 48 percent.

Credit cards are a convenient means of making payments and tracking expenses. Anecdotal evidence suggests that many smaller and newer businesses also use credit cards as a source of credit, even though they may have higher interest rates than other forms of credit. Lenders sometimes ration credit to high-risk firms. Thus, firms just starting out and those having little credit history may be perceived as high risk and may therefore rely on credit cards as a substitute for other types of loans. The descriptive statistics on the use of credit cards are only somewhat consistent with this hypothesis. Personal credit card use was highest among the smallest firms, averaging about 50 percent. But even among the largest firms, about 33 percent reported using personal credit cards for business expenses. In contrast, the use of business credit cards generally increased with firm size-roughly one-third of the smallest firms used them, compared with about three-fifths of the larger firms.

The use of personal credit cards did decline somewhat with firm age, but the use of business credit cards did not appear to be related to age. Proprietorships were the organizational form most likely to have used personal credit cards and the least likely to have used business cards, but proprietorships are also generally smaller than other organizational forms. Use of credit cards did not vary much by industrytransportation firms were the least likely to use personal credit cards, and insurance and real estate firms and retail trade firms the least likely to use business credit cards. By owner characteristic, non-Hispanic white-owned businesses were more likely to use personal credit cards and just about as likely to use business credit cards as Hispanic-owned or nonwhiteowned businesses. Relative to male-owned firms, female-owned firms were more likely to use personal credit cards and less likely to use business credit cards.

As indicated earlier, some firms may use credit cards simply for the convenience of making payments and tracking expenses. In 2003, 73 percent of personal credit card users and 71 percent of business credit card users reported that they paid their statement balance in full by the payment-due date. For these businesses, credit cards appear to be used primarily for transactional convenience.

\section{Trade Credit}

A firm receives trade credit when its suppliers collect payment after, rather than at the time of, the receipt of goods or services. Most trade credit is extended to firms for a very short period (thirty or sixty days) and is always granted in connection with specific purchases. Businesses use trade credit to reduce transaction costs and sometimes as a form of financing. Allowing available funds to be used for other purposes is one way that trade credit reduces the transaction cost that businesses would incur if they had to make payment at the time of receipt. If the firm does not pay the bill for the goods or services on time, trade credit becomes a form of financing. Because the interest rates charged on overdue balances can be quite high, it is reasonable to expect that the firms using trade credit for longer-term financing purposes would have had difficulty obtaining credit from other sources. ${ }^{27}$

Trade credit was used by 60 percent of small businesses in 2003, an incidence of use that exceeds that for all other financial services except checking. In

27. Firms were asked to report on the monthly penalty they would be charged if they paid after the due date. The average rate reported was a little more than 1 percent. The median, 75th percentile, and 90th percentile were 1 percent, 1.5 percent, and 2 percent respectively. 
9. Use of selected suppliers of financial services by small businesses, by selected characteristics of firms, 2003 survey Percent

\begin{tabular}{|c|c|c|c|c|c|c|c|c|c|c|c|c|c|c|c|c|}
\hline \multirow[b]{3}{*}{ Characteristic } & \multirow[b]{3}{*}{$\begin{array}{c}\text { Any } \\
\text { supplier }\end{array}$} & \multirow{2}{*}{\multicolumn{4}{|c|}{ Depository }} & \multicolumn{11}{|c|}{ Nondepository } \\
\hline & & & & & & \multirow[b]{2}{*}{ Any } & \multicolumn{5}{|c|}{ Primarily financial } & \multicolumn{5}{|c|}{ Other nondepository } \\
\hline & & Any & $\begin{array}{c}\text { Com- } \\
\text { mercial } \\
\text { bank }\end{array}$ & $\begin{array}{c}\text { Thrift } \\
\text { institu- } \\
\text { tion }^{1}\end{array}$ & $\begin{array}{l}\text { Credit } \\
\text { union }\end{array}$ & & Any & $\begin{array}{c}\text { Finance } \\
\text { com- } \\
\text { pany or } \\
\text { factor }\end{array}$ & $\begin{array}{c}\text { Broker- } \\
\text { age or } \\
\text { pension } \\
\text { firm }\end{array}$ & $\begin{array}{c}\text { Leasing } \\
\text { com- } \\
\text { pany }\end{array}$ & $\begin{array}{c}\text { Insur- } \\
\text { ance or } \\
\text { mort- } \\
\text { gage } \\
\text { com- } \\
\text { pany }\end{array}$ & Any & $\begin{array}{c}\text { Card or } \\
\text { check } \\
\text { pro- } \\
\text { cessing } \\
\text { firm }^{2}\end{array}$ & $\begin{array}{c}\text { Govern- } \\
\text { ment }\end{array}$ & $\begin{array}{c}\text { Family } \\
\text { and in- } \\
\text { divid- } \\
\text { uals }\end{array}$ & Other $^{3}$ \\
\hline $\begin{array}{l}\text { All firms } \\
2003 \ldots \ldots \ldots \ldots \ldots \ldots \\
1998 \ldots \ldots \ldots \ldots \ldots \ldots\end{array}$ & $\begin{array}{l}96.4 \\
96.1\end{array}$ & $\begin{array}{l}95.9 \\
95.2\end{array}$ & $\begin{array}{l}86.5 \\
87.3\end{array}$ & $\begin{array}{r}13.8 \\
9.2\end{array}$ & $\begin{array}{l}8.1 \\
5.9\end{array}$ & $\begin{array}{l}54.1 \\
39.9\end{array}$ & $\begin{array}{l}40.3 \\
31.0\end{array}$ & $\begin{array}{l}25.2 \\
15.5\end{array}$ & $\begin{array}{l}14.9 \\
11.3\end{array}$ & $\begin{array}{l}4.5 \\
7.0\end{array}$ & $\begin{array}{l}5.4 \\
3.7\end{array}$ & $\begin{array}{l}24.3 \\
15.6\end{array}$ & $\begin{array}{r}13.4 \\
4.0\end{array}$ & $\begin{array}{l}1.2 \\
1.0\end{array}$ & $\begin{array}{l}6.6 \\
6.2\end{array}$ & $\begin{array}{l}5.5 \\
5.6\end{array}$ \\
\hline 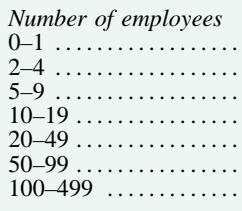 & $\begin{array}{r}88.9 \\
96.9 \\
99.8 \\
100.0 \\
100.0 \\
100.0 \\
100.0\end{array}$ & $\begin{array}{r}87.2 \\
96.4 \\
99.5 \\
100.0 \\
100.0 \\
99.9 \\
100.0\end{array}$ & $\begin{array}{l}71.6 \\
86.2 \\
92.8 \\
95.1 \\
97.8 \\
98.7 \\
96.8\end{array}$ & $\begin{array}{r}14.2 \\
13.9 \\
13.5 \\
14.2 \\
13.0 \\
9.2 \\
17.0\end{array}$ & $\begin{array}{l}9.6 \\
9.2 \\
6.6 \\
7.1 \\
4.4 \\
* \\
*\end{array}$ & $\begin{array}{l}35.6 \\
47.1 \\
66.9 \\
71.4 \\
75.9 \\
86.4 \\
82.6\end{array}$ & $\begin{array}{l}25.7 \\
33.4 \\
48.5 \\
57.3 \\
62.6 \\
78.0 \\
75.6\end{array}$ & $\begin{array}{l}16.4 \\
20.8 \\
30.8 \\
34.5 \\
40.3 \\
46.0 \\
42.1\end{array}$ & $\begin{array}{r}8.1 \\
10.6 \\
18.2 \\
25.2 \\
27.5 \\
36.4 \\
40.9\end{array}$ & $\begin{array}{r}* \\
3.0 \\
6.2 \\
7.5 \\
7.0 \\
13.3 \\
14.1\end{array}$ & $\begin{array}{r}3.0 \\
4.2 \\
5.3 \\
6.1 \\
14.9 \\
18.6 \\
16.7\end{array}$ & $\begin{array}{l}14.7 \\
21.2 \\
33.2 \\
32.3 \\
30.5 \\
33.0 \\
30.7\end{array}$ & $\begin{array}{r}6.8 \\
12.5 \\
18.5 \\
18.2 \\
16.3 \\
14.2 \\
11.6\end{array}$ & $\begin{array}{c}* \\
* \\
* \\
* \\
3.4 \\
* \\
4.8\end{array}$ & $\begin{array}{r}4.0 \\
5.5 \\
9.2 \\
10.2 \\
7.7 \\
4.6 \\
10.9\end{array}$ & $\begin{array}{r}3.1 \\
4.3 \\
7.0 \\
8.6 \\
7.2 \\
14.8 \\
11.7\end{array}$ \\
\hline 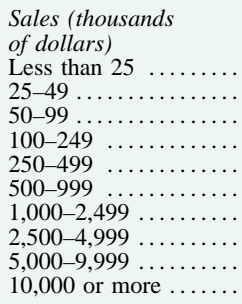 & $\begin{array}{r}81.4 \\
97.2 \\
97.6 \\
98.9 \\
99.6 \\
100.0 \\
100.0 \\
100.0 \\
100.0 \\
100.0\end{array}$ & $\begin{array}{r}80.0 \\
96.1 \\
96.0 \\
98.9 \\
99.4 \\
99.8 \\
99.6 \\
100.0 \\
99.9 \\
100.0\end{array}$ & $\begin{array}{l}65.5 \\
78.0 \\
84.9 \\
89.8 \\
91.8 \\
94.6 \\
94.4 \\
98.4 \\
99.4 \\
96.3\end{array}$ & $\begin{array}{l}12.3 \\
19.3 \\
13.2 \\
13.0 \\
14.7 \\
12.4 \\
15.4 \\
10.4 \\
10.7 \\
11.8\end{array}$ & $\begin{array}{r}9.0 \\
14.0 \\
9.6 \\
8.1 \\
7.0 \\
6.4 \\
5.4 \\
* \\
* \\
*\end{array}$ & $\begin{array}{l}24.7 \\
42.7 \\
43.8 \\
49.4 \\
63.8 \\
72.0 \\
71.8 \\
77.3 \\
89.0 \\
84.1\end{array}$ & $\begin{array}{l}13.7 \\
28.4 \\
28.7 \\
34.9 \\
45.8 \\
57.0 \\
60.7 \\
68.3 \\
79.9 \\
79.7\end{array}$ & $\begin{array}{r}9.2 \\
17.5 \\
17.7 \\
22.1 \\
26.8 \\
38.4 \\
34.5 \\
46.9 \\
55.5 \\
42.6\end{array}$ & $\begin{array}{r}4.4 \\
9.9 \\
10.3 \\
9.0 \\
16.1 \\
17.4 \\
29.7 \\
31.3 \\
41.2 \\
48.8\end{array}$ & $\begin{array}{c}* \\
* \\
* \\
4.3 \\
5.5 \\
7.4 \\
8.5 \\
5.0 \\
8.1 \\
14.6\end{array}$ & $\begin{array}{c}* \\
* \\
* \\
4.1 \\
7.1 \\
5.9 \\
7.4 \\
14.4 \\
13.4 \\
17.2\end{array}$ & $\begin{array}{l}13.5 \\
19.8 \\
18.6 \\
22.8 \\
29.1 \\
36.2 \\
29.7 \\
27.3 \\
27.5 \\
30.8\end{array}$ & $\begin{array}{r}6.7 \\
10.4 \\
9.4 \\
13.4 \\
16.9 \\
21.0 \\
18.3 \\
11.8 \\
9.0 \\
13.0\end{array}$ & $\begin{array}{c}* \\
* \\
* \\
* \\
* \\
* \\
* \\
4.5 \\
* \\
3.0\end{array}$ & $\begin{array}{l}5.2 \\
6.4 \\
5.3 \\
6.7 \\
8.4 \\
8.6 \\
5.0 \\
7.5 \\
6.1 \\
7.2\end{array}$ & $\begin{array}{r}* \\
* \\
* \\
3.4 \\
6.2 \\
8.2 \\
9.9 \\
8.6 \\
13.8 \\
12.9\end{array}$ \\
\hline $\begin{array}{l}\text { Assets (thousands } \\
\text { of dollars) } \\
\text { Less than } 25 \ldots \ldots \ldots \\
25-49 \ldots \ldots \ldots \ldots \ldots \\
50-99 \ldots \ldots \ldots \ldots \ldots \\
100-249 \ldots \ldots \ldots \ldots \ldots \\
250-499 \ldots \ldots \ldots \ldots \ldots \\
500-999 \ldots \ldots \ldots \ldots \\
1,000-2,499 \ldots \ldots \ldots \ldots \\
2,500-4,999 \ldots \ldots \ldots \ldots \\
5,000 \text { or more } \ldots \ldots \ldots\end{array}$ & $\begin{array}{r}90.0 \\
98.2 \\
99.8 \\
99.1 \\
99.7 \\
100.0 \\
100.0 \\
100.0 \\
100.0\end{array}$ & $\begin{array}{r}89.0 \\
97.7 \\
99.4 \\
98.7 \\
99.3 \\
100.0 \\
100.0 \\
100.0 \\
98.5\end{array}$ & $\begin{array}{l}73.0 \\
89.6 \\
90.5 \\
92.6 \\
94.2 \\
95.5 \\
95.2 \\
94.9 \\
97.8\end{array}$ & $\begin{array}{l}13.7 \\
15.9 \\
11.0 \\
11.4 \\
15.2 \\
16.4 \\
15.4 \\
14.0 \\
19.1\end{array}$ & $\begin{array}{r}8.9 \\
6.9 \\
12.7 \\
6.6 \\
6.2 \\
9.1 \\
5.3 \\
* \\
*\end{array}$ & $\begin{array}{l}35.7 \\
43.5 \\
59.8 \\
62.6 \\
71.0 \\
66.3 \\
79.3 \\
79.6 \\
74.9\end{array}$ & $\begin{array}{l}23.7 \\
30.0 \\
41.9 \\
44.8 \\
55.0 \\
56.6 \\
69.4 \\
74.1 \\
71.1\end{array}$ & $\begin{array}{l}15.5 \\
20.3 \\
27.1 \\
26.2 \\
32.2 \\
34.5 \\
44.3 \\
48.2 \\
39.3\end{array}$ & $\begin{array}{r}6.5 \\
10.1 \\
14.3 \\
17.2 \\
19.9 \\
22.3 \\
33.7 \\
32.9 \\
40.3\end{array}$ & $\begin{array}{r}2.2 \\
* \\
3.8 \\
5.3 \\
10.2 \\
5.4 \\
8.6 \\
10.1 \\
7.8\end{array}$ & $\begin{array}{c}2.2 \\
* \\
6.1 \\
5.5 \\
5.3 \\
8.9 \\
13.1 \\
20.4 \\
16.5\end{array}$ & $\begin{array}{l}16.9 \\
18.3 \\
26.8 \\
29.8 \\
34.5 \\
32.4 \\
27.8 \\
30.0 \\
21.7\end{array}$ & $\begin{array}{r}8.0 \\
12.3 \\
15.6 \\
19.9 \\
19.8 \\
15.4 \\
11.0 \\
10.6 \\
8.5\end{array}$ & $\begin{array}{l}* \\
* \\
* \\
* \\
* \\
* \\
* \\
* \\
2.4\end{array}$ & $\begin{array}{r}5.1 \\
* \\
9.5 \\
5.0 \\
9.3 \\
12.1 \\
7.8 \\
6.1 \\
5.7\end{array}$ & $\begin{array}{r}3.6 \\
* \\
4.4 \\
6.3 \\
8.2 \\
8.4 \\
10.4 \\
11.8 \\
9.6\end{array}$ \\
\hline $\begin{array}{l}\text { Organizational form } \\
\text { Proprietorship } \ldots \ldots \ldots \\
\text { Partnership ............. } \\
\text { S corporation ......... } \\
\text { C corporation } \ldots \ldots \ldots\end{array}$ & $\begin{array}{l}93.0 \\
97.3 \\
99.5 \\
99.6\end{array}$ & $\begin{array}{l}92.0 \\
96.9 \\
99.2 \\
99.4\end{array}$ & $\begin{array}{l}78.5 \\
89.6 \\
93.2 \\
94.1\end{array}$ & $\begin{array}{l}15.9 \\
14.0 \\
12.5 \\
10.4\end{array}$ & $\begin{array}{r}11.1 \\
9.1 \\
5.0 \\
5.3\end{array}$ & $\begin{array}{l}44.3 \\
49.8 \\
64.0 \\
64.3\end{array}$ & $\begin{array}{l}31.3 \\
35.1 \\
48.9 \\
51.7\end{array}$ & $\begin{array}{l}20.1 \\
20.3 \\
30.8 \\
30.9\end{array}$ & $\begin{array}{r}9.8 \\
13.1 \\
18.3 \\
23.6\end{array}$ & $\begin{array}{l}2.2 \\
4.4 \\
7.3 \\
5.4\end{array}$ & $\begin{array}{l}4.4 \\
4.9 \\
5.5 \\
8.2\end{array}$ & $\begin{array}{l}19.8 \\
22.4 \\
29.9 \\
27.1\end{array}$ & $\begin{array}{l}10.3 \\
11.9 \\
17.6 \\
14.6\end{array}$ & $\begin{array}{c}* \\
* \\
2.3 \\
1.4\end{array}$ & $\begin{array}{l}6.8 \\
* \\
7.4 \\
6.1\end{array}$ & $\begin{array}{l}3.8 \\
7.1 \\
6.5 \\
7.1\end{array}$ \\
\hline $\begin{array}{l}\text { Standard Industrial } \\
\text { Classification } \\
\text { Construction and } \\
\text { mining ............ }\end{array}$ & & & & & 9.1 & & & & & & & & & & & \\
\hline & 97.7 & $\begin{array}{l}90.2 \\
97.7\end{array}$ & $\begin{array}{l}80.1 \\
90.6 \\
909\end{array}$ & $\begin{array}{l}11.3 \\
11.6\end{array}$ & 8.1 & & & $\begin{array}{l}34.3 \\
24.5\end{array}$ & $\begin{array}{l}10.0 \\
16.8\end{array}$ & $\begin{array}{l}4.8 \\
6.1\end{array}$ & 4.7 & $\begin{array}{l}14.8 \\
30.6\end{array}$ & 13.5 & 2.4 & $\begin{array}{r}3.4 \\
10.6\end{array}$ & \\
\hline $\begin{array}{l}\text { Transportation .... } \\
\text { Wholesale trade }\end{array}$ & $\begin{array}{l}99.5 \\
98.8\end{array}$ & $\begin{array}{l}99.5 \\
97.7\end{array}$ & $\begin{array}{l}90.9 \\
92.0\end{array}$ & $\begin{array}{l}10.0 \\
10.5\end{array}$ & $\begin{array}{c}15.7 \\
*\end{array}$ & $\begin{array}{l}61.9 \\
62.7\end{array}$ & $\begin{array}{l}47.5 \\
47.8\end{array}$ & $\begin{array}{l}38.4 \\
31.0\end{array}$ & $\begin{array}{r}6.3 \\
21.7\end{array}$ & $\begin{array}{l}6.6 \\
3.2\end{array}$ & $\begin{array}{l}5.8 \\
5.8\end{array}$ & $\begin{array}{l}27.1 \\
27.7\end{array}$ & $\begin{array}{l}11.0 \\
17.8\end{array}$ & $\begin{array}{l}* \\
*\end{array}$ & $\begin{array}{r}13.1 \\
5.2\end{array}$ & $\begin{array}{l}6.7 \\
6.8\end{array}$ \\
\hline $\begin{array}{l}\text { Retail trade } \ldots \ldots \ldots \\
\text { Insurance and }\end{array}$ & 97.4 & 97.1 & 88.4 & 15.7 & 5.6 & 59.4 & 31.9 & 22.3 & 8.7 & 4.3 & 4.9 & 39.8 & 28.3 & 1.9 & 7.8 & 6.1 \\
\hline $\begin{array}{r}\text { real estate ........ } \\
\text { Business services ..... } \\
\text { Professional services .. }\end{array}$ & $\begin{array}{l}96.3 \\
94.9 \\
95.9\end{array}$ & $\begin{array}{l}96.0 \\
94.0 \\
95.1\end{array}$ & $\begin{array}{l}90.2 \\
82.0 \\
85.0\end{array}$ & $\begin{array}{l}18.3 \\
14.2 \\
12.7\end{array}$ & $\begin{array}{l}7.8 \\
9.6 \\
7.4\end{array}$ & $\begin{array}{l}46.4 \\
49.9 \\
53.0\end{array}$ & $\begin{array}{l}43.3 \\
35.3 \\
46.0\end{array}$ & $\begin{array}{l}19.3 \\
23.4 \\
22.9\end{array}$ & $\begin{array}{l}21.2 \\
11.3 \\
24.3\end{array}$ & $\begin{array}{l}* \\
3.4 \\
5.7\end{array}$ & $\begin{array}{r}12.2 \\
4.4 \\
4.5\end{array}$ & $\begin{array}{r}8.3 \\
24.3 \\
17.8\end{array}$ & $\begin{array}{c}* \\
13.9 \\
7.5\end{array}$ & $\begin{array}{l}* \\
.8 \\
*\end{array}$ & $\begin{array}{l}* \\
7.2 \\
5.4\end{array}$ & $\begin{array}{l}* \\
4.9 \\
5.7\end{array}$ \\
\hline $\begin{array}{l}\text { Years under current } \\
\text { ownership } \\
0-4 \ldots \ldots \ldots \ldots \ldots \\
5-9 \ldots \ldots \ldots \ldots \ldots \ldots \\
10-14 \ldots \ldots \ldots \ldots \ldots \ldots \\
15-19 \ldots \ldots \ldots \ldots \ldots \ldots \\
20-24 \ldots \ldots \ldots \ldots \ldots \\
25 \text { or more } \ldots \ldots \ldots \ldots\end{array}$ & $\begin{array}{l}96.1 \\
95.8 \\
98.1 \\
94.7 \\
96.0 \\
97.8\end{array}$ & $\begin{array}{l}95.0 \\
95.4 \\
97.7 \\
94.1 \\
95.3 \\
97.3\end{array}$ & $\begin{array}{l}83.5 \\
86.5 \\
88.5 \\
83.6 \\
85.1 \\
90.9\end{array}$ & $\begin{array}{l}14.2 \\
12.2 \\
12.4 \\
15.7 \\
15.7 \\
14.0\end{array}$ & $\begin{array}{r}9.3 \\
7.7 \\
10.5 \\
5.3 \\
6.6 \\
8.0\end{array}$ & $\begin{array}{l}51.1 \\
56.2 \\
58.4 \\
54.7 \\
55.4 \\
49.7\end{array}$ & $\begin{array}{l}31.8 \\
39.6 \\
45.8 \\
44.8 \\
45.1 \\
40.0\end{array}$ & $\begin{array}{l}23.3 \\
25.9 \\
26.5 \\
27.1 \\
25.3 \\
23.8\end{array}$ & $\begin{array}{r}5.5 \\
12.4 \\
20.9 \\
18.8 \\
18.8 \\
18.5\end{array}$ & $\begin{array}{l}4.3 \\
4.6 \\
5.9 \\
3.4 \\
4.8 \\
3.8\end{array}$ & $\begin{array}{l}3.3 \\
4.7 \\
4.4 \\
6.5 \\
8.2 \\
7.1\end{array}$ & $\begin{array}{l}29.1 \\
27.7 \\
22.6 \\
23.3 \\
21.4 \\
18.5\end{array}$ & $\begin{array}{r}15.7 \\
15.0 \\
12.6 \\
15.4 \\
12.5 \\
8.6\end{array}$ & $\begin{array}{c}* \\
* \\
* \\
* \\
* \\
1.2\end{array}$ & $\begin{array}{l}9.5 \\
8.0 \\
4.3 \\
5.3 \\
5.0 \\
5.5\end{array}$ & $\begin{array}{l}5.3 \\
6.8 \\
5.9 \\
4.9 \\
4.6 \\
4.6\end{array}$ \\
\hline
\end{tabular}

1998, 62 percent of small businesses reported using trade credit. Use generally increased with firm size, rising from about one-third of the smallest firms to more than 85 percent of the largest firms. Young firms, proprietorships, and firms owned by nonwhites, Hispanics, or females were less likely than others to use this service. The differences between these groups of firms and other groups are similar to the differences in use between smaller and larger firms.

The use of trade credit was most common among firms in construction, manufacturing, and wholesale and retail trade-industries for which nonlabor expenses, such those for equipment and inventory, are large relative to labor costs. Among industries for 
9.-Continued

Percent

\begin{tabular}{|c|c|c|c|c|c|c|c|c|c|c|c|c|c|c|c|c|}
\hline \multirow[b]{3}{*}{ Characteristic } & \multirow[b]{3}{*}{$\begin{array}{c}\text { Any } \\
\text { supplier }\end{array}$} & \multirow{2}{*}{\multicolumn{4}{|c|}{ Depository }} & \multicolumn{11}{|c|}{ Nondepository } \\
\hline & & & & & & \multirow[b]{2}{*}{ Any } & \multicolumn{5}{|c|}{ Primarily financial } & \multicolumn{5}{|c|}{ Other nondepository } \\
\hline & & Any & $\begin{array}{c}\text { Com- } \\
\text { mercial } \\
\text { bank }\end{array}$ & $\begin{array}{c}\text { Thrift } \\
\text { institu- } \\
\text { tion }^{1}\end{array}$ & $\begin{array}{l}\text { Credit } \\
\text { union }\end{array}$ & & Any & $\begin{array}{c}\text { Finance } \\
\text { com- } \\
\text { pany or } \\
\text { factor }\end{array}$ & $\begin{array}{l}\text { Broker- } \\
\text { age or } \\
\text { pension } \\
\text { firm }\end{array}$ & $\begin{array}{l}\text { Leasing } \\
\text { com- } \\
\text { pany }\end{array}$ & $\begin{array}{l}\text { Insur- } \\
\text { ance or } \\
\text { mort- } \\
\text { gage } \\
\text { com- } \\
\text { pany }\end{array}$ & Any & $\begin{array}{l}\text { Card or } \\
\text { check } \\
\text { pro- } \\
\text { cessing } \\
\text { firm }^{2}\end{array}$ & $\begin{array}{c}\text { Govern- } \\
\text { ment }\end{array}$ & \begin{tabular}{|l} 
Family \\
and in- \\
divid- \\
uals
\end{tabular} & Other $^{3}$ \\
\hline $\begin{array}{l}\text { Census area } \\
\text { of main office } \\
\text { Northeast ............. } \\
\quad \text { New England ..... } \\
\text { Middle Atlantic ... }\end{array}$ & $\begin{array}{l}95.5 \\
97.9 \\
94.4\end{array}$ & $\begin{array}{l}94.6 \\
97.5 \\
93.4\end{array}$ & $\begin{array}{l}77.4 \\
63.9 \\
83.2\end{array}$ & $\begin{array}{l}28.4 \\
51.7 \\
18.3\end{array}$ & $\begin{array}{l}6.3 \\
* \\
6.2\end{array}$ & $\begin{array}{l}54.9 \\
53.2 \\
55.6\end{array}$ & $\begin{array}{l}40.2 \\
40.7 \\
40.0\end{array}$ & $\begin{array}{l}26.1 \\
28.8 \\
25.0\end{array}$ & $\begin{array}{l}17.6 \\
16.6 \\
18.1\end{array}$ & $\begin{array}{l}3.1 \\
4.0 \\
2.8\end{array}$ & $\begin{array}{l}4.6 \\
6.1 \\
3.9\end{array}$ & $\begin{array}{l}25.2 \\
23.9 \\
25.8\end{array}$ & $\begin{array}{l}14.8 \\
16.5 \\
14.1\end{array}$ & $\begin{array}{l}* \\
* \\
*\end{array}$ & $\begin{array}{l}6.9 \\
* \\
7.1\end{array}$ & $\begin{array}{c}4.7 \\
* \\
5.7\end{array}$ \\
\hline $\begin{array}{l}\text { Midwest ............... } \\
\text { East North Central } \\
\text { West North Central }\end{array}$ & $\begin{array}{l}98.4 \\
98.0 \\
99.3\end{array}$ & $\begin{array}{l}98.1 \\
97.6 \\
99.3\end{array}$ & $\begin{array}{l}92.4 \\
91.2 \\
94.7\end{array}$ & $\begin{array}{l}7.8 \\
9.6 \\
4.0\end{array}$ & $\begin{array}{l}9.5 \\
9.8 \\
8.9\end{array}$ & $\begin{array}{l}55.2 \\
55.8 \\
54.0\end{array}$ & $\begin{array}{l}41.9 \\
43.1 \\
39.3\end{array}$ & $\begin{array}{l}22.9 \\
21.5 \\
25.8\end{array}$ & $\begin{array}{l}17.8 \\
19.0 \\
15.4\end{array}$ & $\begin{array}{l}5.2 \\
5.9 \\
3.7\end{array}$ & $\begin{array}{l}5.8 \\
5.4 \\
6.7\end{array}$ & $\begin{array}{l}24.1 \\
24.8 \\
22.6\end{array}$ & $\begin{array}{l}13.0 \\
12.2 \\
14.8\end{array}$ & $\begin{array}{c}2.6 \\
* \\
*\end{array}$ & $\begin{array}{l}4.7 \\
5.3 \\
3.5\end{array}$ & $\begin{array}{l}6.2 \\
6.3 \\
6.0\end{array}$ \\
\hline $\begin{array}{r}\text { South ..................... } \\
\text { South Atlantic .... } \\
\text { East South Central } \\
\text { West South Central }\end{array}$ & $\begin{array}{l}96.5 \\
97.5 \\
94.9 \\
95.5\end{array}$ & $\begin{array}{l}95.8 \\
96.7 \\
94.9 \\
94.7\end{array}$ & $\begin{array}{l}89.0 \\
88.8 \\
89.5 \\
89.1\end{array}$ & $\begin{array}{c}9.8 \\
12.3 \\
* \\
7.7\end{array}$ & $\begin{array}{l}7.0 \\
5.6 \\
* \\
7.1\end{array}$ & $\begin{array}{l}55.7 \\
60.0 \\
45.7 \\
52.9\end{array}$ & $\begin{array}{l}40.8 \\
44.9 \\
33.2 \\
37.1\end{array}$ & $\begin{array}{l}27.4 \\
30.1 \\
21.6 \\
25.7\end{array}$ & $\begin{array}{l}11.6 \\
12.5 \\
11.0 \\
10.2\end{array}$ & $\begin{array}{l}5.3 \\
6.0 \\
4.3 \\
4.6\end{array}$ & $\begin{array}{l}5.9 \\
6.4 \\
4.7 \\
5.5\end{array}$ & $\begin{array}{l}24.8 \\
26.6 \\
19.6 \\
24.0\end{array}$ & $\begin{array}{r}13.9 \\
15.9 \\
9.5 \\
12.4\end{array}$ & $\begin{array}{l}.7 \\
* \\
* \\
*\end{array}$ & $\begin{array}{l}7.0 \\
6.3 \\
* \\
8.3\end{array}$ & $\begin{array}{l}5.3 \\
5.7 \\
4.4 \\
5.1\end{array}$ \\
\hline $\begin{array}{l}\text { West } \ldots \ldots \ldots \ldots \ldots \\
\quad \text { Mountain } \ldots \ldots \ldots \ldots \\
\text { Pacific } \ldots \ldots \ldots \ldots \ldots\end{array}$ & $\begin{array}{l}95.4 \\
95.6 \\
95.4\end{array}$ & $\begin{array}{l}94.9 \\
94.8 \\
95.0\end{array}$ & $\begin{array}{l}85.2 \\
90.0 \\
83.0\end{array}$ & $\begin{array}{r}12.8 \\
7.1 \\
15.4\end{array}$ & $\begin{array}{r}10.0 \\
9.5 \\
10.2\end{array}$ & $\begin{array}{l}50.1 \\
52.1 \\
49.2\end{array}$ & $\begin{array}{l}38.3 \\
37.9 \\
38.5\end{array}$ & $\begin{array}{l}23.1 \\
26.5 \\
21.5\end{array}$ & $\begin{array}{l}14.9 \\
10.3 \\
17.0\end{array}$ & $\begin{array}{l}3.7 \\
2.1 \\
4.5\end{array}$ & $\begin{array}{l}4.9 \\
4.6 \\
5.1\end{array}$ & $\begin{array}{l}23.0 \\
24.7 \\
22.3\end{array}$ & $\begin{array}{l}11.9 \\
11.3 \\
12.2\end{array}$ & $\begin{array}{c}1.3 \\
* \\
.6\end{array}$ & $\begin{array}{l}7.5 \\
9.8 \\
6.5\end{array}$ & $\begin{array}{l}5.7 \\
5.8 \\
5.7\end{array}$ \\
\hline $\begin{array}{l}\text { Urbanization } \\
\text { at main office } \\
\text { Urban } \ldots \ldots \ldots \ldots \ldots \\
\text { Rural ......................... }\end{array}$ & $\begin{array}{l}96.5 \\
96.2\end{array}$ & $\begin{array}{l}95.9 \\
95.9\end{array}$ & $\begin{array}{l}86.2 \\
87.5\end{array}$ & $\begin{array}{l}14.2 \\
12.2\end{array}$ & $\begin{array}{l}7.7 \\
9.9\end{array}$ & $\begin{array}{l}55.4 \\
48.7\end{array}$ & $\begin{array}{l}41.9 \\
34.3\end{array}$ & $\begin{array}{l}25.7 \\
23.0\end{array}$ & $\begin{array}{l}15.8 \\
11.7\end{array}$ & $\begin{array}{l}4.8 \\
3.3\end{array}$ & $\begin{array}{l}5.5 \\
4.8\end{array}$ & $\begin{array}{l}25.1 \\
21.3\end{array}$ & $\begin{array}{l}13.7 \\
12.5\end{array}$ & $\begin{array}{r}1.3 \\
.8\end{array}$ & $\begin{array}{l}6.7 \\
6.4\end{array}$ & $\begin{array}{l}6.0 \\
3.3\end{array}$ \\
\hline $\begin{array}{l}\text { Number of offices } \\
\text { One } \ldots \ldots \ldots \ldots \ldots \\
\text { Two } \ldots \ldots \ldots \ldots \ldots \ldots \\
\text { Three or more ....... }\end{array}$ & $\begin{array}{r}95.9 \\
99.2 \\
100.0\end{array}$ & $\begin{array}{l}95.4 \\
98.7 \\
99.2\end{array}$ & $\begin{array}{l}85.5 \\
90.8 \\
96.3\end{array}$ & $\begin{array}{r}14.0 \\
14.3 \\
9.0\end{array}$ & $\begin{array}{r}7.8 \\
10.0 \\
11.0\end{array}$ & $\begin{array}{l}52.0 \\
68.9 \\
62.7\end{array}$ & $\begin{array}{l}38.4 \\
50.6 \\
54.0\end{array}$ & $\begin{array}{l}24.6 \\
28.7 \\
28.8\end{array}$ & $\begin{array}{l}13.7 \\
19.0 \\
29.0\end{array}$ & $\begin{array}{r}3.6 \\
9.2 \\
10.5\end{array}$ & $\begin{array}{r}5.2 \\
4.8 \\
10.0\end{array}$ & $\begin{array}{l}23.1 \\
34.6 \\
25.5\end{array}$ & $\begin{array}{r}13.3 \\
16.7 \\
9.3\end{array}$ & $\begin{array}{c}.8 \\
* \\
2.4\end{array}$ & $\begin{array}{r}6.0 \\
11.5 \\
7.4\end{array}$ & $\begin{array}{l}5.1 \\
7.0 \\
8.8\end{array}$ \\
\hline $\begin{array}{l}\text { Sales area } \\
\text { Primarily within the } \\
\text { United States .. } \\
\text { International or } \\
\text { olobal }\end{array}$ & 96.4 & 95.9 & 86.7 & 13.8 & 8.1 & 53.8 & 40.1 & 25.0 & 14.7 & 4.4 & 5.3 & 24.0 & 13.2 & 1.2 & 6.5 & 5.5 \\
\hline $\begin{array}{l}\text { Management } \\
\text { By owner ........... } \\
\text { Hired ............ }\end{array}$ & $\begin{array}{l}96.2 \\
99.9\end{array}$ & $\begin{array}{l}95.6 \\
99.9\end{array}$ & $\begin{array}{l}86.0 \\
93.4\end{array}$ & $\begin{array}{l}13.6 \\
16.7\end{array}$ & $\begin{array}{l}8.2 \\
7.6\end{array}$ & $\begin{array}{l}53.0 \\
69.3\end{array}$ & $\begin{array}{l}39.3 \\
56.2\end{array}$ & $\begin{array}{l}24.6 \\
34.5\end{array}$ & $\begin{array}{l}14.3 \\
24.7\end{array}$ & $\begin{array}{l}4.4 \\
5.3\end{array}$ & $\begin{array}{r}5.0 \\
10.5\end{array}$ & $\begin{array}{l}24.0 \\
28.9\end{array}$ & $\begin{array}{l}13.2 \\
16.9\end{array}$ & $\begin{array}{c}1.2 \\
*\end{array}$ & $\begin{array}{l}6.7 \\
5.3\end{array}$ & $\begin{array}{l}5.2 \\
8.9\end{array}$ \\
\hline $\begin{array}{l}\text { Race, ethnicity, and } \\
\text { sex of majority owners } \\
\text { Nonwhite or Hispanic .. } \\
\text { Non-Hispanic white ... }\end{array}$ & $\begin{array}{l}95.5 \\
96.5\end{array}$ & $\begin{array}{l}94.9 \\
95.9\end{array}$ & $\begin{array}{l}85.8 \\
86.5\end{array}$ & $\begin{array}{l}12.0 \\
14.0\end{array}$ & $\begin{array}{l}7.4 \\
8.2\end{array}$ & $\begin{array}{l}53.3 \\
53.7\end{array}$ & $\begin{array}{l}35.3 \\
40.6\end{array}$ & $\begin{array}{l}25.7 \\
24.7\end{array}$ & $\begin{array}{l}10.9 \\
15.3\end{array}$ & $\begin{array}{l}2.1 \\
4.8\end{array}$ & $\begin{array}{l}4.9 \\
5.3\end{array}$ & $\begin{array}{l}27.5 \\
23.6\end{array}$ & $\begin{array}{l}15.0 \\
13.1\end{array}$ & $\stackrel{*}{1.2}$ & $\begin{array}{l}7.8 \\
6.4\end{array}$ & $\begin{array}{l}5.1 \\
5.5\end{array}$ \\
\hline $\begin{array}{l}\text { White } \ldots \ldots \ldots \ldots \ldots \\
\text { Black ........................ } \\
\text { Asian, Native Hawaiian, } \\
\quad \text { or other Pacific }\end{array}$ & $\begin{array}{l}96.5 \\
91.7\end{array}$ & $\begin{array}{l}95.9 \\
91.7\end{array}$ & $\begin{array}{l}86.3 \\
81.0\end{array}$ & $\begin{array}{l}13.9 \\
13.7\end{array}$ & $\begin{array}{l}8.4 \\
*\end{array}$ & $\begin{array}{l}53.9 \\
46.2\end{array}$ & $\begin{array}{l}40.6 \\
36.0\end{array}$ & $\begin{array}{l}25.0 \\
28.9\end{array}$ & $\begin{array}{c}15.3 \\
*\end{array}$ & $\begin{array}{l}4.6 \\
*\end{array}$ & $\begin{array}{l}5.4 \\
*\end{array}$ & $\begin{array}{l}23.7 \\
20.6\end{array}$ & $\begin{array}{c}13.2 \\
*\end{array}$ & $\begin{array}{c}1.2 \\
*\end{array}$ & $\begin{array}{l}6.4 \\
*\end{array}$ & $\begin{array}{l}5.5 \\
*\end{array}$ \\
\hline $\begin{array}{c}\text { Islander ............. } \\
\text { American Indian or } \\
\text { Alaska Native } \ldots . .\end{array}$ & 99.1 & 97.3 & 93.6 & $\begin{array}{c}13.0 \\
*\end{array}$ & * & 59.8 & 33.9 & 22.9 & 12.3 & * & * & 36.2 & $\begin{array}{c}23.8 \\
*\end{array}$ & * & * & $\begin{array}{l}* \\
*\end{array}$ \\
\hline $\begin{array}{l}\text { Hispanic } \ldots \ldots \ldots \ldots \ldots \\
\text { Non-Hispanic } \ldots \ldots \ldots \ldots\end{array}$ & $\begin{array}{l}94.9 \\
96.5\end{array}$ & $\begin{array}{l}94.9 \\
95.9\end{array}$ & $\begin{array}{l}82.4 \\
86.6\end{array}$ & $\begin{array}{c}* \\
13.9\end{array}$ & $\begin{array}{l}* \\
8.0\end{array}$ & $\begin{array}{l}54.7 \\
53.9\end{array}$ & $\begin{array}{l}40.0 \\
40.3\end{array}$ & $\begin{array}{l}28.9 \\
25.0\end{array}$ & $\begin{array}{l}15.1 \\
14.9\end{array}$ & $\begin{array}{l}* \\
4.6\end{array}$ & $\begin{array}{l}7.6 \\
5.2\end{array}$ & $\begin{array}{l}25.4 \\
24.2\end{array}$ & $\begin{array}{l}14.1 \\
13.4\end{array}$ & $\begin{array}{c}* \\
1.3\end{array}$ & $\begin{array}{l}* \\
6.6\end{array}$ & $\stackrel{*}{5.5}$ \\
\hline $\begin{array}{l}\text { Female } \ldots \ldots \ldots \ldots \ldots \\
\text { Male } \ldots \ldots \ldots \ldots \ldots \ldots \\
\text { Ownership divided }\end{array}$ & $\begin{array}{l}92.3 \\
97.4\end{array}$ & $\begin{array}{l}91.4 \\
96.8\end{array}$ & $\begin{array}{l}79.8 \\
88.2\end{array}$ & $\begin{array}{l}13.9 \\
13.2\end{array}$ & $\begin{array}{l}8.2 \\
8.1\end{array}$ & $\begin{array}{l}45.0 \\
57.1\end{array}$ & $\begin{array}{l}30.5 \\
44.0\end{array}$ & $\begin{array}{l}20.3 \\
26.8\end{array}$ & $\begin{array}{l}10.5 \\
17.0\end{array}$ & $\begin{array}{l}3.4 \\
4.8\end{array}$ & $\begin{array}{l}2.7 \\
6.1\end{array}$ & $\begin{array}{l}23.9 \\
24.4\end{array}$ & $\begin{array}{l}14.2 \\
12.9\end{array}$ & $\begin{array}{l}1.1 \\
1.2\end{array}$ & $\begin{array}{l}6.5 \\
6.9\end{array}$ & $\begin{array}{l}3.7 \\
6.1\end{array}$ \\
\hline equally by sex .... & 98.6 & 98.6 & 89.1 & 16.8 & 8.2 & 53.8 & 38.6 & 25.1 & 12.1 & 4.6 & 6.4 & 24.3 & 14.4 & * & 5.3 & 5.1 \\
\hline
\end{tabular}

Note: For definition of sales areas, refer to table 2, note 3.

1. Savings bank or savings association.

2. Includes credit and debit card processing firms.

3. Includes business firms, suppliers, and venture capital firms.

* Fewer than fifteen observations.

which labor's share of costs is high, such as business and professional services, the use of trade credit was somewhat less common.

As indicated earlier, trade credit can be used for transaction purposes and for financing. Some of the use patterns (for example, variation by industry) are more consistent with the transaction hypothesis. Among the firms that reported using trade credit in 2003, 59 percent reported that they always paid the debt by the due date. The firms that did not always pay on time paid late only about 30 percent of the time, which suggests that, even for these firms, the major use of trade credit was for transaction purposes.

\section{SUPPLIERS OF FINANCIAL SERVICES USED BY SMALL BUSINESSES}

The suppliers of financial services to small businesses consist of depository institutions — commercial banks, 
thrift institutions (savings banks and savings associations), and credit unions-and nondepository institutions. Nondepositories consist of primarily financial nondepositories - finance companies and factors, brokerage and pension firms, leasing companies, and insurance and mortgage companies-and other nondepositories - credit card and check processing firms, government sources, family and individuals, business firms, suppliers, venture capital firms, and miscellaneous types. The survey collected information on the sources of checking and savings accounts; lines of credit, loans, and capital leases; and financial management services. ${ }^{28}$

In 2003, depository institutions were used by 96 percent of all firms, roughly the same percentage as in 1998 (table 9). Among depository institutions, the use of commercial banks remained about the same, whereas the use of thrift institutions and credit unions increased.

In contrast, the proportion of firms using nondepository institutions increased from 40 percent in 1998 to 54 percent in 2003, with increases recorded both for primarily financial nondepository sources and for other nondepository sources. Among the primarily financial nondepository sources, only leasing companies were used somewhat less in 2003 than in 1998. Among other nondepository institutions, credit card processors logged the largest increase between 1998 and 2003. ${ }^{29}$ These changes are consistent with the finding that the percentage of small businesses that used credit card processing services increased over the period between surveys.

\section{Depository Financial Institutions}

Depository institutions provided at least one financial service to about 96 percent of small businesses in 2003 (table 9), roughly the same percentage of businesses that had a checking or savings account in 2003 (table 6.A). Commercial banks continued to be used by a far larger proportion of firms ( 87 percent) than were thrift institutions (14 percent) or credit unions

28. No information on the sources of owner loans, credit cards, and trade credit was collected.

29. As noted earlier, an additional service-credit card and debit card processing-was added to the list of financial management services in the 2003 survey. This additional service may have been partly responsible for the observed increase in the use of nondepository sources-especially of credit card processing sources. However, the data suggest that part of the increase in the use of nondepository sources was independent of the addition of the credit card processing service. For most types of nondepository sources, including finance companies, brokerages, insurance and mortgage companies, government, and family and individuals, the incidence increased between 1998 and 2003. The only nondepository sources for which incidence decreased were leasing companies and "other" types.
(8 percent). In general, the percentage of firms using commercial banks increased with firm size, and once a certain size threshold was crossed (for example, at least twenty employees or at least $\$ 2.5$ million in sales), virtually all firms (97 percent or more) used at least one commercial bank. In contrast, the use of credit unions declined with size, and the use of thrift institutions did not vary systematically with size. Proprietorships, which are generally the smallest firms, were somewhat less likely than other firm types to use commercial banks and more likely to use credit unions and thrift institutions.

The use of thrift institutions increased from 9 percent to nearly 14 percent between 1998 and 2003, and the use of credit unions increased from 6 percent to 8 percent. The increased use of thrift institutions reverses declines observed in earlier surveys, declines that had been attributed to the decrease in the number of thrifts during the 1990s. The increased use of thrifts and credit unions suggests that the deregulation of business lending by those institutions and the expansion in potential credit union membership permitted by the relaxation in the definition of "common bonds" by the National Credit Union Administration in recent years may have enabled these institutions to better meet the financial service demands of small businesses.

As was true in earlier surveys, small businesses in New England were much more likely to use thrift institutions (52 percent) than were firms in other parts of the country. This finding is consistent with the fact that thrifts account for a larger proportion of depositories in New England than in other areas of the country. Moreover, thrifts in New England tend to look more like commercial banks in terms of their business lending than do thrifts outside of New England. ${ }^{30}$ Credit unions were most likely to have been used by firms located in the Pacific part of the West.

Black-owned and Hispanic-owned businesses were less likely than non-Hispanic-owned or white-owned firms to use commercial banks. Asian-owned firms were more likely to use commercial banks than other ownership groups. Also, female-owned firms were less likely than male-owned firms to use commercial banks. In 1998, the groups most likely to use commercial banks were also the least likely to use thrift institutions and credit unions. In 2003, this apparent substitution across institution types altered somewhat. The use of thrifts and credit unions was often greatest for those firm types that were also the most

30. Steven J. Pilloff and Robin A. Prager (1998), “Thrift Involvement in Commercial and Industrial Lending," Federal Reserve Bulletin, vol. 84 (December), pp. 1025-37. 
likely to use commercial banks. For example, the use of thrifts was highest for white-owned firms and for firms owned equally by males and females; the use of credit unions was highest for white-owned firms and for male-owned firms.

\section{Nondepository Sources}

Small businesses obtained their financial services from a variety of sources besides depository institutions. Between 1998 and 2003, the incidence of nondepository use by small firms increased from 40 percent to 54 percent (table 9). Both primarily financial nondepositories and other nondepositories were used more frequently in 2003 than in 1998.

\section{Primarily Financial Nondepositories}

Primarily financial nondepositories were a source of financial services for about 40 percent of all small firms, a sizable increase over the roughly 30 percent incidence reported in 1998. And as in 1998, the most commonly used financial nondepositories were finance companies, followed by brokerage companies.

The use of financial nondepositories (and of each subgroup of suppliers within that group) is strongly related to firm size, increasing from about one-fourth of the smallest firms to about three-fourths of the largest firms. For example, 16 percent of the smallest firms (those with fewer than 2 employees) used finance companies, and 8 percent used brokerages, whereas about 42 percent of firms with 100 or more employees used finance companies and 41 percent used brokerage companies. Use of financial nondepositories also differed by organizational form, ranging from 31 percent of proprietorships to 52 percent of $\mathrm{C}$ corporations. Proprietorships and partnerships were about half as likely as corporations to use brokerages and about two-thirds as likely to use finance companies.

The use of financial nondepositories also varied with the race, ethnicity, and sex of the business owners. White-owned and male-owned firms used financial nondepositories, finance companies, brokerages, and leasing companies more often than did other types of firms. The differences were largest among groups using brokerage firms: For example, 15 percent of white-owned firms used brokerages, compared with 11 percent of nonwhite or Hispanicowned firms.

\section{Other Nondepositories}

In 2003, 24 percent of firms used other nondepositories (such as card and check processors, government, family and individuals, other businesses, supplier businesses, and venture capital firms), up from 16 percent in 1998. Most of this increase is due to a rise in the use of card and check processors (from 4 percent in 1998 to 13 percent in 2003), which may, in turn, partly reflect increased use and acceptance of credit and debit cards by small businesses. ${ }^{31}$ The use of other subgroups was largely unchanged from 1998. About 1 percent used government, 7 percent used family and individuals, and 6 percent used other sources, including other business firms. ${ }^{32}$

The use of other nondepository sources did not consistently increase with firm size, although it was least likely for the smallest firms. For example, the use of other nondepository sources increased from 15 percent of firms with fewer than two employees to 33 percent for firms with five to nine employees. For larger firms, the percentage using such sources generally remained at the higher levels. Proprietorships and partnerships were less likely than were corporations to use these sources.

Younger firms were more likely to use other nondepositories, including family and individuals, than were older firms. Younger firms sometimes have difficulty borrowing from financial institutions, in part because financial institutions often require that prospective borrowers provide several years of financial statements with their loan applications. Nonfinancial sources, such as individuals who may be familiar with the prospective borrowers, may be better positioned to evaluate creditworthiness and to monitor the financial condition of younger firms, or alternatively they may have different credit standards than financial sources. The survey results show that in 2003 the use of family and individuals was most common among younger firms.

\section{USE OF FINANCIAL SERVICES SUPPLIERS, BY SERVICE}

The data reviewed thus far have examined variations in the use of financial services by firms according to characteristics of the firm and variations in the source of financial services used by firms according to the type of firm. This section reports on the types of

31. As indicated earlier, some of the observed increase is likely caused by explicitly asking about credit card processing services in the 2003 survey. Before 2003, credit card processing was included in the question about transaction services.

32. The 1 percent figure likely understates the true role of government in providing financial services to small businesses. Many government entities, such as the U.S. Small Business Administration, provide credit guarantees, which ensure repayment of small business loans made by institutional lenders such as commercial banks and thrift institutions. 
10. Use of selected suppliers of financial services by small businesses, by selected service, 2003 survey Percent

\begin{tabular}{|c|c|c|c|c|c|c|c|c|c|c|c|c|c|c|c|c|}
\hline \multirow[b]{3}{*}{ Service } & \multirow[b]{3}{*}{$\begin{array}{c}\text { Any } \\
\text { supplier }\end{array}$} & \multirow{2}{*}{\multicolumn{4}{|c|}{ Depository }} & \multicolumn{11}{|c|}{ Nondepository } \\
\hline & & & & & & \multirow[b]{2}{*}{ Any } & \multicolumn{5}{|c|}{ Primarily financial } & \multicolumn{5}{|c|}{ Other nondepository } \\
\hline & & Any & $\begin{array}{c}\text { Com- } \\
\text { mercial } \\
\text { bank }\end{array}$ & $\begin{array}{l}\text { Thrift } \\
\text { institu- } \\
\text { tion }\end{array}$ & $\begin{array}{l}\text { Credit } \\
\text { union }\end{array}$ & & Any & $\begin{array}{c}\text { Finance } \\
\text { com- } \\
\text { pany or } \\
\text { factor }\end{array}$ & $\begin{array}{c}\text { Broker- } \\
\text { age or } \\
\text { pension } \\
\text { firm }\end{array}$ & $\begin{array}{l}\text { Leasing } \\
\text { com- } \\
\text { pany }\end{array}$ & $\begin{array}{c}\text { Insur- } \\
\text { ance or } \\
\text { mort- } \\
\text { gage } \\
\text { com- } \\
\text { pany }\end{array}$ & Any & $\begin{array}{c}\text { Card or } \\
\text { check } \\
\text { pro- } \\
\text { cessing } \\
\text { firm }\end{array}$ & $\begin{array}{c}\text { Govern- } \\
\text { ment }\end{array}$ & $\begin{array}{c}\text { Family } \\
\text { and in- } \\
\text { divid- } \\
\text { uals }\end{array}$ & Other \\
\hline Any $\ldots \ldots \ldots \ldots \ldots \ldots$ & 96.4 & 95.9 & 86.5 & 13.8 & 8.1 & 54.1 & 40.3 & 25.2 & 14.9 & 4.5 & 5.4 & 24.3 & 13.4 & 1.2 & 6.6 & 5.5 \\
\hline Liquid asset account & 95.0 & 94.5 & 83.2 & 10.9 & 4.9 & 4.5 & 4.2 & .4 & 3.8 & 0 & .1 & .3 & .1 & 0 & 0 & .2 \\
\hline Checking...$\ldots \ldots$ & 94.6 & 94.1 & 82.7 & 10.5 & 4.4 & 1.8 & 1.7 & .1 & 1.6 & 0 & 0 & .2 & .1 & 0 & 0 & .1 \\
\hline Savings . & 22.1 & 20.0 & 16.2 & 2.4 & 2.3 & 3.1 & 3.0 & .4 & 2.6 & 0 & .1 & .1 & .0 & 0 & 0 & .1 \\
\hline $\begin{array}{l}\text { Credit line, loan, } \\
\text { or capital lease }\end{array}$ & 60.4 & 46.4 & 41.1 & 5.5 & 3.9 & 33.3 & 26.6 & 22.2 & .8 & 4.3 & 2.3 & 10.4 & .3 & 1.1 & 6.5 & 2.9 \\
\hline Credit line...... & 34.3 & 32.4 & 29.5 & 2.8 & .9 & 3.6 & 2.9 & 2.2 & .4 & .1 & .4 & 8 & 0 & .1 & .1 & .6 \\
\hline Mortgage...... & 13.3 & 10.8 & 9.1 & 1.9 & .3 & 3.4 & 1.9 & .6 & .1 & 0 & 1.2 & 1.5 & 0 & .4 & .9 & .2 \\
\hline Vehicle loan .... & 25.5 & 13.2 & 10.0 & 1.1 & 2.5 & 15.2 & 14.8 & 14.5 & 0 & .3 & .1 & .4 & 0 & 0 & .2 & .2 \\
\hline Equipment loan . & 10.3 & 5.1 & 4.3 & .5 & .4 & 5.8 & 4.4 & 3.4 & .2 & 1.0 & .1 & 1.5 & .1 & .1 & .5 & .8 \\
\hline Capital lease .... & 8.7 & 1.1 & 1.0 & .1 & 0 & 7.8 & 6.9 & 4.6 & 0 & 2.9 & 0 & 1.1 & .1 & 0 & .1 & 1.0 \\
\hline Other $\ldots \ldots \ldots$ & 10.1 & 3.4 & 3.1 & .2 & .2 & 7.2 & 1.4 & .6 & .1 & .2 & .6 & 5.9 & 0 & .5 & 4.9 & .5 \\
\hline Financial management & 64.8 & 53.2 & 48.2 & 5.6 & 2.2 & 33.1 & 20.1 & 5.1 & 13.3 & .3 & 3.2 & 16.3 & 13.3 & .1 & .3 & 2.8 \\
\hline Transaction $\ldots \ldots \ldots$ & 39.0 & 38.3 & 34.1 & 4.0 & 1.9 & 3.0 & 2.0 & .8 & 1.0 & 0 & .2 & 1.1 & .6 & 0 & .2 & .4 \\
\hline processing ...... & 37.2 & 22.5 & 20.7 & 1.7 & .4 & 16.7 & 3.1 & 2.8 & .1 & .1 & .2 & 14.2 & 13.1 & 0 & 0 & 1.0 \\
\hline Cash management ... & 6.7 & 6.2 & 5.8 & .4 & .1 & .8 & .7 & .2 & .5 & 0 & 0 & .1 & 0 & .1 & 0 & 0 \\
\hline Credit-related $\ldots$. & 5.0 & 4.1 & 3.8 & .4 & .2 & 1.4 & 1.0 & .8 & .1 & .1 & 0 & .4 & .1 & 0 & 0 & .3 \\
\hline Brokerage ........ & 5.6 & .8 & .8 & 0 & .1 & 5.0 & 4.9 & .3 & 4.5 & 0 & .3 & .2 & 0 & 0 & 0 & .2 \\
\hline Trust and pension & 17.2 & 3.6 & 3.2 & .4 & .2 & 14.3 & 13.2 & .8 & 10.1 & .1 & 2.7 & 1.2 & .1 & 0 & .1 & 1.0 \\
\hline
\end{tabular}

NoTE: Refer to numbered notes to table 9.

financial services provided to small businesses by each type of financial service supplier.

Not all financial service suppliers provide the full range of financial services (table 10). Depository institutions were used for the full range of serviceschecking and savings (95 percent of firms); lines, loans, and leases (46 percent); and financial management services (53 percent). In fact, a much larger percentage of firms used depository institutions for each of these services than used nondepository sources. One in four firms obtained lines, loans, or leases from primarily financial nondepositories, and one in five firms obtained financial management services from primarily financial nondepositories. Among other nondepositories, used by about one in four firms, credit card processors were important sources of financial management services, and family and individuals were most often used for credit.

\section{Checking and Savings Accounts}

As was the case in previous surveys, commercial banks dominated the provision of checking accounts to small businesses in 2003, supplying them to 83 percent of all small firms (table 10). Firms also obtained checking accounts from thrift institutions (11 percent), credit unions ( 4 percent), and brokerages ( 2 percent). No other type of supplier provided more than a trivial share of checking accounts. Commercial banks were also the dominant supplier of savings accounts, far outpacing the next most common providers (thrifts, credit unions, and brokerages).

\section{Credit Lines, Loans, and Capital Leases}

In 2003, commercial banks were the most common supplier of credit lines, loans, and capital leases, providing about 41 percent of firms with such services, slightly up from 39 percent in 1998 (table 10). Primarily financial nondepositories and family and individuals were also important suppliers. In 2003, one-fourth of businesses obtained lines from, or had outstanding balances on loans or leases with, primarily financial nondepositories (specifically, finance and leasing companies); 7 percent had loans from family and individuals. These percentages were somewhat lower in 1998. Although suppliers other than commercial banks were important sources of lines, loans, and leases, commercial banks in 2003 were about two times more likely than finance companies to have been the source of these services for small businesses, six times more likely than family and individuals, and ten times more likely than leasing companies.

Credit lines, used by about one-third of businesses, were the most commonly used form of credit. They were supplied primarily by commercial banks, thrift institutions, and finance companies. Commercial 
banks were the most important source and were ten times more likely than thrifts and fifteen times more likely than finance companies to have been the source of this service. Most mortgages used for business purposes were obtained from commercial banks ( 9 percent of firms), thrifts ( 2 percent), mortgage companies ( 1 percent), and family and individuals ( 1 percent). Vehicle loans were obtained primarily from finance companies (15 percent of firms) and commercial banks (10 percent). Equipment loans were also obtained mainly from these sources, with finance companies used somewhat less than commercial banks. Leases were obtained mainly from finance and leasing companies. However, less than 9 percent of businesses reported a capital lease in 2003. Finally, family and individuals were the source of "other" loans for 5 percent of firms, and commercial banks were the source of these loans for about 3 percent of firms.

In sum, commercial banks were the dominant source of lines, loans, and leases. By credit type, commercial banks were the dominant source of lines, mortgages, and equipment loans; and they were the second most important source for vehicle and other loans. The only credit type for which commercial banks were not an important source was capital leases. ${ }^{33}$

\section{Financial Management Services}

Commercial banks were the dominant supplier of financial management services, providing almost half of small businesses with those services in 2003 (table 10). Brokerages and credit card processing firms, tied as the second most common source of financial management services, were each used by 13 percent of firms. By individual service, commercial banks were the dominant supplier of transaction services (used by 34 percent of firms), credit card processing (21 percent), cash services (6 percent), and credit services (4 percent). Brokerages were the most widely used source of trust and pension services (used by 10 percent of firms) and brokerage services ( 5 percent). Credit card processing firms, used by 13 percent of firms for credit and debit card processing, were the second most common supplier of these services.

\section{SUMMARY}

The 2003 Survey of Small Business Finances, the fourth in a series sponsored by the Federal Reserve Board, provides detailed information on the character-

33. Of the six types of credit, capital leases were used by the smallest percentage of firms (8.7 percent). istics of small businesses and on the types and sources of credit and other financial services they use. The preceding surveys covered the years 1987, 1993, and 1998. Although the discussion in this article is based on descriptive statistics, the data suggest interesting behavior patterns and differences in the use of credit by small businesses.

Straightforward comparisons reveal some similarities in the findings from the earlier surveys. In particular, commercial banks have remained the dominant supplier of financial services for small businesses; these services include checking and savings accounts, most forms of credit other than leases, and most financial management services other than brokerage services.

Comparisons also reveal some changes between surveys. The share of small businesses that are $\mathrm{S}$ corporations has risen at the expense of $\mathrm{C}$ corporations and proprietorships. Computer use, especially for Internet banking and online loan applications, increased markedly between 1998 and 2003. The payment of business expenses with credit cards, especially business credit cards, has grown substantially between surveys. The incidence of credit lines and vehicle loans has increased, whereas the incidence of capital leases declined somewhat. Since the 1987 survey, small businesses have increasingly used nondepository institutions to obtain some of their financial services. However, despite the growth in the use of nondepository sources-from 25 percent of firms in 1987 to 54 percent in 2003-commercial banks remained the dominant supplier of most financial services.

The 2003 survey also provides some new information unavailable in previous surveys. In particular, the survey collected demographic characteristics for up to three individual owners and thereby helped to refine the firm-level measurement of characteristics such as race, ethnicity, sex, and ownership concentration. In addition, the survey collected information on the use of nonstandard work arrangements by firms and about their use of credit and debit card processing.

Explaining the differences among firms in their use of financing and, more fundamentally, understanding the factors that affect small business financing require a rigorous analytical framework that accounts for the financial characteristics of borrowers and the markets in which they operate. Although the use of such a framework is beyond the scope of this article, the final survey data will provide considerable opportunities for more formal and complete analyses. 
A.1. Small businesses grouped by selected characteristics and distributed by selected characteristics of firms, 2003 survey Percent

\begin{tabular}{|c|c|c|c|c|c|c|c|c|c|c|c|c|c|c|c|c|}
\hline \multirow{2}{*}{ Characteristic } & \multirow{2}{*}{$\begin{array}{l}\text { All } \\
\text { firms }\end{array}$} & \multicolumn{4}{|c|}{ Majority owners } & \multicolumn{5}{|c|}{ Number of employees } & \multicolumn{2}{|c|}{$\begin{array}{l}\text { Years under } \\
\text { current } \\
\text { ownership }\end{array}$} & \multicolumn{2}{|c|}{$\begin{array}{l}\text { Urbanization } \\
\text { at main office }\end{array}$} & \multicolumn{2}{|c|}{$\begin{array}{l}\text { Organizational } \\
\text { form }\end{array}$} \\
\hline & & $\begin{array}{c}\text { Non- } \\
\text { white } \\
\text { or His- } \\
\text { panic }\end{array}$ & $\begin{array}{l}\text { Non- } \\
\text { His- } \\
\text { panic } \\
\text { white }\end{array}$ & $\begin{array}{l}\text { Male or } \\
\text { divided } \\
\text { equally } \\
\text { by sex }\end{array}$ & Female & $0-1$ & $2-4$ & $5-19$ & $20-49$ & $50-499$ & $0-9$ & $\begin{array}{l}10 \text { or } \\
\text { more }\end{array}$ & Urban & Rural & $\begin{array}{c}\text { Pro- } \\
\text { prietor- } \\
\text { ship }\end{array}$ & Other \\
\hline All firms $\ldots \ldots \ldots \ldots \ldots$ & 100 & 100 & 100 & 100 & 100 & 100 & 100 & 100 & 100 & 100 & 100 & 100 & 100 & 100 & 100 & 100 \\
\hline 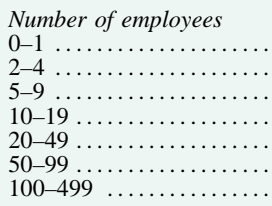 & $\begin{array}{r}20.6 \\
40.0 \\
20.2 \\
10.6 \\
6.0 \\
1.7 \\
1.0\end{array}$ & $\begin{array}{r}19.7 \\
46.1 \\
21.1 \\
5.7 \\
5.1 \\
1.4 \\
.8\end{array}$ & $\begin{array}{r}21.0 \\
39.3 \\
19.8 \\
11.2 \\
6.1 \\
1.7 \\
.9\end{array}$ & $\begin{array}{r}18.1 \\
40.2 \\
20.6 \\
11.7 \\
6.5 \\
1.8 \\
1.1\end{array}$ & $\begin{array}{r}29.5 \\
39.7 \\
18.9 \\
6.6 \\
4.0 \\
.9 \\
.4\end{array}$ & $\begin{array}{l}100 \\
\ldots \\
\ldots \\
\ldots \\
\ldots \\
\ldots \\
\ldots\end{array}$ & $\begin{array}{l}100 \\
\ldots \\
\ldots \\
\ldots \\
\ldots \\
\ldots\end{array}$ & $\begin{array}{l}\ldots \\
65.6 \\
34.4 \\
\ldots \\
\ldots \\
\ldots\end{array}$ & $\begin{array}{l}\ldots \\
\ldots \\
\ldots \\
\ldots \\
\ldots\end{array}$ & $\begin{array}{l}\ldots \\
\ldots \\
\ldots \\
\ldots \\
63.1 \\
37.0\end{array}$ & $\begin{array}{r}22.9 \\
41.1 \\
20.2 \\
9.6 \\
4.9 \\
1.0 \\
.4\end{array}$ & $\begin{array}{r}18.9 \\
39.2 \\
20.1 \\
11.3 \\
6.8 \\
2.2 \\
1.4\end{array}$ & $\begin{array}{r}20.7 \\
39.9 \\
20.3 \\
10.3 \\
5.9 \\
1.8 \\
1.1\end{array}$ & $\begin{array}{r}20.1 \\
40.4 \\
19.6 \\
11.5 \\
6.4 \\
1.3 \\
.8\end{array}$ & $\begin{array}{c}35.2 \\
46.7 \\
13.4 \\
3.7 \\
.9 \\
* \\
*\end{array}$ & $\begin{array}{r}8.9 \\
34.7 \\
25.6 \\
16.1 \\
10.1 \\
2.9 \\
1.8\end{array}$ \\
\hline 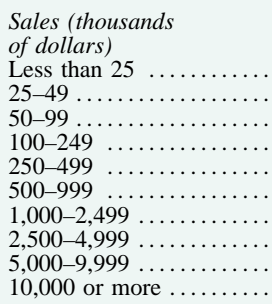 & $\begin{array}{r}14.6 \\
9.9 \\
11.6 \\
19.8 \\
14.3 \\
12.2 \\
10.0 \\
3.6 \\
2.3 \\
1.7\end{array}$ & $\begin{array}{r}20.0 \\
12.6 \\
16.4 \\
14.9 \\
14.1 \\
8.8 \\
7.5 \\
2.8 \\
1.8 \\
1.2\end{array}$ & $\begin{array}{r}14.0 \\
9.8 \\
11.0 \\
20.4 \\
14.5 \\
12.4 \\
10.3 \\
3.8 \\
2.1 \\
1.7\end{array}$ & $\begin{array}{r}11.2 \\
8.6 \\
10.7 \\
20.5 \\
15.5 \\
13.5 \\
11.4 \\
4.1 \\
2.7 \\
2.0\end{array}$ & $\begin{array}{r}26.6 \\
14.7 \\
14.9 \\
17.2 \\
10.3 \\
7.6 \\
5.6 \\
2.0 \\
.6 \\
.5\end{array}$ & $\begin{array}{c}37.3 \\
22.5 \\
17.5 \\
14.4 \\
4.5 \\
* \\
* \\
* \\
* \\
*\end{array}$ & $\begin{array}{r}15.5 \\
11.3 \\
16.8 \\
29.5 \\
16.5 \\
6.1 \\
3.0 \\
1.1 \\
* \\
*\end{array}$ & $\begin{array}{r}2.2 \\
2.3 \\
4.1 \\
16.0 \\
21.1 \\
27.1 \\
19.6 \\
4.9 \\
2.6 \\
*\end{array}$ & $\begin{array}{c}* \\
* \\
* \\
* \\
4.5 \\
14.6 \\
37.6 \\
20.7 \\
12.4 \\
5.9\end{array}$ & $\begin{array}{c}* \\
* \\
* \\
* \\
* \\
* \\
12.6 \\
14.5 \\
27.1 \\
43.4\end{array}$ & $\begin{array}{r}18.4 \\
11.4 \\
12.2 \\
19.7 \\
14.0 \\
11.4 \\
8.6 \\
2.4 \\
1.3 \\
.7\end{array}$ & $\begin{array}{r}11.8 \\
8.8 \\
11.1 \\
19.9 \\
14.5 \\
12.7 \\
11.1 \\
4.5 \\
3.0 \\
2.5\end{array}$ & $\begin{array}{r}14.3 \\
9.8 \\
11.1 \\
19.7 \\
14.5 \\
12.9 \\
10.0 \\
3.6 \\
2.3 \\
1.8\end{array}$ & $\begin{array}{r}15.6 \\
10.5 \\
13.3 \\
20.3 \\
13.5 \\
9.2 \\
10.3 \\
3.6 \\
2.2 \\
1.5\end{array}$ & $\begin{array}{r}24.9 \\
15.8 \\
18.5 \\
21.7 \\
9.8 \\
5.8 \\
2.5 \\
* \\
* \\
*\end{array}$ & $\begin{array}{r}6.3 \\
5.2 \\
6.1 \\
18.3 \\
17.8 \\
17.2 \\
16.1 \\
6.3 \\
3.7 \\
3.0\end{array}$ \\
\hline 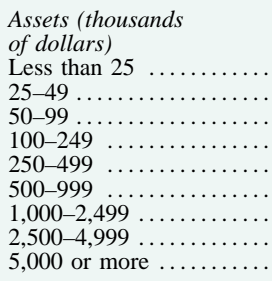 & $\begin{array}{r}31.3 \\
12.5 \\
13.5 \\
15.9 \\
10.0 \\
7.1 \\
5.8 \\
1.9 \\
2.0\end{array}$ & $\begin{array}{r}37.9 \\
14.4 \\
12.6 \\
15.0 \\
5.5 \\
5.0 \\
6.3 \\
2.8 \\
.6\end{array}$ & $\begin{array}{r}30.6 \\
12.4 \\
13.6 \\
15.8 \\
10.7 \\
7.4 \\
5.7 \\
1.7 \\
2.2\end{array}$ & $\begin{array}{r}27.0 \\
12.6 \\
13.5 \\
17.3 \\
11.0 \\
7.7 \\
6.6 \\
2.1 \\
2.3\end{array}$ & $\begin{array}{r}46.9 \\
12.4 \\
13.6 \\
11.0 \\
6.8 \\
4.8 \\
2.8 \\
1.0 \\
.7\end{array}$ & $\begin{array}{r}63.1 \\
13.9 \\
10.5 \\
6.5 \\
3.1 \\
* \\
* \\
* \\
*\end{array}$ & $\begin{array}{r}37.5 \\
16.3 \\
16.1 \\
15.8 \\
7.4 \\
3.8 \\
1.8 \\
* \\
*\end{array}$ & $\begin{array}{r}10.4 \\
9.2 \\
14.6 \\
23.5 \\
17.6 \\
12.8 \\
9.2 \\
1.5 \\
*\end{array}$ & $\begin{array}{r}* \\
* \\
5.3 \\
15.4 \\
14.3 \\
17.7 \\
25.8 \\
9.9 \\
4.8\end{array}$ & $\begin{array}{c}* \\
* \\
* \\
3.6 \\
6.8 \\
8.4 \\
20.7 \\
22.7 \\
35.4\end{array}$ & $\begin{array}{r}37.0 \\
13.4 \\
13.3 \\
15.3 \\
8.5 \\
5.8 \\
3.9 \\
1.2 \\
1.6\end{array}$ & $\begin{array}{r}27.1 \\
11.9 \\
13.6 \\
16.3 \\
11.2 \\
8.0 \\
7.2 \\
2.4 \\
2.4\end{array}$ & $\begin{array}{r}32.7 \\
12.4 \\
13.4 \\
15.4 \\
10.2 \\
6.3 \\
5.6 \\
2.0 \\
2.0\end{array}$ & $\begin{array}{r}26.1 \\
13.0 \\
13.6 \\
18.0 \\
9.6 \\
9.8 \\
6.5 \\
1.3 \\
2.1\end{array}$ & $\begin{array}{r}45.7 \\
14.0 \\
15.2 \\
13.2 \\
6.1 \\
2.9 \\
2.3 \\
* \\
*\end{array}$ & $\begin{array}{r}19.8 \\
11.3 \\
12.1 \\
18.1 \\
13.2 \\
10.4 \\
8.6 \\
3.0 \\
3.5\end{array}$ \\
\hline 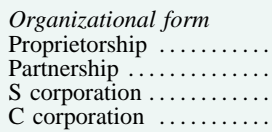 & $\begin{array}{r}44.5 \\
8.7 \\
31.0 \\
15.8\end{array}$ & $\begin{array}{r}47.8 \\
7.7 \\
25.5 \\
19.0\end{array}$ & $\begin{array}{r}44.6 \\
8.6 \\
31.9 \\
15.0\end{array}$ & $\begin{array}{r}41.3 \\
9.0 \\
32.9 \\
16.9\end{array}$ & $\begin{array}{r}56.3 \\
7.5 \\
24.8 \\
11.4\end{array}$ & $\begin{array}{c}76.0 \\
* \\
15.5 \\
5.8\end{array}$ & $\begin{array}{l}51.9 \\
10.9 \\
25.9 \\
11.3\end{array}$ & $\begin{array}{r}24.7 \\
9.9 \\
43.2 \\
22.3\end{array}$ & $\begin{array}{r}6.9 \\
9.7 \\
49.0 \\
34.4\end{array}$ & $\begin{array}{r}3.9 \\
7.2 \\
47.3 \\
41.7\end{array}$ & $\begin{array}{l}42.2 \\
12.3 \\
34.5 \\
11.0\end{array}$ & $\begin{array}{r}46.2 \\
6.0 \\
28.4 \\
19.3\end{array}$ & $\begin{array}{r}42.7 \\
8.5 \\
32.8 \\
16.0\end{array}$ & $\begin{array}{r}51.6 \\
9.6 \\
24.1 \\
14.8\end{array}$ & $\begin{array}{l}100 \\
\cdots \\
\cdots \\
\cdots\end{array}$ & $\begin{array}{l}15.7 \\
55.9 \\
28.4\end{array}$ \\
\hline 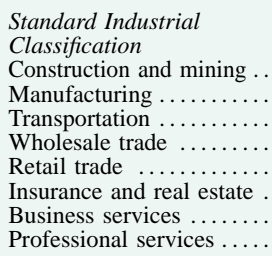 & $\begin{array}{r}11.8 \\
7.1 \\
3.8 \\
5.9 \\
18.4 \\
7.2 \\
25.1 \\
20.7\end{array}$ & $\begin{array}{r}5.7 \\
6.0 \\
2.6 \\
6.8 \\
21.1 \\
4.4 \\
31.6 \\
21.8\end{array}$ & $\begin{array}{r}12.8 \\
7.5 \\
4.0 \\
5.7 \\
18.1 \\
7.5 \\
24.2 \\
20.2\end{array}$ & $\begin{array}{r}13.7 \\
7.9 \\
4.3 \\
6.2 \\
18.1 \\
7.6 \\
23.4 \\
18.7\end{array}$ & $\begin{array}{r}5.2 \\
4.4 \\
1.8 \\
4.7 \\
19.5 \\
5.8 \\
31.3 \\
27.3\end{array}$ & $\begin{array}{r}13.1 \\
4.8 \\
4.5 \\
5.1 \\
10.7 \\
5.6 \\
31.1 \\
25.0\end{array}$ & $\begin{array}{r}10.5 \\
6.7 \\
3.2 \\
6.0 \\
16.9 \\
9.3 \\
26.2 \\
21.3\end{array}$ & $\begin{array}{r}12.1 \\
7.6 \\
4.0 \\
6.0 \\
23.2 \\
6.7 \\
22.5 \\
18.1\end{array}$ & $\begin{array}{r}15.0 \\
9.7 \\
2.6 \\
6.4 \\
30.9 \\
3.0 \\
15.0 \\
17.3\end{array}$ & $\begin{array}{r}10.9 \\
20.3 \\
7.4 \\
8.6 \\
19.4 \\
3.5 \\
15.4 \\
14.5\end{array}$ & $\begin{array}{r}10.6 \\
7.3 \\
3.8 \\
4.8 \\
21.8 \\
6.0 \\
27.7 \\
18.1\end{array}$ & $\begin{array}{r}12.7 \\
7.0 \\
3.8 \\
6.7 \\
15.9 \\
8.1 \\
23.2 \\
22.6\end{array}$ & $\begin{array}{r}10.4 \\
7.1 \\
3.3 \\
6.2 \\
18.1 \\
7.1 \\
24.9 \\
22.8\end{array}$ & $\begin{array}{r}17.3 \\
7.1 \\
5.3 \\
4.8 \\
19.7 \\
7.5 \\
25.7 \\
12.5\end{array}$ & $\begin{array}{r}12.0 \\
4.7 \\
3.6 \\
3.0 \\
18.0 \\
6.5 \\
29.9 \\
22.3\end{array}$ & $\begin{array}{r}11.7 \\
9.0 \\
3.9 \\
8.2 \\
18.7 \\
7.8 \\
21.3 \\
19.3\end{array}$ \\
\hline 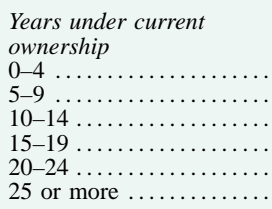 & $\begin{array}{l}20.6 \\
22.1 \\
16.0 \\
12.6 \\
10.9 \\
17.9\end{array}$ & $\begin{array}{r}33.4 \\
23.2 \\
13.2 \\
10.1 \\
9.1 \\
11.0\end{array}$ & $\begin{array}{l}18.5 \\
21.9 \\
16.5 \\
13.0 \\
11.1 \\
18.9\end{array}$ & $\begin{array}{l}20.0 \\
20.7 \\
15.6 \\
12.7 \\
11.4 \\
19.6\end{array}$ & $\begin{array}{r}23.1 \\
26.9 \\
17.1 \\
12.3 \\
8.9 \\
11.7\end{array}$ & $\begin{array}{l}26.7 \\
20.7 \\
17.3 \\
11.5 \\
11.0 \\
12.7\end{array}$ & $\begin{array}{l}21.5 \\
22.4 \\
16.2 \\
11.6 \\
10.1 \\
18.3\end{array}$ & $\begin{array}{l}18.0 \\
23.3 \\
14.9 \\
14.3 \\
11.6 \\
18.0\end{array}$ & $\begin{array}{r}13.4 \\
21.5 \\
15.9 \\
12.7 \\
9.9 \\
26.5\end{array}$ & $\begin{array}{r}6.6 \\
14.8 \\
15.9 \\
16.4 \\
14.2 \\
32.1\end{array}$ & $\begin{array}{l}\cdots \\
\cdots \\
\cdots \\
\cdots\end{array}$ & $\begin{array}{l}\ldots \\
27.9 \\
22.0 \\
18.9 \\
31.2\end{array}$ & $\begin{array}{l}22.1 \\
21.8 \\
15.8 \\
12.5 \\
11.2 \\
16.6\end{array}$ & $\begin{array}{r}15.0 \\
23.1 \\
16.7 \\
12.9 \\
9.5 \\
22.8\end{array}$ & $\begin{array}{l}20.6 \\
19.9 \\
16.7 \\
12.6 \\
12.2 \\
18.0\end{array}$ & $\begin{array}{r}20.6 \\
23.8 \\
15.4 \\
12.6 \\
9.7 \\
17.9\end{array}$ \\
\hline
\end{tabular}

\section{APPENDIX A: SURVEY METHODS}

The 2003 Survey of Small Business Finances was conducted in 2004 and 2005 for the Board of Governors of the Federal Reserve System by the National Opinion Research Center (NORC), a research organization at the University of Chicago. The survey covered a nationally representative sample of U.S. for-profit, nonfinancial, nonsubsidiary, nonagricul- tural, nongovernmental businesses with fewer than 500 employees that were in operation both at yearend 2003 and at the time of the interview. Most interviews took place between June 2004 and January $2005 . .^{34}$

34. Further details are in NORC (2005), "The 2003 Survey of Small Business Finances Methodology Report,' June, www.federalreserve. gov/pubs/oss/oss3/nssbftoc.htm. 


\section{A.1.-Continued}

Percent

\begin{tabular}{|c|c|c|c|c|c|c|c|c|c|c|c|c|c|c|c|c|}
\hline \multirow{2}{*}{ Characteristic } & \multirow{2}{*}{$\begin{array}{l}\text { All } \\
\text { firms }\end{array}$} & \multicolumn{4}{|c|}{ Majority owners } & \multicolumn{5}{|c|}{ Number of employees } & \multicolumn{2}{|c|}{$\begin{array}{l}\text { Years under } \\
\text { current } \\
\text { ownership }\end{array}$} & \multicolumn{2}{|c|}{$\begin{array}{l}\text { Urbanization } \\
\text { at main office }\end{array}$} & \multicolumn{2}{|c|}{$\begin{array}{l}\text { Organizational } \\
\text { form }\end{array}$} \\
\hline & & $\begin{array}{c}\text { Non- } \\
\text { white } \\
\text { or His- } \\
\text { panic }\end{array}$ & $\begin{array}{l}\text { Non- } \\
\text { His- } \\
\text { panic } \\
\text { white }\end{array}$ & $\begin{array}{c}\text { Male or } \\
\text { divided } \\
\text { equally } \\
\text { by sex }\end{array}$ & Female & $0-1$ & $2-4$ & $5-19$ & $20-49$ & $50-499$ & $0-9$ & $\begin{array}{l}10 \text { or } \\
\text { more }\end{array}$ & Urban & Rural & $\begin{array}{c}\text { Pro- } \\
\text { prietor- } \\
\text { ship }\end{array}$ & Other \\
\hline $\begin{array}{l}\text { Census area } \\
\text { of main office } \\
\text { Northeast } \ldots . . . . . \ldots \ldots \\
\quad \text { New England ........ } \\
\text { Middle Atlantic ..... }\end{array}$ & $\begin{array}{r}19.8 \\
6.0 \\
13.8\end{array}$ & $\begin{array}{c}14.9 \\
* \\
12.7\end{array}$ & $\begin{array}{r}20.5 \\
6.5 \\
14.0\end{array}$ & $\begin{array}{r}19.1 \\
5.6 \\
13.5\end{array}$ & $\begin{array}{r}22.1 \\
7.2 \\
14.9\end{array}$ & $\begin{array}{r}24.9 \\
7.6 \\
17.3\end{array}$ & $\begin{array}{r}19.1 \\
5.0 \\
14.1\end{array}$ & $\begin{array}{r}17.9 \\
6.1 \\
11.8\end{array}$ & $\begin{array}{r}18.3 \\
6.6 \\
11.7\end{array}$ & $\begin{array}{r}15.5 \\
4.4 \\
11.1\end{array}$ & $\begin{array}{r}18.3 \\
4.8 \\
13.5\end{array}$ & $\begin{array}{r}20.8 \\
6.8 \\
14.0\end{array}$ & $\begin{array}{r}21.5 \\
6.1 \\
15.4\end{array}$ & $\begin{array}{r}13.2 \\
5.6 \\
7.7\end{array}$ & $\begin{array}{r}20.5 \\
6.7 \\
13.9\end{array}$ & $\begin{array}{r}19.2 \\
5.4 \\
13.7\end{array}$ \\
\hline $\begin{array}{l}\text { Midwest ............ } \\
\text { East North Central } \\
\text { West North Central }\end{array}$ & $\begin{array}{r}21.1 \\
14.2 \\
6.9\end{array}$ & $\begin{array}{r}10.8 \\
8.4 \\
2.5\end{array}$ & $\begin{array}{r}22.5 \\
15.0 \\
7.5\end{array}$ & $\begin{array}{r}21.8 \\
14.8 \\
7.1\end{array}$ & $\begin{array}{r}18.3 \\
12.2 \\
6.1\end{array}$ & $\begin{array}{r}16.6 \\
12.2 \\
4.4\end{array}$ & $\begin{array}{r}21.4 \\
14.8 \\
6.6\end{array}$ & $\begin{array}{r}22.1 \\
13.4 \\
8.7\end{array}$ & $\begin{array}{r}27.8 \\
20.4 \\
7.4\end{array}$ & $\begin{array}{r}24.9 \\
15.7 \\
9.3\end{array}$ & $\begin{array}{r}19.1 \\
13.6 \\
5.5\end{array}$ & $\begin{array}{r}22.6 \\
14.7 \\
7.9\end{array}$ & $\begin{array}{r}18.7 \\
14.0 \\
4.7\end{array}$ & $\begin{array}{l}30.3 \\
15.1 \\
15.2\end{array}$ & $\begin{array}{r}19.9 \\
13.8 \\
6.0\end{array}$ & $\begin{array}{r}22.1 \\
14.5 \\
7.6\end{array}$ \\
\hline $\begin{array}{l}\text { South ...................... } \\
\text { South Atlantic ... } \\
\text { East South Central } \\
\text { West South Central . }\end{array}$ & $\begin{array}{r}34.7 \\
18.9 \\
5.3 \\
10.5\end{array}$ & $\begin{array}{r}44.7 \\
26.2 \\
6.2 \\
12.4\end{array}$ & $\begin{array}{r}33.6 \\
17.8 \\
5.3 \\
10.5\end{array}$ & $\begin{array}{r}34.5 \\
19.4 \\
5.1 \\
10.1\end{array}$ & $\begin{array}{r}35.4 \\
17.3 \\
5.9 \\
12.1\end{array}$ & $\begin{array}{r}31.6 \\
15.9 \\
5.4 \\
10.3\end{array}$ & $\begin{array}{r}35.7 \\
18.6 \\
6.0 \\
11.1\end{array}$ & $\begin{array}{r}36.9 \\
22.3 \\
4.3 \\
10.3\end{array}$ & $\begin{array}{r}29.7 \\
15.0 \\
5.7 \\
9.0\end{array}$ & $\begin{array}{r}30.6 \\
16.6 \\
4.7 \\
9.3\end{array}$ & $\begin{array}{r}39.0 \\
20.7 \\
6.1 \\
12.1\end{array}$ & $\begin{array}{r}31.6 \\
17.6 \\
4.7 \\
9.3\end{array}$ & $\begin{array}{r}34.6 \\
19.9 \\
3.9 \\
10.8\end{array}$ & $\begin{array}{r}35.1 \\
15.2 \\
10.6 \\
9.4\end{array}$ & $\begin{array}{r}32.6 \\
15.0 \\
6.3 \\
11.3\end{array}$ & $\begin{array}{r}36.5 \\
22.1 \\
4.5 \\
9.9\end{array}$ \\
\hline $\begin{array}{l}\text { West } \ldots \ldots \ldots \ldots \ldots \\
\quad \text { Mountain } \ldots \ldots \ldots \ldots \ldots \\
\text { Pacific } \ldots \ldots \ldots \ldots \ldots\end{array}$ & $\begin{array}{r}24.4 \\
7.6 \\
16.8\end{array}$ & $\begin{array}{r}29.6 \\
5.6 \\
24.0\end{array}$ & $\begin{array}{r}23.5 \\
8.0 \\
15.5\end{array}$ & $\begin{array}{r}24.5 \\
7.9 \\
16.6\end{array}$ & $\begin{array}{r}24.3 \\
6.7 \\
17.6\end{array}$ & $\begin{array}{r}26.9 \\
9.1 \\
17.8\end{array}$ & $\begin{array}{r}23.9 \\
6.3 \\
17.6\end{array}$ & $\begin{array}{r}23.1 \\
8.2 \\
14.9\end{array}$ & $\begin{array}{r}24.3 \\
9.2 \\
15.0\end{array}$ & $\begin{array}{r}28.9 \\
5.1 \\
23.8\end{array}$ & $\begin{array}{r}23.6 \\
7.5 \\
16.1\end{array}$ & $\begin{array}{r}25.0 \\
7.7 \\
17.3\end{array}$ & $\begin{array}{r}25.2 \\
6.5 \\
18.7\end{array}$ & $\begin{array}{r}21.4 \\
11.9 \\
9.5\end{array}$ & $\begin{array}{r}27.0 \\
6.9 \\
20.1\end{array}$ & $\begin{array}{r}22.3 \\
8.2 \\
14.1\end{array}$ \\
\hline $\begin{array}{l}\text { Urbanization } \\
\text { at main office } \\
\text { Urban .................. } \\
\text { Rural } \ldots \ldots \ldots \ldots \ldots \ldots \ldots\end{array}$ & $\begin{array}{l}79.4 \\
20.6\end{array}$ & $\begin{array}{l}89.6 \\
10.4\end{array}$ & $\begin{array}{l}77.6 \\
22.4\end{array}$ & $\begin{array}{l}79.2 \\
20.8\end{array}$ & $\begin{array}{l}80.2 \\
19.8\end{array}$ & $\begin{array}{l}79.9 \\
20.1\end{array}$ & $\begin{array}{l}79.2 \\
20.8\end{array}$ & $\begin{array}{l}79.1 \\
20.9\end{array}$ & $\begin{array}{l}77.8 \\
22.2\end{array}$ & $\begin{array}{l}84.5 \\
15.6\end{array}$ & $\begin{array}{l}81.5 \\
18.5\end{array}$ & $\begin{array}{l}77.8 \\
22.2\end{array}$ & $\begin{array}{l}100 \\
\ldots\end{array}$ & $\therefore 00$ & $\begin{array}{l}76.1 \\
23.9\end{array}$ & $\begin{array}{l}82.0 \\
18.0\end{array}$ \\
\hline $\begin{array}{l}\text { Number of offices } \\
\text { One } \ldots \ldots \ldots \ldots \ldots \ldots \ldots \\
\text { Two } \ldots \ldots \ldots \ldots \ldots \ldots \\
\text { Three or more } \ldots \ldots \ldots\end{array}$ & $\begin{array}{r}86.0 \\
9.4 \\
4.6\end{array}$ & $\begin{array}{r}86.6 \\
9.5 \\
3.9\end{array}$ & $\begin{array}{r}86.0 \\
9.5 \\
4.6\end{array}$ & $\begin{array}{r}85.2 \\
9.8 \\
5.0\end{array}$ & $\begin{array}{r}88.9 \\
8.1 \\
3.0\end{array}$ & $\begin{array}{l}93.5 \\
5.2 \\
*\end{array}$ & $\begin{array}{r}89.7 \\
8.3 \\
2.0\end{array}$ & $\begin{array}{r}82.8 \\
11.8 \\
5.4\end{array}$ & $\begin{array}{l}71.1 \\
15.1 \\
13.8\end{array}$ & $\begin{array}{l}41.0 \\
18.0 \\
41.1\end{array}$ & $\begin{array}{r}85.3 \\
10.8 \\
4.0\end{array}$ & $\begin{array}{r}86.5 \\
8.4 \\
5.1\end{array}$ & $\begin{array}{r}85.6 \\
9.9 \\
4.5\end{array}$ & $\begin{array}{r}87.3 \\
7.7 \\
5.0\end{array}$ & $\begin{array}{r}93.0 \\
5.8 \\
1.2\end{array}$ & $\begin{array}{r}80.3 \\
12.4 \\
7.4\end{array}$ \\
\hline $\begin{array}{l}\text { Sales area } \\
\text { Primarily within the } \\
\quad \text { United States .... } \\
\text { International or global }\end{array}$ & $\begin{array}{r}95.4 \\
4.6\end{array}$ & $\begin{array}{r}95.0 \\
5.0\end{array}$ & $\begin{array}{r}95.6 \\
4.4\end{array}$ & $\begin{array}{r}95.2 \\
4.8\end{array}$ & $\begin{array}{r}95.9 \\
4.1\end{array}$ & $\begin{array}{r}94.6 \\
5.4\end{array}$ & $\begin{array}{r}95.4 \\
4.6\end{array}$ & $\begin{array}{r}96.1 \\
4.0\end{array}$ & $\begin{array}{r}95.7 \\
4.3\end{array}$ & $\begin{array}{r}92.0 \\
8.0\end{array}$ & $\begin{array}{r}95.0 \\
5.0\end{array}$ & $\begin{array}{r}95.6 \\
4.4\end{array}$ & $\begin{array}{r}94.7 \\
5.3\end{array}$ & $\begin{array}{r}97.9 \\
2.1\end{array}$ & $\begin{array}{r}96.3 \\
3.7\end{array}$ & $\begin{array}{r}94.6 \\
5.4\end{array}$ \\
\hline $\begin{array}{l}\text { Management } \\
\text { By owner .............. } \\
\text { Hired .............. }\end{array}$ & $\begin{array}{r}94.3 \\
5.8\end{array}$ & $\begin{array}{r}92.8 \\
7.2\end{array}$ & $\begin{array}{r}94.6 \\
5.4\end{array}$ & $\begin{array}{r}94.6 \\
5.4\end{array}$ & $\begin{array}{r}93.0 \\
7.0\end{array}$ & $\begin{array}{l}99.1 \\
*\end{array}$ & $\begin{array}{r}96.2 \\
3.8\end{array}$ & $\begin{array}{r}91.5 \\
8.5\end{array}$ & $\begin{array}{l}85.9 \\
14.1\end{array}$ & $\begin{array}{l}76.9 \\
23.1\end{array}$ & $\begin{array}{r}94.7 \\
5.3\end{array}$ & $\begin{array}{r}93.9 \\
6.1\end{array}$ & $\begin{array}{r}94.0 \\
6.0\end{array}$ & $\begin{array}{r}95.2 \\
4.8\end{array}$ & $\begin{array}{r}97.4 \\
2.6\end{array}$ & $\begin{array}{r}91.7 \\
8.3\end{array}$ \\
\hline $\begin{array}{l}\text { Race, ethnicity, and } \\
\text { sex of majority owners } \\
\text { Nonwhite or Hispanic . } \\
\text { Non-Hispanic white ... }\end{array}$ & $\begin{array}{l}13.1 \\
86.6\end{array}$ & $\begin{array}{r}100.0 \\
6.3\end{array}$ & $\begin{array}{r}1.0 \\
100.0\end{array}$ & $\begin{array}{l}12.3 \\
86.9\end{array}$ & $\begin{array}{l}15.7 \\
85.8\end{array}$ & $\begin{array}{l}12.5 \\
87.9\end{array}$ & $\begin{array}{l}15.0 \\
85.0\end{array}$ & $\begin{array}{l}11.4 \\
87.3\end{array}$ & $\begin{array}{l}11.3 \\
89.0\end{array}$ & $\begin{array}{l}11.5 \\
88.8\end{array}$ & $\begin{array}{l}17.3 \\
82.0\end{array}$ & $\begin{array}{r}9.9 \\
90.1\end{array}$ & $\begin{array}{l}14.7 \\
84.6\end{array}$ & $\begin{array}{r}6.6 \\
94.3\end{array}$ & $\begin{array}{l}14.0 \\
86.4\end{array}$ & $\begin{array}{l}12.3 \\
86.8\end{array}$ \\
\hline $\begin{array}{l}\text { White } \ldots \ldots \ldots \ldots \ldots \\
\text { Black } \ldots \ldots \ldots \ldots \ldots \ldots \\
\text { Asian, Native Hawaiian, } \\
\text { or other Pacific } \\
\text { Islander ........... }\end{array}$ & $\begin{array}{r}91.0 \\
3.7\end{array}$ & $\begin{array}{l}35.0 \\
28.1\end{array}$ & $\begin{array}{c}100.0 \\
*\end{array}$ & $\begin{array}{r}91.4 \\
3.2\end{array}$ & $\begin{array}{r}89.8 \\
5.5\end{array}$ & $\begin{array}{r}91.9 \\
3.5\end{array}$ & $\begin{array}{r}90.0 \\
4.6\end{array}$ & $\begin{array}{r}90.9 \\
3.3\end{array}$ & $\begin{array}{c}94.4 \\
*\end{array}$ & $\begin{array}{c}92.2 \\
*\end{array}$ & $\begin{array}{r}87.9 \\
4.8\end{array}$ & $\begin{array}{r}93.3 \\
2.8\end{array}$ & $\begin{array}{r}89.6 \\
4.3\end{array}$ & $\begin{array}{c}96.3 \\
*\end{array}$ & $\begin{array}{r}90.7 \\
5.1\end{array}$ & $\begin{array}{r}91.2 \\
2.5\end{array}$ \\
\hline $\begin{array}{c}\text { American Indian or } \\
\text { Alaska Native }\end{array}$ & 1.3 & 10.3 & .7 & 1.1 & 2.2 & $*$ & 1.6 & $*$ & $*$ & $*$ & 1.7 & 1.1 & 1.2 & 1.9 & 1.7 & 1.1 \\
\hline $\begin{array}{l}\text { Hispanic ........... } \\
\text { Non-Hispanic } \ldots .\end{array}$ & $\begin{array}{r}4.2 \\
95.8\end{array}$ & $\begin{array}{l}31.8 \\
68.2\end{array}$ & 100.0 & $\begin{array}{r}4.1 \\
95.9\end{array}$ & $\begin{array}{r}4.3 \\
95.7\end{array}$ & $\begin{array}{r}4.0 \\
96.1\end{array}$ & $\begin{array}{r}4.8 \\
95.3\end{array}$ & $\begin{array}{r}3.3 \\
96.7\end{array}$ & $\begin{array}{r}5.6 \\
94.5\end{array}$ & $\begin{array}{r}3.5 \\
96.5\end{array}$ & $\begin{array}{r}5.2 \\
94.8\end{array}$ & $\begin{array}{r}3.4 \\
96.6\end{array}$ & $\begin{array}{r}4.7 \\
95.3\end{array}$ & $\begin{array}{r}2.2 \\
97.8\end{array}$ & $\begin{array}{r}4.5 \\
95.5\end{array}$ & $\begin{array}{r}3.9 \\
96.1\end{array}$ \\
\hline $\begin{array}{l}\text { Female .............. } \\
\text { Male .............. } \\
\text { Ownership divided } \\
\text { equally by sex }\end{array}$ & $\begin{array}{l}22.4 \\
64.8 \\
\\
12.8\end{array}$ & $\begin{array}{r}26.8 \\
65.1 \\
\\
8.1\end{array}$ & $\begin{array}{l}22.2 \\
65.3\end{array}$ & $\begin{array}{r}83.5 \\
16.5\end{array}$ & $\begin{array}{c}100 \\
\ldots\end{array}$ & $\begin{array}{l}32.0 \\
62.2\end{array}$ & $\begin{array}{l}22.1 \\
62.6\end{array}$ & $\begin{array}{l}18.5 \\
66.3 \\
15.2\end{array}$ & $\begin{array}{r}15.0 \\
75.4 \\
\\
9.6\end{array}$ & $\begin{array}{l}11.5 \\
80.5\end{array}$ & $\begin{array}{l}26.2 \\
60.7\end{array}$ & $\begin{array}{l}19.5 \\
67.9\end{array}$ & $\begin{array}{l}22.6 \\
65.3\end{array}$ & $\begin{array}{l}21.5 \\
62.9\end{array}$ & $\begin{array}{l}28.2 \\
62.4\end{array}$ & $\begin{array}{l}17.7 \\
66.8\end{array}$ \\
\hline
\end{tabular}

Note: For definition of sales areas, refer to table 2, note 3.

* Fewer than fifteen observations.

... Not applicable.

The sample was drawn from the Dun \& Bradstreet Market Identifier (DMI) file. ${ }^{35}$ The DMI file is broadly representative of all businesses in the United States (though it may underrepresent the newest and smallest businesses). It has been estimated that the

35. Dun's Marketing Service, Dun \& Bradstreet, Inc.
Dun \& Bradstreet database covers approximately 93 percent of full-time business activity. ${ }^{36}$

Entities known to be ineligible for the survey (such as firms with 500 or more employees, branches, subsidiaries, and firms in certain industries) were removed from the DMI file, and then the DMI file

36. Bruce D. Phillips and Bruce A. Kirchhoff, "Formation, Growth, and Survival: Small Firm Dynamics in the U.S. Economy," Small Business Economics, vol. 1 (March), pp. 65-74. 
was sampled according to a stratified systematic design. The design consisted of seventy-two strata defined by the cross-classification of firm size by number of employees (less than 20, 20-49, 50-99, and 100-499), Census division, and urban or rural status. Each stratum was sorted by Standard Industrial Classification (SIC) code to help ensure proportionate industry coverage. Larger small businesses (those with 20 or more employees), which account for a small proportion of the target population, were oversampled to ensure a large enough sample to permit comparisons with smaller small businesses.

A sample of 37,600 firms (representing nearly 9.7 million enterprises) was initially selected for a brief, computer-assisted telephone interview. The purpose of this screening interview was to verify that the firm was eligible for inclusion in the sample, to confirm contact information, and to secure cooperation for the main interview. In advance of the screening interview, selected firms received a brochure describing the survey. The average screening interview itself took less than eleven minutes, and the average total time per screening interview (which included establishing contact with firm owners, setting appointments, and calling back to complete the screening interview) was about one hour. Of these 37,600 firms, 23,798 were selected for the screening interview, 14,061 were actually screened, and 9,687 were determined to be eligible. The weighted response rate on the screening interview was 62 percent. ${ }^{37}$

In the second stage, the main survey, also a computer-assisted telephone interview, was attempted with all 9,687 firms determined to be eligible during the screening stage. ${ }^{38}$ Within a couple of weeks of completing the screening interview, firms received a customized worksheet to help the owners collect and organize their records in preparation for the main interview. The worksheet requested financial data for the firm, information about the financial services used by the firm, and the sources of those services. The worksheets were customized according to the firm's legal organizational form and directed respondents to the appropriate lines on their tax forms. ${ }^{39}$ When

37. Details on response rates are in NORC (2005), "The 2003 Survey of Small Business Finances Methodology Report."

38. During the main interview, a few additional firms were determined to be ineligible. In most of these cases, screening interviews had been completed by someone other than an owner.

39. At the end of the main interview, respondents were asked to return their worksheets or other records (tax files, financial reports) in a self-addressed stamped envelope. About one-third of the participating firms returned completed worksheets or other records. The preparation of the worksheets helped respondents prepare for the interview, and the returned worksheets often helped resolve discrepancies or supply items missing after the main interview. interviewing was finished, 4,240 eligible firms, representing 6.3 million businesses, had completed interviews-a completion rate of 52.4 percent for the main interview. The weighted response rate for screening and main interviews combined was 32.4 percent. ${ }^{40}$

The actual main telephone interview took an average of about fifty-five minutes, and the total time (which included establishing contact, setting appointments, and so on) averaged more than three hours per completed case. Typically, respondents that started the main interview and got through the first few questions ended up completing the interview.

The following categories of information were collected from each firm:

- the demographics of the firm and of the owners with the largest shares - up to three owners per firm

- the firm's use of financial services and the sources providing the services

- the most recent applications for credit by the firm in the past three years

- the firm's balance sheet and income data

- the recent credit history of the firm and its owners

With the exception of the income statement, balance sheet, and most recent credit applications, the data were collected as of the date of the interview. ${ }^{41}$

A public-use version of the data set and a user's manual will be posted on the Federal Reserve Board's website, at www.federalreserve.gov/pubs/oss/oss3/ nsbftoc.htm.

\section{APPENDIX B: RACE, ETHNICITY, AND BUSINESS OWNERSHIP}

In contrast to earlier years, the most recent SSBF survey and the most recent data released by the U.S. Census Bureau differ in the estimated share of all

40. The response rate for the $1998 \mathrm{SSBF}$ was 33 percent. One of the goals of the 2003 survey was to improve response rates. To this end, the 2003 survey offered incentives for completing the main interview, shortened the time between the screening interview and the main interview to an average of two weeks or less, sent worksheets to potential respondents using next-day delivery, added an automated procedure for looking up institutions during the main interview to reduce the amount of time needed to identify branch locations and obtain branch addresses, and carefully reviewed and streamlined the questionnaire. These efforts are believed to have prevented the 2003 response rate from declining from its 1998 level.

41. To ease reporting burden and to ensure some consistency across firms, balance sheet and income items were tied to the tax forms that most firms are required to file each fiscal year. Because not all fiscal years end on December 31, and because it takes time for businesses to organize records and prepare tax forms, firms were asked to report balance sheet and income data for the firm's fiscal year that ended between May 1, 2003, and April 30, 2004. 
businesses that were owned by Asians, by blacks, and by Hispanics. ${ }^{42}$ In the Census Bureau's Survey of Business Owners (SBO), the ownership rates of these minority groups rose between the 1997 and 2002 surveys. However, the SSBF estimates indicate that, between 1998 and 2003, rates of ownership among Asians and blacks were essentially unchanged, whereas rates of Hispanic ownership declined (table B.1). ${ }^{43}$

These differences can largely be explained by three factors:

- First, the lists of businesses from which the two surveys draw their samples (the sample frames) differ in ways that are sensitive to any disproportionate change in minority ownership rates. Such disproportionate changes were seen in the 2002-03 period but not in the 1997-98 period.

- Second, to improve the uniformity of its financial data, the 2003 SSBF lengthened the time that a firm would have to be in business to be eligible for the survey. This change would tend to cause a decrease in the observed rate of minority ownership in the SSBF relative to the SBO because minority-owned firms tend to have shorter life spans than nonminority-owned firms.

- Third, unlike the 1998 SSBF and the two SBO surveys, the 2003 SSBF did not oversample minority-owned businesses. Although that is a notable change in methodology, the specific effects of the change are unknown.

Taken together, these three factors suggest that the difference in minority ownership rates between the 2003 SSBF and the 2002 SBO stem from the interac-

42. Rates of ownership by American Indians and Alaska Natives were close in the two surveys and are thus not treated in this appendix. In the 1997 SBO, the Native Hawaiian category did not appear separately; in that survey, the category was "Asian and Pacific Islander." As in the rest of this article, "Asian" used in reference to the SSBF refers to individuals who are Asian, Native Hawaiian, or Pacific Islander.

43. The 1998 SSBF covered 3,561 firms, of which 214 were Asian-owned, 273 were black-owned, and 260 were Hispanic-owned. The corresponding ownership numbers for the 4,240 firms in the 2003 SSBF were 170,119 , and 149 respectively. Reported percentages are weighted to adjust for sample design and nonresponse. Because the samples in each survey were drawn from different populations and the Census Bureau does not provide standard errors, the point estimates are not directly comparable across surveys. For this appendix, estimate $B$ is considered statistically different from estimate $A$ if it does not lie within the 95 percent confidence interval of $A$. For example, in the 2003 SSBF, the percentage of firms that were owned by blacks was estimated to be 3.7 percent. The 95 percent confidence interval for that estimate is from 2.9 percent to 4.5 percent-calculated as $3.7 \pm(1.96 \mathrm{x}$ 0.4) (see table B.1). Because the 2002 SBO estimate of 5.2 percent falls outside this range, the difference between these two estimates is statistically significant.
B.1. Rates of business ownership, by selected race and ethnicity of owners, 1997 and 2002 SBO and 1998 and 2003 SSBF

Percent

\begin{tabular}{|c|c|c|c|c|}
\hline \multirow{2}{*}{ Race or ethnicity } & \multicolumn{2}{|c|}{ SBO } & \multicolumn{2}{|c|}{ SSBF } \\
\hline & 1997 & 2002 & 1998 & 2003 \\
\hline Asian $^{1}$ & 4.4 & 5.0 & $\begin{array}{c}4.4 \\
(.3)\end{array}$ & $\begin{array}{c}4.2 \\
(.4)\end{array}$ \\
\hline Black & 4.0 & 5.2 & $\begin{array}{c}4.1 \\
(.3)\end{array}$ & $\begin{array}{l}3.7 \\
(.4)\end{array}$ \\
\hline Hispanic & 5.8 & 6.9 & $\begin{array}{l}5.6 \\
(.4)\end{array}$ & $\begin{array}{c}4.2 \\
(.4)\end{array}$ \\
\hline
\end{tabular}

NoTE: Numbers in parentheses are standard errors.

1. For definition of this term here and in the following tables, refer to text note 42.

SourcE: Here and in the following tables, data for the Survey of Business Owners (SBO) are from total numbers of businesses, 1997 and 2002 surveys, www.census.gov/csd/sbo.

tion of survey structure with changes specific to the periods in question. Such complex interactions highlight the need to treat comparisons of results from the SSBF and SBO-indeed, between any two surveyswith care.

\section{Differences in Universes}

Perhaps the most important reason that the estimates of minority ownership rates from the most recent SSBF and SBO may have diverged is the difference in the promptness with which the surveys' sample frames pick up new and small businesses, together with some particular dynamics of business ownership in the periods between 1997 and 2003.

For both the 1997 and 2002 surveys, the SBO sample was drawn from economic census reports and from a list of firms compiled from federal tax returns filed by businesses. ${ }^{44}$ Because the list used by the Census Bureau is drawn in part from restricted sources and is not available to the Federal Reserve Board, the SSBF sample must be drawn from another source. The Dun \& Bradstreet Market Identifier File (DMI), thought to be the best publicly available listing of current businesses in the United States, is the source for the 1998 and 2003 SSBF samples. ${ }^{45}$ The DMI file is updated using information from new

44. The Census Bureau obtains electronic versions of the following forms submitted by businesses to the Internal Revenue Service: Form 1040, Schedule C (individual proprietorship or self-employed person); Form 1065 (partnership); all Form 1120 corporation tax forms; and Form 941 (Employer's Quarterly Federal Tax Return).

45. The Small Business Administration (The State of Small Business: A Report of the President, U.S. Small Business Administration, 1988) estimates that the DMI frame represented approximately 93 percent of private employment in 1987. No updated estimate of the extent of DMI coverage is available, but its coverage is likely to have improved (or at least held steady) with improved information technology. For example, in the early 1990s, the DMI frame began including 
B.2. Rates of business ownership, by selected race and ethnicity of owners and presence of paid employees, 1997 and 2002 SBO

Percent

\begin{tabular}{c|c|c|c|c}
\hline \multirow{2}{*}{ Race or ethnicity } & \multicolumn{2}{|c|}{ No paid employees } & \multicolumn{2}{c}{ Paid employees } \\
\cline { 2 - 5 } & 1997 & 2002 & 1997 & 2002 \\
\hline Asian _............ & 4.0 & 4.6 & 5.5 & 5.9 \\
Black _......... & 4.7 & 6.3 & 1.8 & 1.7 \\
Hispanic ........ & 6.4 & 7.9 & 4.0 & 3.6 \\
\hline
\end{tabular}

credit applications; in-person and telephone interviews; county, state, and federal government sources; business trade-tape exchange programs; and thirdparty sources such as listings in the Yellow Pages. Given that the newest and smallest firms often do not apply for new credit, advertise in the Yellow Pages, or incorporate, it is likely that the DMI frame underrepresents the smallest and newest firms. ${ }^{46}$

If the rates of minority ownership are higher for the firms missing from the DMI file than for those listed, we would expect to see lower rates of minority ownership in the SSBF than in the SBO. The SBO does not provide information on the ownership of the firm by firm age, so we are unable to say anything about the part of the DMI file that is likely to be missing because the firm is too new. However, the SBO does provide information according to whether the firm has paid employees and, if it does, according to the number of employees. Non-employer firms and firms with few paid employees are less likely to be represented on the DMI file than the employer firms, especially employer firms with a large number of employees.

\section{Business Ownership Rates from the SBO}

Among the firms represented by the 2002 SBO sample, less than one-fourth had any paid employees, a fraction consistent with the 1997 SBO. The SBO estimates indicate that ownership rates for blacks and Hispanics were much lower among firms with paid employees (table B.2). They also indicate that ownership rates among blacks and Hispanics grew only among firms with no paid employees. Among firms with paid employees, the rate of ownership among blacks remained roughly constant, and the rate among Hispanics showed a slight decline.

all firms in the business listings of telephone directories, such as the Yellow Pages.

46. An estimate of the coverage of the DMI file is in David A. Marker and W. Sherman Edwards (1997), "Quality of the DMI File as a Business Sampling Frame," in proceedings of the Survey Research Methods Section, American Statistical Association, pp. 21-30, www.amstat.org/sections/SRMS/proceedings.
B.3. Rates of ownership of businesses with paid employees, by selected race and ethnicity of owners and number of paid employees, 1997 and 2002 SBO Percent

\begin{tabular}{l|c|c|c|c|c|c}
\hline \multirow{2}{*}{$\begin{array}{c}\text { Number of } \\
\text { paid employees }\end{array}$} & \multicolumn{2}{|c|}{ Asian } & \multicolumn{2}{c|}{ Black } & \multicolumn{2}{c}{ Hispanic } \\
\cline { 2 - 7 } & 1997 & 2002 & 1997 & 2002 & 1997 & 2002 \\
\hline $1-4 \ldots \ldots \ldots \ldots$. & 5.7 & 6.2 & 1.9 & 1.8 & 4.3 & 3.8 \\
$5-9 \ldots \ldots \ldots \ldots$. & 5.6 & 5.8 & 1.5 & 1.5 & 3.9 & 3.3 \\
$10-19 \ldots \ldots \ldots \ldots$. & 4.5 & 5.2 & 1.3 & 1.3 & 3.5 & 3.2 \\
$20-49 \ldots \ldots \ldots \ldots$ & 4.3 & 4.1 & 1.2 & 1.2 & 2.6 & 2.5 \\
$50-99 \ldots \ldots \ldots \ldots$ & 3.5 & 2.9 & 1.3 & 1.1 & 2.7 & 2.4 \\
$100-499 \ldots \ldots \ldots$ & 2.5 & 2.2 & 1.0 & 1.1 & 1.3 & 1.6 \\
MEMO & & & & & & \\
All firms ......... & 5.5 & 5.9 & 1.8 & 1.7 & 4.0 & 3.6 \\
\hline
\end{tabular}

Asian ownership rates were higher for firms with paid employees than for those without paid employees, and Asian ownership rates grew somewhat for both these types of firms. However, Asian ownership rates were highest for the smallest firms; indeed, the growth between the 1997 and 2002 SBO was limited to firms with fewer than twenty paid employees (table B.3). ${ }^{47}$

\section{Composition of Self-Employment from the CPS}

Data from the March Current Population Survey (CPS) provide further details on the changes in business ownership composition in the periods between the 1997 and 2002 SBO and the 1998 and 2003 SSBF (table B.4). Although the CPS data are not intended to measure business ownership, they do provide information on self-employment, with a breakdown by business incorporation status. ${ }^{48} \mathrm{We}$ would expect new unincorporated businesses to be picked up less quickly in the DMI file than incorporated businesses for the reasons noted above. Changes in the make-up of self-employed individuals in unincorporated businesses are thus likely to be observed only with a lag in the DMI file. However, if the change is proportionate by race and ethnicity, the lag should not affect estimates of minority ownership rates.

47. Minority ownership rates by the number of employees are not available for firms without paid employees, as the numbers of unpaid employees are not collected.

48. Estimates of business ownership rates based on self-employment will not be exactly comparable to actual business ownership rates because the unit of observation is the individual rather than the firm. Counting this way will miss all individuals who do not actively work in their firms and overcount firms that are jointly owned; no information is available on the effect this is likely to have on estimates of minority ownership. Estimates are based on the "Class of Worker" variables for the main and second job; respondents were counted as self-employed if they reported self-employment for either job. From 1994 to 2003, between 10 percent and 12 percent of the employed population reported being self-employed in either their main or second job. 
B.4. Share of self-employed population that is of selected race and ethnicity, by status of business incorporation, Current Population Survey, 1994-2003 Percent

\begin{tabular}{|c|c|c|c|c|c|c|c|c|c|}
\hline \multirow{2}{*}{ Year } & \multicolumn{3}{|c|}{ All businesses } & \multicolumn{3}{|c|}{ Incorporated businesses } & \multicolumn{3}{|c|}{ Unincorporated businesses } \\
\hline & Asian & Black & Hispanic & Asian & Black & Hispanic & Asian & Black & Hispanic \\
\hline 1994. & $\begin{array}{l}2.8 \\
(.2)\end{array}$ & $\begin{array}{c}4.4 \\
(.3)\end{array}$ & $\begin{array}{l}5.3 \\
(.3)\end{array}$ & $\begin{array}{l}3.6 \\
(.4)\end{array}$ & $\begin{array}{l}2.9 \\
(.4)\end{array}$ & $\begin{array}{l}4.0 \\
(.5)\end{array}$ & $\begin{array}{l}2.4 \\
(.2)\end{array}$ & $\begin{array}{l}4.9 \\
(.3)\end{array}$ & $\begin{array}{l}5.8 \\
(.4)\end{array}$ \\
\hline 1995 . & $\begin{array}{l}2.5 \\
(.2)\end{array}$ & $\begin{array}{l}4.1 \\
(.3)\end{array}$ & $\begin{array}{l}4.7 \\
(.3)\end{array}$ & $\begin{array}{l}3.4 \\
(.4)\end{array}$ & $\begin{array}{l}2.6 \\
(.4)\end{array}$ & $\begin{array}{l}4.0 \\
(.5)\end{array}$ & $\begin{array}{l}2.1 \\
(.2)\end{array}$ & $\begin{array}{l}4.7 \\
(.3)\end{array}$ & $\begin{array}{l}5.0 \\
(.4)\end{array}$ \\
\hline $1996 \ldots$ & $\begin{array}{l}3.3 \\
(.2)\end{array}$ & $\begin{array}{l}4.4 \\
(.3)\end{array}$ & $\begin{array}{l}5.5 \\
(.3)\end{array}$ & $\begin{array}{l}4.5 \\
(.6)\end{array}$ & $\begin{array}{l}2.6 \\
(.5)\end{array}$ & $\begin{array}{l}3.2 \\
(.5)\end{array}$ & $\begin{array}{l}2.8 \\
(.3)\end{array}$ & $\begin{array}{l}5.0 \\
(.4)\end{array}$ & $\begin{array}{l}6.3 \\
(.4)\end{array}$ \\
\hline $1997 \ldots$ & $\begin{array}{l}4.0 \\
(.3)\end{array}$ & $\begin{array}{l}4.3 \\
(.3)\end{array}$ & $\begin{array}{l}5.6 \\
(.3)\end{array}$ & $\begin{array}{l}4.6 \\
(.5)\end{array}$ & $\begin{array}{l}2.5 \\
(.4)\end{array}$ & $\begin{array}{l}3.1 \\
(.5)\end{array}$ & $\begin{array}{l}3.8 \\
(.3)\end{array}$ & $\begin{array}{l}5.0 \\
(.4)\end{array}$ & $\begin{array}{l}6.6 \\
(.4)\end{array}$ \\
\hline 1998 & $\begin{array}{l}3.9 \\
(.3)\end{array}$ & $\begin{array}{l}4.4 \\
(.3)\end{array}$ & $\begin{array}{l}5.5 \\
(.3)\end{array}$ & $\begin{array}{l}4.3 \\
(.5)\end{array}$ & $\begin{array}{l}2.3 \\
(.4)\end{array}$ & $\begin{array}{l}3.3 \\
(.5)\end{array}$ & $\begin{array}{l}3.7 \\
(.3)\end{array}$ & $\begin{array}{l}5.2 \\
(.4)\end{array}$ & $\begin{array}{l}6.4 \\
(.4)\end{array}$ \\
\hline 1999 & $\begin{array}{l}4.3 \\
(.3)\end{array}$ & $\begin{array}{l}5.1 \\
(.3)\end{array}$ & $\begin{array}{l}6.4 \\
(.4)\end{array}$ & $\begin{array}{l}4.9 \\
(.5)\end{array}$ & $\begin{array}{l}3.9 \\
(.5)\end{array}$ & $\begin{array}{l}3.4 \\
(.5)\end{array}$ & $\begin{array}{l}4.1 \\
(.3)\end{array}$ & $\begin{array}{l}5.6 \\
(.4)\end{array}$ & $\begin{array}{l}7.5 \\
(.5)\end{array}$ \\
\hline $2000 \ldots$ & $\begin{array}{l}3.8 \\
(.3)\end{array}$ & $\begin{array}{l}5.8 \\
(.4)\end{array}$ & $\begin{array}{l}5.9 \\
(.3)\end{array}$ & $\begin{array}{l}4.4 \\
(.5)\end{array}$ & $\begin{array}{l}4.3 \\
(.6)\end{array}$ & $\begin{array}{l}4.7 \\
(.5)\end{array}$ & $\begin{array}{l}3.5 \\
(.3)\end{array}$ & $\begin{array}{l}6.5 \\
(.4)\end{array}$ & $\begin{array}{l}6.4 \\
(.4)\end{array}$ \\
\hline $2001 \ldots$ & $\begin{array}{l}4.4 \\
(.3)\end{array}$ & $\begin{array}{l}5.2 \\
(.3)\end{array}$ & $\begin{array}{l}6.3 \\
(.3)\end{array}$ & $\begin{array}{l}4.6 \\
(.5)\end{array}$ & $\begin{array}{l}4.0 \\
(.5)\end{array}$ & $\begin{array}{l}4.8 \\
(.5)\end{array}$ & $\begin{array}{l}4.3 \\
(.4)\end{array}$ & $\begin{array}{l}5.7 \\
(.4)\end{array}$ & $\begin{array}{l}6.8 \\
(.4)\end{array}$ \\
\hline $2002 \ldots$ & $\begin{array}{l}3.5 \\
(.3)\end{array}$ & $\begin{array}{l}5.6 \\
(.3)\end{array}$ & $\begin{array}{l}6.7 \\
(.4)\end{array}$ & $\begin{array}{l}4.0 \\
(.5)\end{array}$ & $\begin{array}{c}3.4 \\
(.4)\end{array}$ & $\begin{array}{l}3.5 \\
(.4)\end{array}$ & $\begin{array}{l}3.3 \\
(.3)\end{array}$ & $\begin{array}{l}6.6 \\
(.4)\end{array}$ & $\begin{array}{l}8.2 \\
(.5)\end{array}$ \\
\hline 2003 & $\begin{array}{l}4.5 \\
(.3)\end{array}$ & $\begin{array}{l}5.4 \\
(.3)\end{array}$ & $\begin{array}{l}7.7 \\
(.4)\end{array}$ & $\begin{array}{l}5.4 \\
(.5)\end{array}$ & $\begin{array}{l}4.2 \\
(.5)\end{array}$ & $\begin{array}{l}4.0 \\
(.5)\end{array}$ & $\begin{array}{l}4.1 \\
(.4)\end{array}$ & $\begin{array}{l}6.0 \\
(.4)\end{array}$ & $\begin{array}{l}9.4 \\
(.5)\end{array}$ \\
\hline
\end{tabular}

NoTE: Numbers in parentheses are standard errors.

SourCE: Calculated from Current Population Survey for March of each year, www.bls.census.gov/cps/cpsbasic.htm.

The data do indicate disproportionate changes in the composition of the self-employed in the 2002-03 period. Between 1997 and 1998, the proportions of the self-employed population that were Asian, black, and Hispanic were essentially unchanged. In particular, the proportions of self-employed persons in unincorporated businesses who were Asian, black, or Hispanic remained constant. This constancy would imply that, despite the lag with which the smallest new businesses are likely to appear in the DMI file, the estimated rates of minority ownership from a sample drawn from the DMI file during that period should be very close to the actual rates. Indeed, the rates of minority ownership in the $1998 \mathrm{SSBF}$ and the 1997 SBO were both very close to the 1998 estimated overall self-employment proportions calculated from the CPS.

Between 2002 and 2003, however, the proportion of self-employed individuals who were Asian rose about 1 percentage point, the proportion that was Hispanic also rose about 1 percentage point, and the share that was black changed little. Moreover, the proportions of self-employed individuals in unincorporated businesses who were Asian or Hispanic also rose between these years. Thus, unlike in the 1997-98 period, the 2002-03 period had disproportionate changes in the shares of the self-employed who were Asian or Hispanic. A shift of this sort between the two periods should cause a sample drawn from the DMI file for 2003 (the SSBF) to understate these rates of minority ownership relative to surveys, such as the SBO, that pick up changes in the population of unincorporated businesses more promptly. Although the 2003 SSBF rates are somewhat lower than the overall self-employment proportions in the 2003 CPS, they are not statistically different from the 2003 CPS proportions of persons self-employed in incorporated businesses.

This analysis provides evidence that minority ownership rates are highest among the smallest firms, the firms most likely to be missing from the DMI file. In addition, the analysis shows differential trends for minority self-employment. Taken together, these findings provide some insight into the dissimilarity of the comparisons between the 1997 SBO and the 1998 SSBF on the one hand, and the 2002 SBO and the 2003 SSBF on the other.

\section{Differences in Coverage}

Both the SSBF and the SBO limit their samples to firms in business as of certain defined periods, but the SSBF changed its population definition between the 1998 and 2003 surveys. The SBO, focused on owner characteristics, restricts its coverage to businesses in operation at any point during the calendar year; the 1997 survey covered businesses in operation during 1997, and the 2002 survey covered businesses in operation during 2002. 
The SSBF, on the other hand, attempts to collect a large amount of financial information about firms that were in business as of December 31 of the survey year. In 2003, an additional in-business constraint was imposed on firms so that account information could be captured as of the date of the interview and balance sheet information could be collected as of the end of the fiscal year (December 31, 2003, for the majority of firms). ${ }^{49}$ Thus, whereas the 1998 SSBF collected information on firms that were in business as of December 31, 1998, the 2003 SSBF collected information on firms that were in business as of December 31, 2003, and at the date of the interview (between June 2004 and January 2005). The 2003 procedure thus effectively imposed a longer longevity constraint on the population of interest.

Because there is a significant amount of "churning" in the business population each year (table B.5), the added longevity constraint implies that fewer firms would be eligible under the 2003 SSBF eligibility rules than under the 1998 SSBF rules. Research indicates that minority-owned businesses are less likely to remain in business than non-minority-owned businesses. ${ }^{50}$ Among the non-minority-owned businesses established in 1997, 72.6 percent were still in business as of 2001. Among Asian-owned businesses, the corresponding proportion was 72.1 percent; among Hispanic-owned businesses, 68.6 percent; and among black-owned businesses, 61.0 percent. Some evidence also indicates that the longer a firm is in business, and the larger a firm is at a given time, the more likely it is to survive over some finite period. ${ }^{51}$

Given (1) the significant churning of businesses, (2) the fact that many minority-owned businesses are quite small, and (3) the higher business closure rates by minorities, we should expect to see fewer minority businesses in the SSBF sample than in the SBO sample even if the sample were drawn from the same list. Furthermore, given that the longevity constraint

49. The additional in-operation restriction was imposed so that respondents could provide information on accounts and financial service providers as of the date of the interview. Although previous interviews asked respondents to provide this information as of the end of the last fiscal year, interviewer reports indicated that many respondents gave account information as of the interview date. To make the reported information consistent across all respondents, the questionnaire was changed to ask about accounts as of the date of the interview.

50. Ying Lowrey (2005), "Dynamics of Minority-Owned Employer Establishments, 1997-2001: An Analysis of Employer Data from the Survey of Minority-Owned Business Establishments," Research Study, U.S. Small Business Administration, Office of Advocacy (February), www.sba.gov/advo/research/chron.html.

51. Joel Popkin and Company (1992), "Business Survival Rates by Age Cohort of Business," Research Study, U.S. Small Business Administration, Office of Advocacy; summary at www.sba.gov/advo/ research/chron.html.
B.5. Number of starts, closures, and bankruptcies of businesses with paid employees, 2000-04 Percent

\begin{tabular}{c|r|r|r|r|c}
\hline Category & 2000 & 2001 & 2002 & 2003 & 2004 \\
\hline Starts ....... & 574,300 & 585,140 & 569,750 & 612,296 & $642,600^{\mathrm{e}}$ \\
Closures .... & 542,831 & 553,291 & 586,890 & 540,658 & $544,300^{\mathrm{e}}$ \\
Bankruptcies . & 35,472 & 40,099 & 38,540 & 35,037 & 34,317 \\
\hline
\end{tabular}

e Estimated.

SOURCE: SBA Office of Advocacy, http://app1.sba.gov/faqs/ faqIndexAll.cfm? areaid $=24$.

applied only to the $2003 \mathrm{SSBF}$, we should expect to observe differences in comparisons of estimates of rates of minority ownership between the 1997 SBO and the 1998 SSBF and between the 2002 SBO and the 2003 SSBF.

\section{Differences in Methodology}

Another area that might have contributed to the observed differences in minority ownership rates between the latest SSBF and SBO can be found in the different ways the two surveys drew their samples. For the 1997 and 2002 SBO, predicted race and ethnicity categories were used as sampling strata, and minorities were oversampled. The 1998 SSBF also oversampled minorities using information collected during the screening interview. The 2003 SSBF did not, however, oversample minorities. The effects of this divergence between the surveys are unclear, but the methodological difference itself is worth noting.

\section{Summary}

In contrast to earlier years, the 2003 SSBF reported lower rates of business ownership by Asians, by blacks, and by Hispanics than did the 2002 SBO. This appendix looked at differences in the universes, coverage, and methodology across the two surveys. Each of these three factors, together with the changing dynamics of the small business population, could be expected to contribute to the differential ownership rates observed between the most recent surveys by the SSBF and SBO.

- The SSBF draws its sample from a list that is likely to be slower than the SBO list in picking up the newest and smallest firms. Between 1997 and 1998, the fluctuations in the self-employment population were relatively stable within racial and ethnic categories. Between 2002 and 2003, however, there were sizable increases in the proportion of selfemployed individuals who were Asian or Hispanic, and the SSBF likely underestimated that recent rise because its source list was slow to record it. 
- The 2003 SSBF extended the time that a firm had to be in business so as to capture certain financial data more accurately. That change would tend, however, to cause fewer minority-owned businesses to qualify for the survey relative to the SBO and to the 1998 SSBF because small and minority-owned firms tend to close more quickly than others.

- Finally, of the four surveys at issue (the two most recent releases of the SBO and the two most recent of the SSBF), the 2003 SSBF was the only one that did not oversample minority-owned businesses, a difference with specific effects that are not known.
Although counterfactuals-such as the percentage of firms that went out of business that are included in the SBO sample or the newly formed firms that are not included in the DMI frame-are not available to definitively identify the cause of the differences, and although all of the factors are likely to have played a role, the differences in the list from which the samples were drawn appear to have been the dominant factor. 